\title{
On the Kummer construction for Kcsc metrics
}

\author{
Claudio Arezzo ${ }^{1,2}$ (D) Alberto Della Vedova ${ }^{3} \cdot$ Riccardo Lena $^{5} \cdot$ Lorenzo Mazzieri $^{4}$
}

Received: 17 January 2018 / Accepted: 5 September 2018

(c) Unione Matematica Italiana 2018

\begin{abstract}
Given a compact constant scalar curvature Kähler orbifold, with nontrivial holomorphic vector fields, whose singularities admit a local ALE Kähler Ricci-flat resolution, we find sufficient conditions on the position of the singular points to ensure the existence of a global constant scalar curvature Kähler desingularization. We also give complete proofs of a number of analytic results which have been used in this context by various authors. A series of explicit examples is discussed.
\end{abstract}

Mathematics Subject Classification 58E11 · 32C17

\section{Introduction}

In this paper we review and extend various existence results for Kähler constant scalar curvature (Kcsc from now on) metrics on compact complex manifolds and orbifolds.

In order to state our results precisely, let us briefly recall that one starts with a Kcsc base $M$ with isolated quotient singularities, hence locally of the form $\mathbb{C}^{m} / \Gamma_{j}$, where $m$ is the complex dimension of $M, j \in J$ parametrizes the set of points we want to desingularize, and $\Gamma_{j}$ is a finite subgroup of $U(m)$ acting freely away from the origin.

In memory of Paolo, friend and teacher.

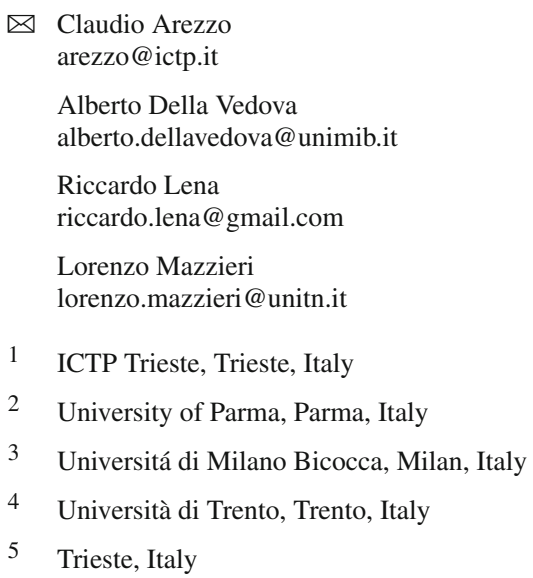


Given such a singular object one would like to remove a small neighborhood of a singular point and replace it with a (scaled down) large piece of a Kähler resolution $\pi:\left(X_{\Gamma}, \eta\right) \rightarrow \mathbb{C}^{m} / \Gamma$ keeping the scalar curvature constant (and close to the starting one). For such a construction to even have a chance to preserve the Kcsc equation it is necessary that $\left(X_{\Gamma}, \eta\right)$ is scalar flat, i.e. it is necessary to assume that $\mathbb{C}^{m} / \Gamma_{j}$ has a scalar flat ALE resolution.

It is well known (see Sect. 2 for a precise description) that such a metric has the following shape,

$$
\eta=i \partial \bar{\partial}\left(\frac{|x|^{2}}{2}+e_{X_{\Gamma}}|x|^{4-2 m}-c_{X_{\Gamma}}|x|^{2-2 m}+\psi_{\eta}(x)\right), \quad \text { with } \quad \psi_{\eta}=\mathcal{O}\left(|x|^{-2 m}\right),
$$

for some real constants $e_{X_{\Gamma}}$ and $c_{X_{\Gamma}}$. In particular the number $e_{X_{\Gamma}}$ is called the ADM mass of the model (see [13] for a comprehensive study of its importance).

Having then fixed a set of singular points $S=\left\{p_{1}, \ldots, p_{n}\right\} \subset M$ each corresponding to a group $\Gamma_{j}$, and denoted by $B_{j, r}:=\left\{z \in \mathbb{C}^{m} / \Gamma_{j}:|z|<r\right\}$, we can define, for all $r>0$ small enough (say $r \in\left(0, r_{0}\right)$ ),

$$
M_{r}:=M \backslash \cup_{j} B_{j, r} .
$$

On the other side, for each $j=1, \ldots, n$, we are given a $m$-dimensional Kähler manifold $\left(X_{\Gamma_{j}}, \eta_{j}\right)$, with one end biholomorphic to a neighborhood of infinity in $\mathbb{C}^{m} / \Gamma_{j}$. Dual to the previous notations on the base manifold, we set $C_{j, R}:=\left\{x \in \mathbb{C}^{m} / \Gamma_{j}:|x|>R\right\}$, the complement of a closed large ball and the complement of an open large ball in $X_{\Gamma_{j}}$ (in the coordinates which parameterize a neighborhood of infinity in $X_{\Gamma_{j}}$ ). We define, for all $R>0$ large enough (say $R>R_{0}$ ),

$$
X_{\Gamma_{j}, R}:=X_{\Gamma_{j}} \backslash C_{j, R}
$$

which corresponds to the manifold $X_{\Gamma_{j}}$ whose end has been truncated. The boundary of $X_{\Gamma_{j}, R}$ is denoted by $\partial C_{j, R}$.

We are now in a position to describe the generalized connected sum construction. Indeed, for all $\varepsilon \in\left(0, r_{0} / R_{0}\right)$, we choose $r_{\varepsilon} \in\left(\varepsilon R_{0}, r_{0}\right)$ and define,

$$
R_{\varepsilon}:=\frac{r_{\varepsilon}}{\varepsilon} .
$$

By construction,

$$
\tilde{M}:=M \sqcup_{p_{1}, \varepsilon} X_{\Gamma_{1}} \sqcup_{p_{2}, \varepsilon} \cdots \sqcup_{p_{n}, \varepsilon} X_{\Gamma_{n}},
$$

is obtained by connecting $M_{r_{\varepsilon}}$ with the truncated ALE spaces $X_{\Gamma_{1, R_{\varepsilon}}}, \ldots, X_{\Gamma_{n, R_{\varepsilon}}}$. The identification of the boundary $\partial B_{j, r_{\varepsilon}}$ in $M_{r_{\varepsilon}}$ with the boundary $\partial C_{j, R_{\varepsilon}}$ of $X_{\Gamma_{j}, R_{\varepsilon}}$ is performed using the change of variables,

$$
\left(z^{1}, \ldots, z^{m}\right)=\varepsilon\left(x^{1}, \ldots, x^{m}\right),
$$

where $\left(z^{1}, \ldots, z^{m}\right)$ are the coordinates in $B_{j, r_{0}}$ and $\left(x^{1}, \ldots, x^{m}\right)$ are the coordinates in $C_{j, R_{0}}$.

It was proved in [4] that if no nontrivial holomorphic vector fields exist on $(M, \omega, g)$ the ALE scalar flat condition on the model is also sufficient to construct a family parametrized by the gluing parameter $\varepsilon$ on the manifold (or orbifold) obtained by this procedure. On the other hand, the known picture for the blow up of smooth points, suggests that the number and position of points should be relevant to achieve the same existence theorem in presence of continuous symmetries. In fact, being the linearized scalar curvature operator $\mathbb{L}_{\omega}$ given by,

$$
\mathbb{L}_{\omega} f=\Delta_{\omega}^{2} f+4\left\langle\rho_{\omega} \mid i \partial \bar{\partial} f\right\rangle
$$


we have to look at the positions of points relative to the elements of $\operatorname{ker}\left(\mathbb{L}_{\omega}\right)=$ $\operatorname{span}_{\mathbb{R}}\left\{\varphi_{0}, \varphi_{1}, \ldots, \varphi_{d}\right\}$, where $\varphi_{0} \equiv 1, d$ is a positive integer and $\varphi_{1}, \ldots, \varphi_{d}$ is a collection of linearly independent functions in $\operatorname{ker}\left(\mathbb{L}_{\omega}\right)$ with zero mean and normalized in such a way that $\left\|\varphi_{i}\right\|_{L^{2}(M)}=1, i=1, \ldots, d$,

As it turns out, the hardest case is when the resolution has zero ADM mass (for example when $\eta$ is actually Ricci flat, which in turn forces the group $\Gamma_{j}$ to be in $S U(m)$ ).

The following is our main result which gives the new conditions on the "symplectic" positions of the singular points for the Kcsc equation to be solvable, thus generalising in the Kcsc context the celebrated Kummer's construction for Calabi-Yau manifolds ([15,17,22,28] for a number of generalisations):

Theorem 1.1 Let $(M, \omega, g)$ be a compact m-dimensional Kcsc orbifold with isolated singularities and constant scalar curvature equal to $s_{\omega}$. Let $\mathbf{p}=\left\{p_{1}, \ldots, p_{N}\right\} \subseteq M$ the set of points with neighborhoods biholomorphic to a ball of $\mathbb{C}^{m} / \Gamma_{j}$ where, for $j=1, \ldots, N$, the $\Gamma_{j}$ 's are nontrivial subgroups of $U(m)$ such that $\mathbb{C}^{m} / \Gamma_{j}$ admits an ALE Ricci-flat resolution $\left(X_{\Gamma_{j}}, h_{j}, \eta_{j}\right)$ with vanishing ADM mass. Let,

$$
\operatorname{ker}\left(\mathbb{L}_{\omega}\right)=\operatorname{span}_{\mathbb{R}}\left\{1, \varphi_{1}, \ldots, \varphi_{d}\right\} .
$$

be the space of Hamiltonian potentials of Killing fields with zeros and assume that $N \geq d$. Suppose moreover that there exists $\mathbf{b} \in\left(\mathbb{R}^{+}\right)^{N}$ such that,

$$
\begin{cases}\sum_{j=1}^{N} b_{p}\left(\Delta_{\omega} \varphi_{j}+s_{\omega} \varphi_{j}\right)(p)=0 & j=1, \ldots, d \\ \left(\left(\Delta_{\omega} \varphi_{j}+s_{\omega} \varphi_{j}\right)(p)\right)_{1 \leq j \leq d, 1 \leq j \leq N} & \text { has rankd. }\end{cases}
$$

Then there exists $\bar{\varepsilon}$ such that for every $\varepsilon \in(0, \bar{\varepsilon})$ the orbifold,

$$
\tilde{M}:=M \sqcup_{p_{1}, \varepsilon} X_{\Gamma_{1}} \sqcup_{p_{2}, \varepsilon} \cdots \sqcup_{p_{N}, \varepsilon} X_{\Gamma_{N}},
$$

has a Kcsc metric in the class,

$$
\pi^{*}[\omega]+\sum_{j=1}^{N} \varepsilon^{2 m} \tilde{b}_{j}^{2 m}\left[\tilde{\eta}_{j}\right] \quad \text { with } i_{j}^{*}\left[\tilde{\eta}_{j}\right]=\left[\eta_{j}\right],
$$

where $\pi$ is the canonical surjection of $\tilde{M}$ onto $M$ and $\mathfrak{i}_{j}$ the natural embedding of $X_{\Gamma_{j}, R_{\varepsilon}}$ into $\tilde{M}$. Moreover,

$$
\left|\tilde{b}_{j}^{2 m}-\frac{\left|\Gamma_{j}\right| b_{j}}{2(m-1)}\right| \leq C_{\varepsilon}^{\gamma} \quad \text { for some } \quad \gamma>0
$$

where $\left|\Gamma_{j}\right|$ denotes the order of the group.

A version (with a non explicit balancing formula) of the previous result was announced without proof in [3] (Theorem 1.2). In this paper we provide the complete proof and make explicit the right balancing condition.

Whether, given $\Gamma$ in $S U(m)$, a Ricci flat Kähler resolution exists is by itself an important problem in different areas of mathematics and we will not digress on it here. It suffices to recall the reader that Ricci flat models do exist for any subgroup of $S U(m)$ with $m=2$, thanks to the work of Kronheimer, while in higher dimensions one needs to assume the existence of a Kähler crepant resolution and then apply deep results by Joyce [15], Goto [12], Van Coevering [29] and Conlon-Hein [8]. In particular $m=3$ works fine again for any $\Gamma$ in $S U(3)$. 
On the other hand, and contrary to the authors' first expectations, Hein-LeBrun [13] have proved the existence of many new examples of scalar flat with vanishing ADM mass but not Ricci flat.

The proof we present in this paper follows the path of previous result of this type [3-5]. In this respect the main difficulty comes from the fact that, contrary to all these previous known cases, part of the obstruction, comes from the non linear analysis.

An alternative proof would rely instead of the existence result for extremal metrics proved in [3]. Mixing this fact (whose analytic difficulties are undoubtedly much easier than the present's one) with the classical observation of Calabi [7] that an extremal metric in a class with vanishing Futaki invariant is in fact Kcsc, leads to the delicate problem of how to compute this invariant for resolutions of isolated quotient singularities. This is accomplished in [1] and this will also allow to generalize the previous Theorem to the general case of scalar-flat resolutions and to prove also the necessity of the balancing condition.

The proof we present here certainly requires a deeper and finer understanding of the PDE point of view, but has the advantage, at least under these stronger assumptions, to give a more refined information on the Kcsc metric produced. In fact as we will see in Sect. 3, each singular point gives us the freedom of the choice of two parameters ( $b$ and $c$ ). Indeed for each choice of these parameters the metrics $\omega_{\mathbf{0}, \mathbf{b}, \mathbf{c}, \mathbf{h}, \mathbf{k}}$ can be actually glued to the metric $\eta$ (of course not solving any particular PDE), but it is easy to check that the resulting Kähler class depends only on $b$ and not on $c$. As we will show in the final part of the proof of Theorem 1.1, the nonlinear part of the Kcsc equation will require a uniquely determined choice of $c$ as a function of $b\left(c=s_{\omega} b\right)$, in accordance with the well known uniqueness property of Kcsc metrics in a given Kähler class. At the end of the proof one will then know explicitly how to write down the first two exploding terms in the asymptotic expansion of the Kähler potential of the final Kcsc metric, a result out of reach if one takes the alternative route of passing through extremal metrics and Futaki invariants.

We can then look for new examples of full or partial desingularizations of Kcsc orbifolds. Of course it will be very hard on a general orbifold to compute $\Delta_{\omega} \varphi_{j}$. On the other hand, assuming for example that $M$ is Einstein and using,

$$
\Delta_{\omega} \varphi_{j}=-\frac{s_{\omega}}{m} \varphi_{j},
$$

the balancing condition requires only the knowledge of the value of the $\varphi_{j}$ at the singular points. Moreover these values are easily computed for example in toric setting by the well known relationship between the evaluation of the potentials $\varphi_{j}$ and the image point via the moment map.

With these classical observations one can then look for toric Kähler-Einstein orbifolds with isolated quotient singularities to test to which of them our results can be applied. In complex dimension two things are pretty simple and in fact two such examples are,

- $\left(\mathbb{P}^{1} \times \mathbb{P}^{1}, \pi_{1}^{*} \omega_{F S}+\pi_{2}^{*} \omega_{F S}\right)$ with $\mathbb{Z}_{2}$ acting by,

$$
\left(\left[x_{0}: x_{1}\right],\left[y_{0}: y_{1}\right]\right) \longrightarrow\left(\left[x_{0}:-x_{1}\right],\left[y_{0}:-y_{1}\right]\right) .
$$

This orbifold is isomorphic to the intersection of two singular, quadrics in $\mathbb{P}^{4}$.

$$
\left\{z_{0} z_{3}-z_{4}^{2}=0\right\} \cap\left\{z_{1} z_{2}-z_{4}^{2}=0\right\} .
$$

- $\left(\mathbb{P}^{2}, \omega_{F S}\right)$ with $\mathbb{Z}_{3}$ acting by,

$$
\left[z_{0}: z_{1}: z_{2}\right] \longrightarrow\left[x_{0}: \zeta_{3} x_{1}: \zeta_{3}^{2} x_{2}\right] \quad \zeta_{3} \neq 1, \zeta_{3}^{3}=1 .
$$


This orbifold is isomorphic to the singular cubic surface in, $\mathbb{P}^{3}$

$$
\left\{z_{0} z_{1} z_{2}-z_{3}^{3}=0\right\}
$$

In both cases we will show in Sect. 7 that our results provide a full Kcsc (clearly not Kähler-Einstein) desingularization (in the first case applied to 4 singular $S U$ (2) points, while $3 S U$ (2) points in the second). It is worth noting that both these orbifolds are also limits of smooth Kähler- Einstein surfaces. This can be seen in various ways: either applying Tian's resolution of the Calabi Conjecture [27] or by [2] in the first case, or by Odaka-Spotti-Sun [21].

Working out higher dimensional examples turned out to be much more challenging than we expected. Even making use of the beautiful database of Toric Fano Threefolds run by Brown and Kasprzyk ([10], see also [16]) and their amazing help in implementing a complete search of Einstein ones with isolated singularities, we could only extract orbifolds where only a partial Kcsc resolution is possible. We will describe two such examples in Sect. 7.

It is interesting to observe that in the list of toric Fano KE 3-folds it appears a very intriguing example which does not satisfy any balancing condition, neither the one in Theorem 1.1 nor those of [3]. It seems a very intriguing question whether this example be actually desingularized by Kcsc manifolds in some way. Beyond this example, it is worth mentioning, among the possible extensions of the present technique and the possible directions of research, the desingularization of Kcsc manifolds with non isolated singularities, in the spirit of the generalized connected sum constructions performed in [18-20].

Structure of the paper In Sect. 2 we collect some known facts and we prove a crucial refinement (Proposition 2.3) of results of Joyce, Tian-Yau and others on the asymptotics of a Kähler Ricci flat metric on a crepant resolution.

In Sect. 3 we collect all results needed at the linear level on the linearized scalar curvature operator on the base orbifold. In particular we construct global functions in the kernel of the linearized operator with prescribed blow up behaviour near the singularities (see Proposition 3.3).

Section 4 contains the (weighted) linear analysis on a scalar flat Kähler resolution of an isolated singularity. These results are significantly different from what was known, in that our problem forces us to use weights in a different, more delicate, range.

In Sect. 5 the existence of truncated Kcsc metrics on the base and on the models is proved in Propositions 5.1 and 5.2.

Section 6 contains the analytic proof of Theorem 1.1 by proving the mentioned Cauchydata matching property of the truncated metrics previously produced.

Section 7 gives a complete description of the above mentioned examples.

\section{Notations and preliminaries}

\subsection{Eigenfunctions and eigenvalues of $\Delta_{\mathbb{S} 2 m-1}$}

In order to fix some notation which will be used throughout the paper, we agree that $\mathbb{S}^{2 m-1}$ is the unit sphere of real dimension $2 m-1$, equipped with the standard round metric inherited from $\left(\mathbb{C}^{m}, g_{\text {eucl }}\right)$. We will denote by $\left\{\phi_{k}\right\}_{k \in \mathbb{N}}$ a complete orthonormal system of the Hilbert space $L^{2}\left(\mathbb{S}^{2 m-1}\right)$, given by eigeinfunctions of the Laplace-Beltrami operator $\Delta_{\mathbb{S}^{2 m-1}}$, so that, for every $k \in \mathbb{N}$, 


$$
\Delta_{\mathbb{S}^{2 m-1}} \phi_{k}=\lambda_{k} \phi_{k},
$$

and $\left\{\lambda_{k}\right\}_{k \in \mathbb{N}}$ are the eigenvalues of $\Delta_{\mathbb{S}^{2 m-1}}$ counted with multiplicity. We will also indicate by $\Phi_{j}$ the generic element of the $j$-th eigenspace of $\Delta_{\mathbb{S}^{2 m-1}}$, so that, for every $j \in \mathbb{N}$,

$$
\Delta_{\mathbb{S}^{2 m-1}} \Phi_{j}=\Lambda_{j} \Phi_{j}
$$

and $\left\{\Lambda_{j}\right\}_{j \in \mathbb{N}}$ are the eigenvalue of $\Delta_{\mathbb{S}}^{2 m-1}$ counted without multiplicity. In particular, we have that $\Lambda_{j}=-j(2 m-2+j)$, for every $j \in \mathbb{N}$. If $\Gamma \triangleleft U(m)$ is a finite subgroup of the unitary group acting on $\mathbb{C}^{m}$ having the origin as its only fixed point, we denote by $\left\{\Lambda_{j}^{\Gamma}\right\}_{j \in \mathbb{N}}$ the eigenvalues counted without multiplicity of the operator $\Delta_{\mathbb{S}^{2 m-1}}$ restricted to the $\Gamma$-invariant functions. For future convenience we introduce the following notation, given $f \in L^{2}\left(\mathbb{S}^{2 m-1}\right)$ we denote with $f^{(k)}$ the $L^{2}\left(\mathbb{S}^{2 m-1}\right)$-projection of $f$ on the $\Lambda_{k}$-eigenspace of $\Delta_{\mathbb{S}^{2 m-1}}$ and,

$$
f^{(\dagger)}:=f-f^{(0)} .
$$

\subsection{The scalar curvature equation}

We let $(M, g, \omega)$ be a Kähler orbifold with complex dimension equal to $m$, where $g$ is the Kähler metric and $\omega$ is the Kähler form. Notice that we allow the Riemannian orbifold $(M, g)$ to be incomplete, since in the following we will be eventually led to consider punctured orbifolds. We denote by $s_{\omega}$ the scalar curvature of the Kähler metric $g$ and by $\rho_{\omega}$ its Ricci form. In the following it will be useful to consider cohomologous deformations of the Kähler form $\omega$. Hence, for a smooth real function $f \in C^{\infty}(M)$ such that $\omega+i \partial \bar{\partial} f>0$, we set,

$$
\omega_{f}=\omega+i \partial \bar{\partial} f,
$$

and we will refer to $f$ as the deformation potential. Since we want to understand the behavior of the scalar curvature under deformations of this type, it is convenient to consider the following differential operator,

$$
\mathbf{S}_{\omega}(\cdot): C^{\infty}(M) \longrightarrow C^{\infty}(M), \quad f \longmapsto \mathbf{S}_{\omega}(f):=s_{\omega+i \partial \bar{\partial} f},
$$

which associate to a deformation potential $f$ the scalar curvature of the corresponding metric. We then have,

$$
\mathbf{S}_{\omega}(f)=s_{\omega}-\frac{1}{2} \mathbb{L}_{\omega} f+\frac{1}{2} \mathbb{N}_{\omega}(f),
$$

where the linearized scalar curvature operator $\mathbb{L}_{\omega}$ is given by,

$$
\mathbb{L}_{\omega} f=\Delta_{\omega}^{2} f+4\left\langle\rho_{\omega} \mid i \partial \bar{\partial} f\right\rangle .
$$

Once we introduce the bilinear operator o acting on tensors in $\left(T M^{*}\right)^{(1,0)} \otimes\left(T M^{*}\right)^{(0,1)}$ as,

$$
(T \circ U)_{i \bar{l}}:=T_{i \bar{\jmath}} g^{k \bar{\jmath}} U_{k \bar{l}} \quad T, U \in\left(T M^{*}\right)^{(1,0)} \otimes\left(T M^{*}\right)^{(0,1)},
$$

the nonlinear remainder $\mathbb{N}_{\omega}$ takes the form,

$$
\begin{aligned}
\mathbb{N}_{\omega}(f)= & 8 \operatorname{tr}_{\omega}\left(i \partial \bar{\partial} f \circ i \partial \bar{\partial} f \circ \rho_{\omega}\right)-8 \operatorname{tr}_{\omega}\left(i \partial \bar{\partial} f \circ i \partial \bar{\partial} \Delta_{\omega} f\right) \\
& +4 \Delta_{\omega} \operatorname{tr}_{\omega}(i \partial \bar{\partial} f \circ i \partial \bar{\partial} f)+2 \mathbb{R}_{\omega}(f),
\end{aligned}
$$

with $\mathbb{R}_{\omega}(f)$ the collections of all higher order terms. 


\subsection{The Kähler potential of a Kcsc orbifold}

We let $(M, g, \omega)$ be a compact constant scalar curvature Kähler orbifold without boundary with complex dimension equal to $m$. Unless otherwise stated the singularities are assumed to be isolated.

Combining the local $\partial \bar{\partial}$-lemma with the equations of the previous section, we now recall a more precise well-known description of the local structure of the Kähler potential of a Kcsc metric.

Proposition 2.1 Let $(M, g, \omega)$ be a Kähler orbifold. Then, given any point $p \in M$, there exists a holomorphic coordinate chart $\left(U, z^{1}, \ldots, z^{m}\right)$ centered at $p$ such that the Kähler form can be written as,

$$
\omega=i \partial \bar{\partial}\left(\frac{|z|^{2}}{2}+\psi_{\omega}\right), \quad \text { with } \quad \psi_{\omega}=\mathcal{O}\left(|z|^{4}\right) .
$$

If in addition the scalar curvature $s_{g}$ of the metric $g$ is constant, then $\psi_{g}$ is a real analytic function on $U$, and one can write,

$$
\psi_{\omega}(z, \bar{z})=\sum_{k=0}^{+\infty} \Psi_{4+k}(z, \bar{z}),
$$

where, for every $k \in \mathbb{N}$, the component $\Psi_{4+k}$ is a real homogeneous polynomial in the variables $z$ and $\bar{z}$ of degree $4+k$. In particular, we have that $\Psi_{4}$ and $\Psi_{5}$ satisfy the equations,

$$
\begin{aligned}
& \Delta^{2} \Psi_{4}=-2 s_{\omega}, \\
& \Delta^{2} \Psi_{5}=0,
\end{aligned}
$$

where $\Delta$ is the Euclidean Laplace operator of $\mathbb{C}^{m}$. Finally, the polynomial $\Psi_{4}$ can be written as,

$$
\Psi_{4}(z, \bar{z})=\left(-\frac{s_{\omega}}{16 m(m+1)}+\Phi_{2}+\Phi_{4}\right)|z|^{4},
$$

where $\Phi_{2}$ and $\Phi_{4}$ are functions in the second and fourth eigenspace of $\Delta_{\mathbb{S}^{2 m-1}}$, respectively.

\subsection{The Kähler potential of a scalar flat ALE Kähler resolution}

We start by recalling the concept of Asymptotically Locally Euclidean (ALE for short) Kähler resolution of an isolated quotient singularitiy. We let $\Gamma \triangleleft U(\mathrm{~m})$ be a finite subgroup of the unitary group acting freely away from the origin. and we say that a complete noncompact Kähler manifold $\left(X_{\Gamma}, h, \eta\right)$ of complex dimension $m$, where $h$ is the Kähler metric and $\eta$ is the Kähler form, is an ALE Kähler manifold with group $\Gamma$ if there exist a positive radius $R>0$ and a quotient map $\pi: X_{\Gamma} \rightarrow \mathbb{C}^{m} / \Gamma$, such that,

$$
\pi: X_{\Gamma} \backslash \pi^{-1}\left(B_{R}\right) \longrightarrow\left(\mathbb{C}^{m} \backslash B_{R}\right) / \Gamma,
$$

is a biholomorphism and in standard Euclidean coordinates the metric $\pi_{*} h$ satisfies the expansion,

$$
\left|\frac{\partial^{\alpha}}{\partial x^{\alpha}}\left(\left(\pi_{*} h\right)_{i \bar{j}}-\frac{1}{2} \delta_{i \bar{j}}\right)\right|=\mathcal{O}\left(|x|^{-\tau-|\alpha|}\right),
$$

for some $\tau>0$ and every multindex $\alpha \in \mathbb{N}^{m}$. 
Remark 2.1 The reader must be aware of the fact that the above definition gives only a special class of Kähler ALE manifolds. In particular we are identifying the complex structure outside a compact subset with the standard one, while in general it could be only asymptotic to it and in fact the complex structure could not even admit holomorphic coordinates at infinity as shown for example by Honda [14] also in the scalar flat case.

Remark 2.2 In the following, we will make as systematic use of $\pi$ as an identification and, consequently, we will make no difference between $h$ and $\pi_{*} h$ as well as between $\eta$ and $\pi_{*} \eta$.

Remark 2.3 It is a simple exercise to prove that if $\Gamma$ is nontrivial, then there are no $\Gamma$ invariant linear functions on $\mathbb{C}^{m}$, and thus, with the notations introduced in Sect. 2.1, we have that $\Lambda_{1}^{\Gamma}>\Lambda_{1}$. This will be repeatedly used in our arguments in Proposition 2.3 and Proposition 4.2.

We are now ready to present a result which describe the asymptotic behaviour of the Kähler potential of a scalar flat ALE Kähler metric. This can be though as the analogous of Proposition 2.1. We omit the proof because in the spirit it is very similar to the one of the aforementioned proposition and the details can be found in [4].

Proposition 2.2 Let $\left(X_{\Gamma}, h, \eta\right)$ be a scalar flat ALE Kähler resolution of an isolated quotient singularitiy and let $\pi: X_{\Gamma} \rightarrow \mathbb{C}^{m} / \Gamma$ be the quotient map. Then for $R>0$ large enough, we have that on $X_{\Gamma} \backslash \pi^{-1}\left(B_{R}\right)$ the Kähler form can be written as,

$$
\eta=i \partial \bar{\partial}\left(\frac{|x|^{2}}{2}+e_{X_{\Gamma}}|x|^{4-2 m}-c_{X_{\Gamma}}|x|^{2-2 m}+\psi_{\eta}(x)\right), \text { with } \psi_{\eta}=\mathcal{O}\left(|x|^{-2 m}\right),
$$

for some real constants $e_{X_{\Gamma}}$ and $c_{X_{\Gamma}}$. Moreover, the radial component $\psi_{\eta}^{(0)}$ in the Fourier decomposition of $\psi_{\eta}$ is such that,

$$
\psi_{\eta}^{(0)}(|x|)=\mathcal{O}\left(|x|^{6-4 m}\right) .
$$

In the case where the ALE Kähler metric is Ricci-flat it is possible to obtain sharper estimates for the deviation of the Kähler potential from the Euclidean one, which we believe to be of independent interest. This is far form being obvious and in fact it is an important result of Joyce [15, Theorem 8.2.3 pag 175]. With the following proposition we now give an improvement of Joyce's result which will turn out to be crucial in the rest of the paper.

Proposition 2.3 Let $\left(X_{\Gamma}, h, \eta\right)$ be as in Proposition 2.2. Moreover let $\Gamma \triangleleft U(m)$ be nontrivial and $e(\Gamma)=0$. Then for $R>0$ large enough, we have that on $X_{\Gamma} \backslash \pi^{-1}\left(B_{R}\right)$ the Kähler form can be written as

$$
\eta=i \partial \bar{\partial}\left(\frac{|x|^{2}}{2}-c_{X_{\Gamma}}|x|^{2-2 m}+\psi_{\eta}(x)\right), \text { with } \psi_{\eta}=\mathcal{O}\left(|x|^{-2 m}\right),
$$

for some positive real constant $c_{X_{\Gamma}}>0$. Moreover, the radial component $\psi_{\eta}^{(0)}$ in the Fourier decomposition of $\psi_{\eta}$ is such that,

$$
\psi_{\eta}^{(0)}(|x|)=\mathcal{O}\left(|x|^{2-4 m}\right) .
$$

Proof By [15, Theorem 8.2.3], we have that on $X_{\Gamma} \backslash \pi^{-1}\left(B_{R}\right)$ the Kähler form $\eta$ can be written as,

$$
\eta=i \partial \bar{\partial}\left(\frac{|x|^{2}}{2}-c_{X_{\Gamma}}|x|^{2-2 m}+\psi_{\eta}(x)\right) \text { with } \psi_{\eta}(x)=\mathcal{O}\left(|x|^{2-2 m-\gamma}\right),
$$


for some $\gamma \in(0,1)$. Since $\left(X_{\Gamma}, h\right)$ is scalar flat, arguing as in Proposition 2.1, we deduce that $\psi_{\eta}$ is a real analytic function. To obtain the desired estimates on the decay of $\psi_{\eta}$, we are going to make use of the equation $\mathbf{S}_{\text {eucl }}\left(\psi_{\eta}-c_{X_{\Gamma}}|x|^{2-2 m}\right)=0$. By means of identity (2.1), (2.2) and (2.3), this can be rephrased in terms of $\psi_{\eta}$ as follows,

$$
\begin{aligned}
\Delta^{2} \psi_{\eta}= & 8 \operatorname{tr}\left(i \partial \bar{\partial}\left(\psi_{\eta}-c_{X_{\Gamma}}|x|^{2-2 m}\right) \circ i \partial \bar{\partial} \Delta \psi_{\eta}\right) \\
& +4 \Delta \operatorname{tr}\left(i \partial \bar{\partial}\left(\psi_{\eta}-c_{X_{\Gamma}}|x|^{2-2 m}\right) \circ i \partial \bar{\partial}\left(\psi_{\eta}-c_{X_{\Gamma}}|x|^{2-2 m}\right)\right) \\
& +2 \mathbb{R}_{\text {eucl }}\left(\psi_{\eta}-c_{X_{\Gamma}}|x|^{2-2 m}\right),
\end{aligned}
$$

where, in writing the first summand on the right hand side, we have used the fact that $\Delta|x|^{2-2 m}=0$. Since $\psi_{\eta}=\mathcal{O}\left(|x|^{2-2 m-\gamma}\right)$, for some $\gamma \in(0,1)$, it is straightforward to see that all of the terms on the right hand side can be estimated as $\mathcal{O}\left(|x|^{-2-4 m-\gamma}\right)$, with the only exception of the purely radial term,

$$
\Delta \operatorname{tr}\left(\left(i \partial \bar{\partial}|x|^{2-2 m}\right) \circ\left(i \partial \bar{\partial}|x|^{2-2 m}\right)\right)=\mathcal{O}\left(|x|^{-2-4 m}\right) .
$$

For sake of convenience, we set now the right hand side of the above equation equal to $F / 2$, so that,

$$
\Delta^{2} \psi_{\eta}=F
$$

It is now convenient to expand both $\psi_{\eta}$ and $F$ in Fourier series as,

$$
\psi_{\eta}(x)=\sum_{k=0}^{+\infty} \psi_{\eta}^{(k)}(|x|) \phi_{k}(x /|x|) \text { and } F(x)=\sum_{k=0}^{+\infty} F^{(k)}(|x|) \phi_{k}(x /|x|),
$$

where the functions $\left\{\phi_{k}\right\}_{k \in \mathbb{N}}$, are the eigenfunctions of the spherical laplacian $\Delta_{\mathbb{S}^{2 m-1}}$ on $\mathbb{S}^{2 m-1}$, counted with multplicity. Since $\phi_{0} \equiv\left|\mathbb{S}^{2 m-1}\right|^{-1 / 2}$, we will refer to $\psi_{\eta}^{(0)}$ and $F^{(0)}$ as the radial part of $\psi_{\eta}$ and $F$, respectively. We also notice that in the forthcoming discussion it will be important to select among the eigenfunctions $\phi_{k}$ 's, only the ones which are $\Gamma$-invariant, in order to respect the quotient structure. So far, we have seen that $F^{(0)}=\mathcal{O}\left(|x|^{-2-4 m}\right)$ and $F^{(k)}=\mathcal{O}\left(|x|^{-2-4 m-\gamma}\right)$, for $k \geq 1$. On the other hand, using the linear ODE satisfied by the components $\psi_{\eta}^{(k)}$, it is not hard to see that their general expression is given by,

$$
\psi_{\eta}^{(k)}(|x|)=a_{k}|x|^{4-2 m-\alpha(k)}+b_{k}|x|^{2-2 m-\alpha(k)}+c_{k}|x|^{\alpha(k)}+d_{k}|x|^{\alpha(k)+2}+\tilde{\psi}_{\eta}^{(k)}(|x|),
$$

where, in view of the behavior of the $F^{(k)}$ 's, the functions $\tilde{\psi}_{\eta}^{(k)}$ are such that,

$$
\tilde{\psi}_{\eta}^{(0)}=\mathcal{O}\left(|x|^{2-4 m}\right) \quad \text { and } \quad \tilde{\psi}_{\eta}^{(k)}=\mathcal{O}\left(|x|^{2-4 m-\gamma}\right), \quad \text { for } k \geq 1,
$$

and the integers $\alpha(k)$ 's are such that $\alpha(k)=h$ if and only if $\phi_{k}$ belongs to the $h$-th eigenspace. Since the cited Joyce's result implies that $\psi_{\eta}^{(k)}=\mathcal{O}\left(|x|^{2-2 m-\gamma}\right)$, it is easy to deduce that $c_{k}=0=d_{k}$, for every $k \in \mathbb{N}$. Moreover, we have that $a_{0}=0=b_{0}$ and thus $\psi_{\eta}^{(0)}=$ $\mathcal{O}\left(|x|^{2-4 m}\right)$, as wanted. The same kind of considerations imply that the components $\psi_{\eta}^{(k)}$, s satisfy the desired estimates for every $k \geq 2 m+1$, that is for every $k$ such that $\alpha(k) \geq 2$. For $1 \leq k \leq 2 m$, we have that $a_{k}=0$, but a priori nothing can be said about the $b_{k}$ 's and thus at a first glance, one has that,

$$
\psi_{\eta}^{(k)}(|x|)=b_{k}|x|^{1-2 m}+\tilde{\psi}_{\eta}^{(k)}(|x|), \quad \text { for } 1 \leq k \leq 2 m .
$$

As it has been pointed out in Remark 2.3, there are no $\Gamma$-invariant eigenfunctions for $\Delta_{\mathbb{S} 2 m-1}$ in the first eigenspace. This means that the components $\psi_{\eta}^{k}$, s, with $1 \leq k \leq 2 m$ do not appear in the Fourier expansion of $\psi_{\eta}$ and hence $\psi_{\eta}(x)=\mathcal{O}\left(|x|^{-2 m}\right)$. 
We conclude this section with the following, probably well known observation, which will turn out to be very useful in our proof. The authors are not aware of a reference of it so we include its proof for the reader's convenience.

Proposition 2.4 If $(X, \eta)$ is Ricci flat, we have,

$$
d \mu_{\eta}=\pi^{*} d \mu_{0}
$$

and for $R>0$

$$
\operatorname{Vol}_{\eta}\left(X_{\Gamma, R}\right)=\frac{\left|\mathbb{S}^{2 m-1}\right|}{2 m|\Gamma|} R^{2 m}
$$

Proof Let $\pi_{\Gamma}: \mathbb{C}^{m} \rightarrow \mathbb{C}^{m} / \Gamma$ the canonical holomorphic quotient map, since,

$$
\rho_{\eta}=0,
$$

on $\left(\mathbb{C}^{m} \backslash B_{R}\right) / \Gamma$ we have,

$$
i \partial \bar{\partial}\left[\log \left(\operatorname{det}\left(\left(\pi_{\Gamma}\right)^{*}\left(\pi^{-1}\right)^{*} \eta\right)\right)\right]=0 .
$$

We want to prove that on $\mathbb{C}^{m} \backslash\{0\}$,

$$
\operatorname{det}\left(\left(\pi_{\Gamma}\right)^{*}\left(\pi^{-1}\right)^{*} \eta\right) \equiv \frac{1}{2^{m}} .
$$

By Proposition 2.3 we have on $\mathbb{C}^{m} \backslash B_{R}$,

$$
\left(\pi_{\Gamma}\right)^{*}\left(\pi^{-1}\right)^{*} \eta_{i \bar{j}}=\frac{\delta_{i \bar{j}}}{2}-c(\Gamma) \partial_{i} \bar{\partial}_{j}|x|^{2-2 m}+\mathcal{O}\left(|x|^{-2 m}\right),
$$

that implies immediately,

$$
\log \left(\operatorname{det}\left(\left(\pi_{\Gamma}\right)^{*}\left(\pi^{-1}\right)^{*} \eta\right)\right)=-m \log (2)+\mathcal{O}\left(|x|^{-2-2 m}\right) .
$$

On $\mathbb{C}^{m} \backslash B_{R}$ we have,

$$
i \partial \bar{\partial} \log \left(\operatorname{det}\left(\left(\pi_{\Gamma}\right)^{*}\left(\pi^{-1}\right)^{*} \eta\right)\right)=-i d\left(\partial \log \left(\operatorname{det}\left(\left(\pi_{\Gamma}\right)^{*}\left(\pi^{-1}\right)^{*} \eta\right)\right)\right),
$$

so,

$$
\partial \log \left(\operatorname{det}\left(\left(\pi_{\Gamma}\right)^{*}\left(\pi^{-1}\right)^{*} \eta\right)\right) \in H^{1}\left(\mathbb{C}^{m} \backslash B_{R}, \mathbb{C}\right),
$$

but $H^{1}\left(\mathbb{C}^{m} \backslash B_{R}, \mathbb{C}\right)=0$ and there exists $h_{1} \in C^{1}\left(\mathbb{C}^{m} \backslash B_{R}, \mathbb{C}\right)$ such that,

$$
\partial \log \left(\operatorname{det}\left(\left(\pi_{\Gamma}\right)^{*}\left(\pi^{-1}\right)^{*} \eta\right)\right)=d h_{1}=\partial h_{1}+\bar{\partial} h_{1} \quad \bar{\partial} h_{1}=0 .
$$

Analogously, there is $h_{2} \in C^{1}\left(\mathbb{C}^{m} \backslash B_{R}, \mathbb{C}\right)$ such that,

$$
\bar{\partial}\left[\log \left(\operatorname{det}\left(\left(\pi_{\Gamma}\right)^{*}\left(\pi^{-1}\right)^{*} \eta\right)\right)-h_{1}\right]=d h_{2}=\partial h_{2}+\bar{\partial} h_{2} \quad \partial h_{2}=0 .
$$

It is now clear that,

$$
d\left[\log \left(\operatorname{det}\left(\left(\pi_{\Gamma}\right)^{*}\left(\pi^{-1}\right)^{*} \eta\right)\right)-h_{1}-h_{2}\right]=0 .
$$

We conclude that on $\mathbb{C}^{m} \backslash B_{R}$,

$$
\log \left(\operatorname{det}\left(\left(\pi_{\Gamma}\right)^{*}\left(\pi^{-1}\right)^{*} \eta\right)\right)=h_{1}+h_{2}+K \quad K \in \mathbb{R} \quad \Im \mathfrak{m} h_{2}=-\Im \mathfrak{m} h_{1},
$$


moreover $h_{1}, \overline{h_{2}}$ are holomorphic on $\mathbb{C}^{m} \backslash B_{R}$ and by Hartogs extension theorem they are extendable to functions $H_{1}, H_{2}$ holomorphic on $\mathbb{C}^{m}$. Since $H_{1}, H_{2}$ are holomorphic, their real and imaginary parts are harmonic with respect to the euclidean metric on $\mathbb{C}^{m}$ and by assumptions on $\eta$ we have on $\mathbb{C}^{m} \backslash B_{R}$,

$$
\mathfrak{R e} H_{1}+\mathfrak{I m} H_{2}+K=-m \log (2)+\mathcal{O}\left(|x|^{-2-2 m}\right) .
$$

Since $\mathfrak{R e} H_{1}+\mathfrak{I m} H_{2}+K$ is harmonic and bounded, Liouville theorem implies it is constant, so that,

$$
\operatorname{det}\left(\left(\pi_{\Gamma}\right)^{*}\left(\pi^{-1}\right)^{*} \eta\right)=\frac{1}{2^{m}}
$$

We can now see that,

$$
\frac{1}{m !}\left(\pi_{\Gamma}\right)^{*}\left[\left(\pi^{-1}\right)^{*} \eta\right]^{\wedge m}=d \mu_{0}
$$

and then,

$$
\operatorname{Vol}_{\eta}\left(X_{\Gamma, R}\right)=\int_{B_{R} / \Gamma \backslash\{0\}} d \mu_{\left(\pi^{-1}\right)^{*} \eta}=\frac{\left|\mathbb{S}^{2 m-1}\right|}{2 m|\Gamma|} R^{2 m},
$$

so also the third statement is proved.

\section{Linear analysis on a Kcsc orbifold}

In this section we develop the linear analysis for the operator $\mathbb{L}_{\omega}$ and we do it in full generality even if, in this work, we will use only some particular cases of this theory. We distinguish between two sets of points: $\left\{p_{1}, \ldots, p_{N}\right\}$ with neighborhoods biholomorphic to a ball of $\mathbb{C}^{m} / \Gamma_{j}$ with $\Gamma_{j}$ nontrivial such that $\mathbb{C}^{m} / \Gamma_{j}$ admits an ALE Kahler scalar-flat resolution $\left(X_{\Gamma_{j}}, h, \eta_{j}\right)$ with $e_{X_{\Gamma_{j}}}=0$ and the set (possibly empty) $\left\{q_{1}, \ldots, q_{K}\right\}$ whose points have neighborhoods biholomorphic to a ball of $\mathbb{C}^{m} / \Gamma_{N+l}$ such that $\mathbb{C}^{m} / \Gamma_{N+l}$ admits a scalar flat ALE resolution $\left(Y_{\Gamma_{N+l}}, k_{l}, \theta_{l}\right)$ with $e_{X_{\Gamma_{N+l}}} \neq 0$. To simplify the notation we set,

$$
\mathbf{p}:=\left\{p_{1}, \ldots, p_{N}\right\}, \quad \mathbf{q}:=\left\{q_{1}, \ldots, q_{K}\right\}, \quad \text { and } \quad M_{\mathbf{p}, \mathbf{q}}:=M \backslash(\mathbf{p} \cup \mathbf{q}) .
$$

CAVEAT We agree that, if $\mathbf{q}=\emptyset$, then $M_{\mathbf{p}}:=M_{\mathbf{p}, \emptyset .}$. When this case occurs and whenever an object, that could be a function or a tensor, has indices relative to elements of $\mathbf{q}$ we set these indices to 0 .

\subsection{The bounded kernel of $\mathbb{L}_{\omega}$}

As usual we let $(M, g, \omega)$ be a compact Kcsc orbifold with isolated singularities and we assume that the kernel of the linearized scalar curvature operator $\mathbb{L}_{\omega}$ defined in (2.2) is nontrivial, in the sense that it contains also nonconstant functions. By the standard Fredholm theory for self-adjoint elliptic operators, we have that such a kernel is always finite dimensional. Throughout the paper we will assume that it is $(d+1)$-dimensional and we will set,

$$
\operatorname{ker}\left(\mathbb{L}_{\omega}\right)=\operatorname{span}_{\mathbb{R}}\left\{\varphi_{0}, \varphi_{1}, \ldots, \varphi_{d}\right\},
$$

where $\varphi_{0} \equiv 1, d$ is a positive integer and $\varphi_{1}, \ldots, \varphi_{d}$ is a collection of linearly independent functions in $\operatorname{ker}\left(\mathbb{L}_{\omega}\right)$ with zero mean and normalized in such a way that $\left\|\varphi_{i}\right\|_{L^{2}(M)}=1$, 
$i=1, \ldots, d$, for sake of simplicity. We recall the following classical characterization of $\operatorname{ker}\left(\mathbb{L}_{\omega}\right)$.

Proposition 3.1 Let $(M, g, \omega)$ be a compact constant scalar curvature Kähler orbifold with isolated singularities. Then, the subspace of $\operatorname{ker}\left(\mathbb{L}_{\omega}\right)$ given by the elements with zero mean is in one to one correspondence with the space of holomorphic vector fields which vanish somewhere in $M$.

The aim of this section is to study the solvability of the linear problem,

$$
\mathbb{L}_{\omega} u=f,
$$

on the complement of the singular points in $M$. In order to do that, we introduce some notation as well as an appropriate functional setting. We consider geodesics balls $B_{r_{0}}\left(p_{j}\right), B_{r_{0}}\left(q_{l}\right)$ of radius $r_{0}>0$, with Kähler normal coordinates centered at the points $p_{j}$ 's and $q_{l}$ 's and we set,

$$
M_{r_{0}}:=M \backslash\left(\bigcup_{j=1}^{N} B_{r_{0}}\left(p_{j}\right) \cup \bigcup_{l=1}^{K} B_{r_{0}}\left(q_{l}\right)\right) .
$$

For $\delta \in \mathbb{R}$ and $\alpha \in(0,1)$, we define the weighted Hölder space $C_{\delta}^{k, \alpha}\left(M_{\mathbf{p}, \mathbf{q}}\right)$ as the set of functions $f \in C_{l o c}^{k, \alpha}\left(M_{\mathbf{p}, \mathbf{q}}\right)$ such that the norm,

$$
\begin{aligned}
\|f\|_{C_{\delta}^{k, \alpha}\left(M_{\mathbf{p}, \mathbf{q}}\right)}:= & \|f\|_{C^{k, \alpha}\left(M_{r_{0}}\right)}+\sup _{0<r \leq r_{0}} r^{-\delta} \sum_{j=1}^{N}\left\|\left.f(r \cdot)\right|_{B_{r_{0}}\left(p_{j}\right)}\right\|_{C^{k, \alpha}\left(B_{2} \backslash B_{1}\right)} \\
& +\sup _{0<r \leq r_{0}} r^{-\delta} \sum_{l=1}^{K}\left\|\left.f(r \cdot)\right|_{B_{r_{0}}\left(q_{l}\right)}\right\|_{C^{k, \alpha}\left(B_{2} \backslash B_{1}\right)},
\end{aligned}
$$

is finite. We observe that the typical function $f \in C_{\delta}^{4, \alpha}\left(M_{\mathbf{p}, \mathbf{q}}\right)$ beheaves like,

$$
f(\cdot)=\mathcal{O}\left(d_{\omega}\left(p_{j}, \cdot\right)^{\delta}\right), \quad \text { on } B_{r_{0}}\left(p_{j}\right) \text { and } f(\cdot)=\mathcal{O}\left(d_{\omega}\left(q_{j}, \cdot\right)^{\delta}\right), \quad \text { on } B_{r_{0}}\left(q_{j}\right) \text {, }
$$

where $d_{\omega}$ is the Riemannian distance induced by the Kahler metric $\omega$.

We are now in the position to solve Eq. (3.2) in the case where the datum $f$ is orthogonal to $\operatorname{ker}\left(\mathbb{L}_{\omega}\right)$. By this we mean that, looking at $f$ as a distribution, we have,

$$
\left\langle f \mid \varphi_{i}\right\rangle_{\mathscr{D}^{\prime} \times \mathscr{D}}=0,
$$

for every $i=0, \ldots, d$, where we denoted by $\langle\cdot \mid \cdots\rangle_{\mathscr{D}^{\prime} \times \mathscr{D}}$ the distributional pairing and the functions $\varphi_{i}$ 's are as in (3.1). It is worth pointing out that since the functions in $\operatorname{ker}\left(\mathbb{L}_{\omega}\right)$ are smooth, everything makes sense.

To solve Eq. (3.2) we need to ensure the Fredholmness of the operator $\mathbb{L}_{\omega}$ on the functional spaces we have chosen. The Fredholm property depends heavily on the choice of weights, indeed the operator $\mathbb{L}_{\omega}$ is Fredholm if and only if the weight is not an indicial root (for definition of indicial roots we refer to [5]) at any of the points $p_{j}$ 's or $q_{l}$ 's. Since in normal coordinates on a punctured ball, the principal part of our operator $\mathbb{L}_{\omega}$ is 'asymptotic' to the Euclidean Laplacian $\Delta$, then the set of indicial roots of $\mathbb{L}_{\omega}$ at the center of the ball coincides with the set of indicial roots of $\Delta$ at 0 . We recall that the set of indicial roots of $\Delta$ at 0 is given by $\mathbb{Z} \backslash\{5-2 m, \ldots,-1\}$ for $m \geq 3$ and $\mathbb{Z}$ for $m=2$. 
By the analysis in [4], we recover the following result, which provides the existence of solutions in Sobolev spaces for the linearized equation together with a priori estimates in suitable weighted Hölder spaces.

Theorem 3.1 For every $f \in L^{p}(M), p>1$, satisfying the orthogonality condition (3.3), there exists a unique solution $u \in W^{4, p}(M)$ to,

$$
\mathbb{L}_{\omega} u=f,
$$

which satisfy the condition (3.3). Moreover, the following estimates hold true.

- If $m \geq 3$ and in addition $f \in C_{\delta-4}^{0, \alpha}\left(M_{\mathbf{p}, \mathbf{q}}\right)$ with $\delta \in(4-2 m, 0)$, then the solution $u$ belongs to $C_{\delta}^{4, \alpha}\left(M_{\mathbf{p}, \mathbf{q}}\right)$ and satisfy the estimates,

$$
\|u\|_{C_{\delta}^{4, \alpha}\left(M_{\mathbf{p}, \mathbf{q}}\right)} \leq C\|f\|_{C_{\delta-4}^{0, \alpha}\left(M_{\mathbf{p}, \mathbf{q}}\right)},
$$

for some positive constant $C>0$.

- If $m=2$ and in addition $f \in C_{\delta-4}^{0, \alpha}\left(M_{\mathbf{p}, \mathbf{q}}\right)$ with $\delta \in(0,1)$, then the solution $u$ belongs to $C_{\text {loc }}^{4, \alpha}\left(M_{\mathbf{p}, \mathbf{q}}\right)$ and satisfy the following estimates,

$$
\begin{aligned}
& \left\|u-\sum_{j=1}^{N} u\left(p_{j}\right) \chi_{p_{j}}-\sum_{l=1}^{K} u\left(q_{l}\right) \chi_{q_{l}}\right\|_{C_{\delta}^{4, \alpha}\left(M_{\mathbf{p}, \mathbf{q}}\right)}+\sum_{j=1}^{N}\left|u\left(p_{j}\right)\right|+\sum_{l=1}^{K}\left|u\left(q_{l}\right)\right| \\
& \leq C\|f\|_{C_{\delta-4}^{0, \alpha}\left(M_{\mathbf{p}, \mathbf{q}}\right)},
\end{aligned}
$$

where $C>0$ is a positive constant and the functions $\chi_{p_{1}}, \ldots, \chi_{p_{N}}$ and $\chi_{q_{1}}, \ldots, \chi_{q_{K}}$ are smooth cutoff functions supported on small balls centered at the points $p_{1}, \ldots, p_{N}$ and $q_{1}, \ldots, q_{K}$, respectively and identically equal to 1 in a neighborhood of these points.

In order to drop the orthogonality assumption (3.3) in Theorem 3.1 and tackle the general case, we first need to investigate the behaviour of the fundamental solutions of the operator $\mathbb{L}_{\omega}$. This will be done in the following section.

\subsection{Multi-poles fundamental solutions of $\mathbb{L}_{\omega}$}

The aim of this section is twofold. On one hand, we want to produce the tools for solving Eq. (3.2) on $M_{\mathbf{p}, \mathbf{q}}$, when $f$ is not necessarily orthogonal to $\operatorname{ker}\left(\mathbb{L}_{\omega}\right)$. On the other hand, we are going to determine under which global conditions on $\operatorname{ker}\left(\mathbb{L}_{\omega}\right)$ we can produce a function, which near the singularities behaves like the principal non euclidean part of the Kähler potential of the corresponding ALE resolution. In concrete, building on Propositions 2.3 and 2.2, we aim to establish the existence of a function, which blows up like $|z|^{2-2 m}$ near the $p_{j}$ 's and like $|z|^{4-2 m}$ near the $q_{l}$ 's. Such a function will then be added to the original Kähler potential of the base orbifold in order to make it closer to the one of the resolution. At the same time, for obvious reasons, it is important to guarantee that this new Kähler potential will produce on $M_{\mathbf{p}, \mathbf{q}}$ the smallest possible deviation from the original scalar curvature, at least at the linear level. Thinking of $g$ as a perturbation of the flat metric at small scale, we have that $\mathbb{L}_{\omega}$ can be thought of as a perturbation of $\Delta^{2}$. Since $|z|^{2-2 m}$ and $|z|^{4-2 m}$ satisfy equations of the form,

$$
\Delta^{2}\left(A|z|^{2-2 m}+B|z|^{4-2 m}\right)=C \Delta \delta_{0}+D \delta_{0},
$$


where $\delta_{0}$ is the Dirac distribution centered at the origin and $A, B, C$ and $D$ are suitable constants, we are led to study these type of equations on $M$ for the operator $\mathbb{L}_{\omega}$.

The following easily follows from standard arguments in functional analysis.

Proposition 3.2 Let $(M, g, \omega)$ be compact Kcsc orbifold of complex dimension $m$ and let $\operatorname{ker}\left(\mathbb{L}_{\omega}\right)=\operatorname{span}\left\{\varphi_{0}, \varphi_{1}, \ldots, \varphi_{d}\right\}$, as in (3.1). Let $\left(f_{0}, \ldots, f_{d}\right)$ be a vector in $\mathbb{R}^{d+1}$. Assume that the following linear balancing condition holds

$$
\begin{gathered}
f_{i}+\sum_{l=1}^{K} a_{l} \varphi_{i}\left(q_{l}\right)+\sum_{j=1}^{N} b_{j}\left(\Delta \varphi_{i}\right)\left(p_{j}\right)+\sum_{j=1}^{N} c_{j} \varphi_{i}\left(p_{j}\right)=0, \quad i=1, \ldots, d, \\
f_{0} \operatorname{Vol}_{\omega}(M)+\sum_{l=1}^{K} a_{l}+\sum_{j=1}^{N} c_{j}=v \operatorname{Vol}_{\omega}(M),
\end{gathered}
$$

for some choice of the coefficients $v, \mathbf{a}=\left(a_{1}, \ldots, a_{K}\right), \mathbf{b}=\left(b_{1}, \ldots, b_{N}\right)$ and $\mathbf{c}=$ $\left(c_{1}, \ldots, c_{N}\right)$. Then, there exist a distributional solution $U \in \mathscr{D}^{\prime}(M)$ to the equation,

$$
\mathbb{L}_{\omega}[U]+v=\sum_{i=0}^{d} f_{i} \varphi_{i}+\sum_{l=1}^{K} a_{l} \delta_{q_{l}}+\sum_{j=1}^{N} b_{j} \Delta \delta_{p_{j}}+\sum_{j=1}^{N} c_{j} \delta_{p_{j}}, \quad \text { in } M .
$$

Remark 3.1 When $f_{i}=0$, for $i=0, \ldots, d$, we only impose the balancing condition (3.6), which specializes to,

$$
\sum_{l=1}^{K} a_{l} \varphi_{i}\left(q_{l}\right)+\sum_{j=1}^{N} b_{j}\left(\Delta \varphi_{i}\right)\left(p_{j}\right)+\sum_{j=1}^{N} c_{j} \varphi_{i}\left(p_{j}\right)=0,
$$

and we obtain a real number $v_{\mathbf{a}, \mathbf{c}}$, defined by the relation,

$$
\sum_{l=1}^{K} a_{l}+\sum_{j=1}^{N} c_{j}=v_{\mathbf{a}, \mathbf{c}} \operatorname{Vol}_{\omega}(M)
$$

and a distribution $\mathbf{G}_{\mathbf{a}, \mathbf{b}, \mathbf{c}} \in \mathscr{D}^{\prime}(M)$, which satisfies the equation,

$$
\mathbb{L}_{\omega}\left[\mathbf{G}_{\mathbf{a}, \mathbf{b}, \mathbf{c}}\right]+v_{\mathbf{a}, \mathbf{c}}=\sum_{l=1}^{K} a_{l} \delta_{q_{l}}+\sum_{j=1}^{N} b_{j} \Delta \delta_{p_{j}}+\sum_{j=1}^{N} c_{j} \delta_{p_{j}}, \quad \text { in } M .
$$

We will refer to $\mathbf{G}_{\mathbf{a}, \mathbf{b}, \mathbf{c}}$ as a multi-poles fundamental solution of $\mathbb{L}_{\omega}$.

The following two lemmata and the subsequent proposition (3.3) will give us a precise description of the behavior of a multi-poles fundamental solution $\mathbf{G}_{\mathbf{a}, \mathbf{b}, \mathbf{c}}$ of $\mathbb{L}_{\omega}$ around the singular points. The same considerations obviously apply to a distributional solution $U$ of the Eq. (3.7). The first observation in this direction can be found in [5] and we report it here for sake of completeness.

Lemma 3.2 Let $(M, g, \omega)$ be a Kcsc orbifold of complex dimension $m \geq 2$ and let $M_{q}=$ $M \backslash\{q\}$, with $q \in M$. Then, the following holds true.

- If $m \geq 3$, there exists a function $G_{\Delta \Delta}(q, \cdot) \in \mathcal{C}_{4-2 m}^{4, \alpha}\left(M_{q}\right) \cap \mathcal{C}_{\text {loc }}^{\infty}\left(M_{q}\right)$, orthogonal to $\operatorname{ker}\left(\mathbb{L}_{\omega}\right)$ inthe sense of (3.3), such that,

$$
\mathbb{L}_{\omega}\left[G_{\Delta \Delta}(q, \cdot)\right]+\frac{2(m-1)\left|\mathbb{S}^{2 m-1}\right|}{|\Gamma|}\left[4(m-2) \delta_{q}\right] \in \mathcal{C}^{0, \alpha}(M),
$$


where $|\Gamma|$ is the order of the orbifold group at $q$. Moreover, if $z$ are holomorphic coordinates centered at $q$, it holds the expansion,

$$
G_{\Delta \Delta}(q, z)=|z|^{4-2 m}+\mathcal{O}\left(|z|^{6-2 m}\right) .
$$

- If $m=2$, there exists a function $G_{\Delta \Delta}(q, \cdot) \in \mathcal{C}_{\text {loc }}^{\infty}\left(M_{q}\right)$, orthogonal to $\operatorname{ker}\left(\mathbb{L}_{\omega}\right)$ inthe sense of (3.3), such that,

$$
\mathbb{L}_{\omega}\left[G_{\Delta \Delta}(q, \cdot)\right]-\frac{4\left|\mathbb{S}^{3}\right|}{|\Gamma|} \delta_{q} \in \mathcal{C}^{0, \alpha}(M),
$$

where $|\Gamma|$ is the order of the orbifold group at $q$. Moreover, if $z$ are holomorphic coordinates centered at $q$, it holds the expansion,

$$
G_{\Delta \Delta}(q, \cdot)=\log (|z|)+C_{q}+\mathcal{O}\left(|z|^{2}\right),
$$

for some constant $C_{q} \in \mathbb{R}$.

Before stating the next lemma, it is worth pointing out that $G_{\Delta \Delta}(q, \cdot)$ has the same rate of blow up as the Green function of the bi-Laplacian operator $\Delta^{2}$. Since we want to produce a local approximation of the multi-poles fundamental solution $\mathbf{G}_{\mathbf{a}, \mathbf{b}, \mathbf{c}}$, we also need a profile whose blow up rate around the singular points is the same as the one of the Green function of the Laplace operator. This will be responsible for the $\Delta \delta_{p}$ 's terms.

Lemma 3.3 Let $(M, g, \omega)$ be a Kcsc orbifold of complex dimension $m \geq 2$ and let $M_{p}=$ $M \backslash\{p\}$, with $p \in M$. Then, the following holds true.

- If $m \geq 3$, there exists a function $G_{\Delta}(p, \cdot) \in \mathcal{C}_{2-2 m}^{4, \alpha}\left(M_{p}\right) \cap \mathcal{C}_{\text {loc }}^{\infty}\left(M_{p}\right)$, orthogonal to $\operatorname{ker}\left(\mathbb{L}_{\omega}\right)$ inthe sense of (3.3), such that,

$$
\mathbb{L}_{\omega}\left[G_{\Delta}(p, \cdot)\right]-\frac{2(m-1)\left|\mathbb{S}^{2 m-1}\right|}{|\Gamma|}\left[\Delta \delta_{p}+\frac{s_{\omega}\left(m^{2}-m+2\right)}{m(m+1)} \delta_{p}\right] \in \mathcal{C}^{0, \alpha}(M),
$$

where $|\Gamma|$ is the cardinality of the orbifold group at $p$ and $s_{\omega}$ is the constant scalar curvature of the orbifold. Moreover, if $z$ are holomorphic coordinates centered at $p$, it holds the expansion,

$$
G_{\Delta}(p, \cdot)=|z|^{2-2 m}+|z|^{4-2 m}\left(\Phi_{2}+\Phi_{4}\right)+|z|^{5-2 m} \sum_{j=0}^{2} \Phi_{2 j+1}+\mathcal{O}\left(|z|^{6-2 m}\right),
$$

for suitable smooth $\Gamma$-invariant functions $\Phi_{j}$ 's defined on $\mathbb{S}^{2 m-1}$ and belonging to the $j$-th eigenspace of the operator $\Delta_{\mathbb{S}^{2 m-1}}$.

- If $m=2$, there exists a function $G_{\Delta}(p, \cdot) \in \mathcal{C}_{-2}^{4, \alpha}\left(M_{p}\right) \cap \mathcal{C}_{\text {loc }}^{\infty}\left(M_{p}\right)$, orthogonal to $\operatorname{ker}\left(\mathbb{L}_{\omega}\right)$ inthe sense of (3.3), such that,

$$
\mathbb{L}_{\omega}\left[G_{\Delta}(p, \cdot)\right]-\frac{\left|\mathbb{S}^{3}\right|}{|\Gamma|} \Delta \delta_{p}-\frac{s_{\omega} 2\left|\mathbb{S}^{3}\right|}{3|\Gamma|} \delta_{p} \in \mathcal{C}^{0, \alpha}(M),
$$

where $|\Gamma|$ is the cardinality of the orbifold group at $p$ and $s_{\omega}$ is the constant scalar curvature of the orbifold. Moreover, if $z$ are holomorphic coordinates centered at $p$, it holds the expansion

$$
G_{\Delta}(p, \cdot)=|z|^{-2}+\log (|z|)\left(\Phi_{2}+\Phi_{4}\right)+C_{p}+|z| \sum_{h=0}^{2} \Phi_{2 h+1}+\mathcal{O}\left(|z|^{2}\right),
$$


for some constant $C_{p} \in \mathbb{R}$, some $H \in \mathbb{N}$ and suitable smooth $\Gamma$-invariant functions $\Phi_{h}$ 's defined on $\mathbb{S}^{3}$ and belonging to the h-th eigenspace of the operator $\Delta_{\mathbb{S}^{3}}$.

Proof We focus on the case $m \geq 3$ and since the computations for the case $m=2$ are very similar, we leave them to the reader. To prove the existence of $G_{\Delta}(p, \cdot)$, we fix a coordinate chart centered at $p$ and we consider the Green function for the Euclidean Laplacian $|z|^{2-2 m}$. In the spirit of Proposition 2.1, we compute,

$$
\begin{aligned}
\mathbb{L}_{\omega}\left[|z|^{2-2 m}\right]= & \left(\mathbb{L}_{\omega}-\Delta^{2}\right)\left[|z|^{2-2 m}\right] \\
= & -4 \operatorname{tr}\left(i \partial \bar{\partial}|z|^{2-2 m} \circ i \partial \bar{\partial} \Delta \psi_{\omega}\right)-4 \operatorname{tr}\left(i \partial \bar{\partial} \psi_{\omega} \circ i \partial \bar{\partial} \Delta|z|^{2-2 m}\right) \\
& -4 \Delta \operatorname{tr}\left(i \partial \bar{\partial} \psi_{\omega} \circ i \partial \bar{\partial}|z|^{2-2 m}\right)+\mathcal{O}\left(|z|^{2-2 m}\right) \\
= & -\frac{m}{4|z|^{2 m}} \Delta^{2} \Psi_{4}+\frac{m(m+1)}{|z|^{2 m+2}} \Delta \Psi_{4}-\frac{m}{4} \Delta\left(\frac{\Delta \Psi_{4}}{|z|^{2 m}}\right) \\
& +4 m(m+1) \Delta \operatorname{tr}\left(\frac{\Psi_{4}}{|z|^{2 m+2}}\right)+\mathcal{O}\left(|z|^{2-2 m}\right),
\end{aligned}
$$

where we used the explicit form of $\Psi_{4}$,

$$
\Psi_{4}(z, \bar{z})=-\frac{1}{4} \sum_{i, j, k, l=1}^{m} R_{i \bar{j} k \bar{l}} z^{i} \overline{z^{j}} z^{k} \overline{z^{l}},
$$

and the complex form of the euclidean laplace operator,

$$
\Delta=4 \sum_{i=1}^{m} \frac{\partial^{2}}{\partial z^{i} \partial \overline{z^{i}}}
$$

Expanding the real analytic function $\psi_{\omega}$ as $\psi_{\omega}=|z|^{4}\left(\Phi_{0}+\Phi_{2}+\Phi_{4}\right)+|z|^{5}\left(\Phi_{1}+\Phi_{3}+\right.$ $\left.\Phi_{5}\right)+\mathcal{O}\left(\left|z^{6}\right|\right)$, where, for $h=0,1,2$, the $\Phi_{2 h}$ 's and the $\Phi_{2 h+1}$ 's are suitable $\Gamma$-invariant functions in the $h$-th eigenspace of $\Delta_{\mathbb{S} 2 m-1}$, we obtain,

$$
\mathbb{L}_{\omega}\left[|z|^{2-2 m}\right]=|z|^{-2 m} \sum_{h=0}^{2} c_{2 h} \Phi_{2 h}+|z|^{1-2 m} \sum_{h=0}^{2} c_{2 h+1} \Phi_{2 h+1}+\mathcal{O}\left(|z|^{2-2 m}\right),
$$

where $c_{0}, \ldots, c_{5}$ are suitable constants. It is a straightforward but remarkable consequence of formula (2.4), the fact that $c_{0}=0$. It is then possible to introduce the corrections,

$$
V_{4}=|z|^{4-2 m}\left(C_{2} \Phi_{2}+C_{4} \Phi_{4}\right) \text { and } V_{5}=|z|^{5-2 m} \sum_{h=0}^{2} C_{2 h+1} \Phi_{2 h+1},
$$

where the coefficients $C_{1}, \ldots, C_{5}$ are so chosen that,

$$
\Delta^{2}\left[V_{4}+V_{5}\right]=|z|^{-2 m}\left(c_{2} \Phi_{2}+c_{4} \Phi_{4}\right)+|z|^{1-2 m} \sum_{h=0}^{2} c_{2 h+1} \Phi_{2 h+1} .
$$

This implies in turn that $\mathbb{L}_{\omega}\left[|z|^{2-2 m}-V_{4}-V_{5}\right]=\mathcal{O}\left(|z|^{2-2 m}\right)$. Using the fact that in normal coordinates centered at $p$ the Euclidean bi-Laplacian operator $\Delta^{2}$ yields a good approximation of $\mathbb{L}_{\omega}$, it is not hard to construct a function $W \in C_{6-2 m}^{4, \alpha}\left(B_{r_{0}}^{*}\right)$ on a sufficiently small punctured ball $B_{r_{0}}^{*}$ centered at $p$, such that,

$$
\mathbb{L}_{\omega}\left[|z|^{2-2 m}-V_{4}-V_{5}-W\right] \in C^{0, \alpha}\left(B_{r_{0}}^{*}\right) .
$$


By means of a smooth cut-off function $\chi$, compactly supported in $B_{r_{0}}$ and identically equal to 1 in $B_{r_{0} / 2}$, we obtain a globally defined function in $L^{1}(M)$, namely,

$$
U_{p}=\chi\left(|z|^{2-2 m}-|z|^{4-2 m}\left(C_{2} \Phi_{2}+C_{4} \Phi_{4}\right)-|z|^{5-2 m} \sum_{h=0}^{2} C_{2 h+1} \Phi_{2 h+1}-W\right)
$$

In order to guarantee the orthogonality condition (3.3), we set,

$$
G_{\Delta}(p, \cdot)=U_{p}(\cdot)-\frac{1}{\operatorname{Vol}_{\omega}(M)} \int_{M} U_{p} d \mu_{\omega}-\sum_{i=1}^{d} \varphi_{i}(\cdot) \int_{M} U_{p} \varphi_{i} d \mu_{\omega},
$$

and we claim that $\mathbb{L}_{\omega}\left[G_{\Delta}(p, \cdot)\right]$ satisfies the desired distributional identity. To see this, we set $M_{\varepsilon}=M \backslash B_{\varepsilon}$, where $B_{\varepsilon}$ is a ball of radius $\varepsilon$ centered at $p$, and we integrate $\mathbb{L}_{\omega}\left[G_{\Delta}(p, \cdot)\right]=$ $\mathbb{L}_{\omega}\left[U_{p}\right]$ against a test function $\phi \in C^{\infty}(M)$. Setting,

$$
\rho_{\omega}^{0}=\rho_{\omega}-\frac{s_{\omega}}{2 m} \omega
$$

and using formula (2.2), it is convenient to write,

$$
\mathbb{L}_{\omega}\left[U_{p}\right]=\Delta_{\omega}^{2} U_{p}+\frac{s_{\omega}}{m} \Delta_{\omega} U_{p}+4\left\langle\rho_{\omega}^{0} \mid i \partial \bar{\partial} U_{p}\right\rangle,
$$

so that we have,

$$
\int_{M_{\varepsilon}} \phi \mathbb{L}_{\omega}\left[U_{p}\right] d \mu_{\omega}=\int_{M_{\varepsilon}} \phi\left(\Delta_{\omega}^{2}+\frac{s_{\omega}}{m} \Delta_{\omega}\right)\left[U_{p}\right] d \mu_{\omega}+4 \int_{M_{\varepsilon}} \phi\left\langle\rho_{\omega}^{0} \mid i \partial \bar{\partial} U_{p}\right\rangle d \mu_{\omega} .
$$

We first integrate by parts the first summand on the right hand side and we take the limit for $\varepsilon \rightarrow 0$, obtaining,

$$
\begin{aligned}
\lim _{\varepsilon \rightarrow 0} & \int_{M_{\varepsilon}} \phi\left(\Delta_{\omega}^{2}+\frac{s_{\omega}}{m} \Delta_{\omega}\right)\left[U_{p}\right] d \mu_{\omega} \\
= & \int_{M} U_{p}\left(\Delta_{\omega}^{2}+\frac{s_{\omega}}{m} \Delta_{\omega}\right)[\phi] d \mu_{\omega}+\lim _{\varepsilon \rightarrow 0} \int_{\partial M_{\varepsilon}} \phi \partial_{\nu}\left(\Delta_{\omega} U_{p}\right) d \sigma_{\omega} \\
& +\lim _{\varepsilon \rightarrow 0} \int_{\partial M_{\varepsilon}}\left(\Delta_{\omega} \phi\right) \partial_{\nu} U_{p} d \sigma_{\omega}+\frac{s_{\omega}}{m} \lim _{\varepsilon \rightarrow 0} \int_{\partial M_{\varepsilon}} \phi \partial_{\nu} U_{p} d \sigma_{\omega}
\end{aligned}
$$

where $d \sigma_{\omega}$ is the restriction of the measure $d \mu_{\omega}$ to $\partial M_{\varepsilon}$ and $v$ is the exterior unit normal to $\partial M_{\varepsilon}$. Combining the definition of $U_{p}$ with the standard development of the area element, it is easy to deduce that,

$$
\begin{gathered}
\lim _{\varepsilon \rightarrow 0} \int_{\partial M_{\varepsilon}}\left(\Delta_{\omega} \phi\right) \partial_{\nu} U_{p} d \sigma_{\omega}+\frac{s_{\omega}}{m} \lim _{\varepsilon \rightarrow 0} \int_{\partial M_{\varepsilon}} \phi \partial_{\nu} U_{p} d \sigma_{\omega} \\
=\frac{2(m-1)\left|\mathbb{S}^{2 m-1}\right|}{|\Gamma|}\left[\Delta_{\omega} \phi(p)+\frac{s_{\omega}}{m} \phi(p)\right] .
\end{gathered}
$$

To treat the last boundary term, we use Proposition 2.1 and we compute,

$$
\partial_{\nu}\left(\Delta_{\omega} U_{p}\right)=|z|^{1-2 m}\left(\frac{2 s_{\omega}(m-1)^{3}}{m(m+1)}+K_{2} \Phi_{2}+K_{4} \Phi_{4}\right)+\mathcal{O}\left(|z|^{2-2 m}\right),
$$

for suitable constants $K_{2}$ and $K_{4}$. Hence, we get,

$$
\lim _{\varepsilon \rightarrow 0} \int_{\partial M_{\varepsilon}} \phi \partial_{\nu}\left(\Delta_{\omega} U_{p}\right) d \sigma_{\omega}=\frac{2(m-1)\left|\mathbb{S}^{2 m-1}\right|}{|\Gamma|}\left[\frac{s_{\omega}(m-1)^{2}}{m(m+1)} \phi(p)\right] .
$$


In conclusion we have that,

$$
\begin{aligned}
\left\langle\left(\Delta_{\omega}^{2}+\frac{s_{\omega}}{m} \Delta_{\omega}\right)\left[U_{p}\right] \mid \phi\right\rangle_{\mathscr{D}^{\prime} \times \mathscr{D}}= & \int_{M} U_{p}\left(\Delta_{\omega}^{2}+\frac{s_{\omega}}{m} \Delta_{\omega}\right)[\phi] d \mu_{\omega} \\
& +\frac{2(m-1)\left|\mathbb{S}^{2 m-1}\right|}{|\Gamma|}\left[\Delta_{\omega} \phi(p)+\frac{s_{\omega}\left(m^{2}-m+2\right)}{m(m+1)} \phi(p)\right] .
\end{aligned}
$$

We now pass to consider the term contanining $\rho_{\omega}^{0}$. An integration by parts gives,

$$
\begin{aligned}
\lim _{\varepsilon \rightarrow 0} \int_{M_{\varepsilon}} \phi\left\langle\rho_{\omega}^{0} \mid i \partial \bar{\partial} U_{p}\right\rangle d \mu_{\omega}= & \int_{M} U_{p}\left\langle\rho_{\omega}^{0} \mid i \partial \bar{\partial} \phi\right\rangle d \mu_{\omega} \\
& \left.\left.+\lim _{\varepsilon \rightarrow 0} \int_{\partial M_{\varepsilon}} \phi X\left(U_{p}\right)\right\lrcorner d \mu_{\omega}+\lim _{\varepsilon \rightarrow 0} \int_{\partial M_{\varepsilon}} U_{p} \overline{X(\phi)}\right\lrcorner d \mu_{\omega},
\end{aligned}
$$

where, for a given function $u \in C^{1}\left(M_{p}\right)$, the vector field $X(u)$ is defined as $X(u)=$ $\left(\rho_{\omega}^{0}\left(\partial^{\sharp} u, \cdot\right)\right)^{\sharp}$. It is easy to check that second boundary term vanishes in the limit. We claim that the same is true for the first boundary term. To prove this, we recall the expansions,

$$
\begin{aligned}
\left(\rho_{\omega}^{0}\right)_{i \bar{j}} & =\left(\lambda_{i}(p)-\frac{s_{\omega}}{2 m}\right) \delta_{i \bar{j}}+\mathcal{O}(|z|), \\
\partial^{\sharp} U_{p} & =\sum_{i=1}^{m}\left((1-m)|z|^{-2 m} z^{i}+\mathcal{O}\left(|z|^{2-2 m}\right)\right) \frac{\partial}{\partial z^{i}} \\
d \mu_{\omega} & =\left(1+\mathcal{O}\left(|z|^{2}\right)\right) d \mu_{0},
\end{aligned}
$$

where the $\lambda_{i}$ 's are the eigenvalues of the matrix $\left(\rho_{\omega}^{0}\right)_{i \bar{j}}$ and $d \mu_{0}$ is the Euclideam volume form. This implies,

$$
\left.\left.X\left(U_{p}\right)\right\lrcorner d \mu_{\omega}=(1-m) \sum_{i=1}^{m}\left(\lambda_{i}(p)-\frac{s_{\omega}}{2 m}\right) z^{i} \frac{\partial}{\partial z^{i}}\right\lrcorner d \mu_{0}+\mathcal{O}(|z|) .
$$

On the other hand, by the symmetry of $d \mu_{0}$, it is easy to deduce that,

$$
\left.\left.\int_{\partial M_{\varepsilon}} z^{1} \frac{\partial}{\partial z^{1}}\right\lrcorner d \mu_{0}=\cdots=\int_{\partial M_{\varepsilon}} z^{m} \frac{\partial}{\partial z^{m}}\right\lrcorner d \mu_{0} .
$$

The claim is now a straightforward consequence. In synthesis, we have obtained,

$$
\begin{aligned}
\left\langle\mathbb{L}_{\omega}\left[U_{p}\right] \mid \phi\right\rangle_{\mathscr{D}^{\prime} \times \mathscr{D}}= & \int_{M} U_{p} \mathbb{L}_{\omega}[\phi] d \mu_{\omega}+\frac{2(m-1)\left|\mathbb{S}^{2 m-1}\right|}{|\Gamma|} \\
& \times\left[\Delta_{\omega} \phi(p)+\frac{s_{\omega}\left(m^{2}-m+2\right)}{m(m+1)} \phi(p)\right]
\end{aligned}
$$

and the lemma is proven.

Having at hand the above lemmata, we are now in the position to describe the local structure around the singular points of the multi-poles fundamental solutions $\mathbf{G}_{\mathbf{a}, \mathbf{b}, \mathbf{c}}$ constructed in Remark 3.1 through Proposition 3.2. For $m \geq 3$, it is sufficient to apply the operator $\mathbb{L}_{\omega}$ to 
the expression,

$$
\begin{aligned}
& \mathbf{G}_{\mathbf{a}, \mathbf{b}, \mathbf{c}}+\sum_{l=1}^{K} \frac{a_{l}}{4(m-2)}\left[\frac{\left|\Gamma_{N+l}\right|}{2(m-1)\left|\mathbb{S}^{2 m-1}\right|} G_{\Delta \Delta}\left(q_{l}, \cdot\right)\right] \\
& +\sum_{j=1}^{N}\left(\frac{c_{j}}{4(m-2)}-\frac{s_{\omega}\left(m^{2}-m+2\right) b_{j}}{(m-2) m(m+1)}\right)\left[\frac{\left|\Gamma_{j}\right|}{2(m-1)\left|\mathbb{S}^{2 m-1}\right|} G_{\Delta \Delta}\left(p_{j}, \cdot\right)\right] \\
& -\sum_{j=1}^{N} b_{j}\left[\frac{\left|\Gamma_{j}\right|}{2(m-1)\left|\mathbb{S}^{2 m-1}\right|} G_{\Delta}\left(p_{j}, \cdot\right)\right]
\end{aligned}
$$

to get a function in $C^{0, \alpha}(M)$. For $m=2$, one can obtain the same conclusion, applying the operator $\mathbb{L}_{\omega}$ to the expression,

$$
\begin{aligned}
& \mathbf{G}_{\mathbf{a}, \mathbf{b}, \mathbf{c}}-\sum_{l=1}^{K} \frac{a_{l}}{4}\left[\frac{\left|\Gamma_{N+l}\right|}{\left|\mathbb{S}^{3}\right|} G_{\Delta \Delta}\left(q_{l}, \cdot\right)\right] \\
& -\sum_{j=1}^{N}\left(\frac{c_{j}}{4}-\frac{s_{\omega} b_{j}}{6}\right)\left[\frac{\left|\Gamma_{j}\right|}{\left|\mathbb{S}^{3}\right|} G_{\Delta \Delta}\left(p_{j}, \cdot\right)\right] \\
& -\sum_{j=1}^{N} b_{j}\left[\frac{\left|\Gamma_{j}\right|}{2\left|\mathbb{S}^{3}\right|} G_{\Delta}\left(p_{j}, \cdot\right)\right] .
\end{aligned}
$$

Combining the previous observations with the standard elliptic regularity theory, we obtain the following proposition.

Proposition 3.3 Let $(M, g, \omega)$ be a compact Kcsc orbifold of complex dimension $m \geq 2$, let $\operatorname{Ker}\left(\mathbb{L}_{\omega}\right)=\operatorname{span}\left\{\varphi_{0}, \varphi_{1}, \ldots, \varphi_{d}\right\}$, as in (3.1) and let $\mathbf{G}_{\mathbf{a}, \mathbf{b}, \mathbf{c}}$ be as in Remark 3.1. Then, we have that,

$$
\mathbf{G}_{\mathbf{a}, \mathbf{b}, \mathbf{c}} \in \mathcal{C}_{l o c}^{\infty}\left(M_{\mathbf{p}, \mathbf{q}}\right) .
$$

Moreover, if $z^{1}, \ldots, z^{m}$ are local coordinates centered at the singular points, then the following holds.

- If $m \geq 3$, then $\mathbf{G}_{\mathbf{a}, \mathbf{b}, \mathbf{c}}$ blows up like $|z|^{2-2 m}$ at the points points of $p_{1}, \ldots, p_{N}$ and like $|z|^{4-2 m}$ at the points $q_{1}, \ldots, q_{K}$.

- If $m=2$, then $\mathbf{G}_{\mathbf{a}, \mathbf{b}, \mathbf{c}}$ blows up like $|z|^{-2}$ at the points $p_{1}, \ldots, p_{N}$ and like $\log (|z|)$ at the points $q_{1}, \ldots, q_{K}$.

\subsection{Solution of the linearized scalar curvature equation}

In this section, we are going to describe the possible choices for a right inverse of the operator $\mathbb{L}_{\omega}$, in a suitable functional setting. Since this operator is formally selfadjoint and since we are assuming that its kernel is nontrivial, we expect the presence of a nontrivial cokernel. To overcome this difficulty, we are going to consider some appropriate finite dimensional extensions of the natural domain of $\mathbb{L}_{\omega}$, which, according to Theorem 3.1, is given by $C_{\delta}^{4, \alpha}\left(M_{\mathbf{p}, \mathbf{q}}\right)$, with $\delta \in(4-2 m, 0)$ if $m \geq 3$ and $\delta \in(0,1)$ if $m=2$. Building on the analysis of the previous section, we are going to introduce the following deficiency spaces. Given a triple 
of vectors $\boldsymbol{\alpha} \in \mathbb{R}^{K}$ and $\boldsymbol{\beta}, \boldsymbol{\gamma} \in \mathbb{R}^{N}$, we set, for $m \geq 3, l=1, \ldots, K$ and $j=1, \ldots, N$,

$$
\begin{aligned}
W_{\alpha}^{l}= & -\frac{\alpha_{l}}{4(m-2)}\left[\frac{\left|\Gamma_{N+l}\right|}{2(m-1)\left|\mathbb{S}^{2 m-1}\right|} G_{\Delta \Delta}\left(q_{l}, \cdot\right)\right], \\
W_{\boldsymbol{\beta}, \boldsymbol{\gamma}}^{j}= & \beta_{j}\left[\frac{\left|\Gamma_{j}\right|}{2(m-1)\left|\mathbb{S}^{2 m-1}\right|} G_{\Delta}\left(p_{j}, \cdot\right)\right] \\
& -\left(\frac{\gamma_{j}}{4(m-2)}-\frac{s_{\omega}\left(m^{2}-m+2\right) \beta_{j}}{(m-2) m(m+1)}\right) \\
& \times\left[\frac{\left|\Gamma_{j}\right|}{2(m-1)\left|\mathbb{S}^{2 m-1}\right|} G_{\Delta \Delta}\left(p_{j}, \cdot\right)\right],
\end{aligned}
$$

whereas, for $m=2, l=1, \ldots, K$ and $j=1, \ldots, N$, we set,

$$
\begin{aligned}
W_{\alpha}^{l} & =\alpha_{l}\left[\frac{\left|\Gamma_{N+l}\right|}{4\left|\mathbb{S}^{3}\right|} G_{\Delta \Delta}\left(q_{l}, \cdot\right)\right], \\
W_{\boldsymbol{\beta}, \boldsymbol{\gamma}}^{j} & =\beta_{j}\left[\frac{\left|\Gamma_{j}\right|}{\left|\mathbb{S}^{3}\right|} G_{\Delta}\left(p_{j}, \cdot\right)\right]+\left(\frac{\gamma_{j}}{4}-\frac{s_{\omega} \beta_{j}}{6}\right)\left[\frac{\left|\Gamma_{j}\right|}{\left|\mathbb{S}^{3}\right|} G_{\Delta \Delta}\left(p_{j}, \cdot\right)\right] .
\end{aligned}
$$

We are now in the position to define the deficiency spaces,

$$
\mathcal{D}_{\mathbf{q}}(\boldsymbol{\alpha})=\operatorname{span}\left\{W_{\boldsymbol{\alpha}}^{l}: l=1, \ldots, K\right\} \quad \text { and } \quad \mathcal{D}_{\mathbf{p}}(\boldsymbol{\beta}, \boldsymbol{\gamma})=\operatorname{span}\left\{W_{\boldsymbol{\beta}, \boldsymbol{\gamma}}^{j}: j=1, \ldots, N\right\} . .
$$

These are finite dimensional vector spaces and they can be endowed with the following norm. If $V=\sum_{l=1}^{K} V^{l} W_{\boldsymbol{\alpha}}^{l} \in \mathcal{D}_{\mathbf{q}}(\boldsymbol{\alpha})$ and $U=\sum_{j=1}^{N} U^{j} W_{\boldsymbol{\beta}, \boldsymbol{\gamma}}^{j} \in \mathcal{D}_{\mathbf{p}}(\boldsymbol{\beta}, \boldsymbol{\gamma})$, we set,

$$
\|V\|_{\mathcal{D}_{\mathbf{q}}(\boldsymbol{\alpha})}=\sum_{l=1}^{K}\left|V^{l}\right| \text { and }\|U\|_{\mathcal{D}_{\mathbf{p}}(\boldsymbol{\beta}, \boldsymbol{\gamma})}=\sum_{j=1}^{N}\left|U^{j}\right| .
$$

We will also make use of the shorthand notation $\mathcal{D}_{\mathbf{p}, \mathbf{q}}(\boldsymbol{\alpha}, \boldsymbol{\beta}, \boldsymbol{\gamma})$ to indicate the direct sum $\mathcal{D}_{\mathbf{q}}(\boldsymbol{\alpha}) \oplus \mathcal{D}_{\mathbf{p}}(\boldsymbol{\beta}, \boldsymbol{\gamma})$ of the deficiency spaces introduced above, endowed with the obvious norm $\|\cdot\|_{\mathcal{D}_{\mathbf{q}}(\boldsymbol{\alpha})}+\|\cdot\|_{\mathcal{D}_{\mathbf{p}}(\boldsymbol{\beta}, \boldsymbol{\gamma})}$.

To treat the case $m=2$, it is convenient to introduce further finite dimensional extensions of the domain $C_{\delta}^{4, \alpha}\left(M_{\mathbf{p}, \mathbf{q}}\right)$, with $\delta \in(0,1)$. These will be called extra deficiency spaces and they are defined as,

$$
\mathcal{E}_{\mathbf{q}}=\operatorname{span}\left\{\chi_{q_{l}}: l=1, \ldots, K\right\} \text { and } \mathcal{E}_{\mathbf{p}}=\operatorname{span}\left\{\chi_{p_{j}}: j=1, \ldots, N\right\},
$$

where the functions $\chi_{p_{1}}, \ldots, \chi_{p_{N}}, \chi_{q_{1}}, \ldots, \chi_{q_{K}}$ are smooth cutoff functions supported on small balls centered at the points $p_{1}, \ldots, p_{N}, q_{1}, \ldots, q_{K}$ and identically equal to 1 in a neighborhood of these points. Given two functions $X=\sum_{j=1}^{N} X^{j} \chi_{p_{j}} \in \mathcal{E}_{\mathbf{p}}$ and $Y=$ $\sum_{l=1}^{K} Y^{l} \chi_{q_{l}} \in \mathcal{E}_{\mathbf{q}}$, we set,

$$
\|Y\|_{\mathcal{E}_{\mathbf{q}}}=\sum_{l=1}^{K}\left|Y^{l}\right| \text { and }\|X\|_{\mathcal{E}_{\mathbf{p}}}=\sum_{j=1}^{N}\left|X^{j}\right| .
$$

We will also make use of the shorthand notation $\mathcal{E}_{\mathbf{p}, \mathbf{q}}$ to indicate the direct sum $\mathcal{E}_{\mathbf{q}} \oplus \mathcal{E}_{\mathbf{p}}$ of the extra deficiency spaces introduced above, endowed with the obvious norm $\|\cdot\|_{\mathcal{E}_{\mathbf{q}}}+\|\cdot\|_{\mathcal{E}_{\mathbf{p}}}$. Notice that, with these notation, the estimate (3.5) in Theorem 3.1 reads,

$$
\|\tilde{u}\|_{C_{\delta}^{4, \alpha}\left(M_{\mathbf{p}, \mathbf{q}}\right)}+\|\stackrel{\circ}{u}\|_{\mathcal{E}_{\mathbf{p}, \mathbf{q}}} \leq C\|f\|_{C_{\delta-4}^{0, \alpha}}
$$


where $u=\tilde{u}+\stackrel{\circ}{u} \in C_{\delta}^{4, \alpha}\left(M_{\mathbf{p}, \mathbf{q}}\right) \oplus \mathcal{E}_{\mathbf{p}, \mathbf{q}}$ and $f \in C_{\delta-4}^{0, \alpha}\left(M_{\mathbf{p}, \mathbf{q}}\right)$ are functions satisfying the equation $\mathbb{L}_{\omega}[u]=f$ as well as the orthogonality condition (3.3) and $\delta \in(0,1)$.

We recall that we have assumed that the bounded kernel of $\mathbb{L}_{\omega}$ is $(d+1)$-dimensional and that it is spanned by $\left\{\varphi_{0}, \varphi_{1}, \ldots, \varphi_{d}\right\}$, where $\varphi_{0} \equiv 1$ and $\varphi_{1}, \ldots, \varphi_{d}$, with $d \geq 1$, is a collection of mutually $L^{2}(M)$-orthogonal smooth functions with zero mean and $L^{2}(M)$-norm equal to 1 . Given a triple of vectors $\boldsymbol{\alpha} \in \mathbb{R}^{K}$ and $\boldsymbol{\beta}, \boldsymbol{\gamma} \in \mathbb{R}^{N}$, it is convenient to introduce the following matrices,

$$
\begin{aligned}
\Xi_{i l}(\boldsymbol{\alpha}) & :=\alpha_{l} \varphi_{i}\left(q_{l}\right), \text { for } i=1 \ldots, d \text { and } l=1, \ldots, K, \\
\Theta_{i j}(\boldsymbol{\beta}, \boldsymbol{\gamma}) & :=\beta_{j} \Delta \varphi_{i}\left(p_{j}\right)+\gamma_{j} \varphi_{i}\left(p_{j}\right), \text { for } i=1 \ldots, d \text { and } j=1, \ldots, N .
\end{aligned}
$$

These will help us in formulating our nondegeneracy assumption. We are now in the position to state the main results of our linear analysis on the base orbifold.

Theorem 3.4 Let $(M, g, \omega)$ be a compact Kcsc orbifold of complex dimension $m \geq 2$ and let $\operatorname{Ker}\left(\mathbb{L}_{\omega}\right)=\operatorname{span}\left\{\varphi_{0}, \varphi_{1}, \ldots, \varphi_{d}\right\}$. Assume that $(N+K) \geq d$ and that the following nondegeneracy condition is satisfied: a triple of vectors $\boldsymbol{\alpha} \in \mathbb{R}^{K}$ and $\boldsymbol{\beta}, \boldsymbol{\gamma} \in \mathbb{R}^{N}$ is given such that the $d \times(N+K)$ matrix,

$$
\left(\left(\Xi_{i l}(\boldsymbol{\alpha})\right)_{\substack{1 \leq i \leq d \\ 1 \leq l \leq K}} \mid\left(\Theta_{i j}(\boldsymbol{\beta}, \boldsymbol{\gamma})\right)_{\substack{1 \leq i \leq d \\ 1 \leq j \leq N}}\right),
$$

has rank $d$. Then, the following holds.

- If $m \geq 3$, then for every $f \in C_{\delta-4}^{0, \alpha}\left(M_{\mathbf{p}, \mathbf{q}}\right)$ with $\delta \in(4-2 m, 0)$, there exist real number $v$ and a function,

$$
u=\tilde{u}+\widehat{u} \in C_{\delta}^{4, \alpha}\left(M_{\mathbf{p}, \mathbf{q}}\right) \oplus \mathcal{D}_{\mathbf{p}, \mathbf{q}}(\boldsymbol{\alpha}, \boldsymbol{\beta}, \boldsymbol{\gamma})
$$

such that,

$$
\mathbb{L}_{\omega} u+v=f, \text { in } M_{\mathbf{p}, \mathbf{q}} .
$$

Moreover, there exists a positive constant $C=C(\boldsymbol{\alpha}, \boldsymbol{\beta}, \boldsymbol{\gamma}, \delta)>0$ such that,

$$
|v|+\|\tilde{u}\|_{C_{\delta}^{4, \alpha}\left(M_{\mathbf{p}, \mathbf{q}}\right)}+\|\widehat{u}\|_{\mathcal{D}_{\mathbf{p}, \mathbf{q}}(\boldsymbol{\alpha}, \boldsymbol{\beta}, \boldsymbol{\gamma})} \leq C\|f\|_{\mathcal{C}_{\delta-4}^{0, \alpha}\left(M_{\mathbf{p}, \mathbf{q}}\right)} .
$$

- If $m=2$, then for every $f \in C_{\delta-4}^{0, \alpha}\left(M_{\mathbf{p}, \mathbf{q}}\right)$ with $\delta \in(0,1)$, there exist real number $v$ and a function,

$$
u=\tilde{u}+\stackrel{\circ}{u}+\widehat{u} \in C_{\delta}^{4, \alpha}\left(M_{\mathbf{p}, \mathbf{q}}\right) \oplus \mathcal{E}_{\mathbf{p}, \mathbf{q}} \oplus \mathcal{D}_{\mathbf{p}, \mathbf{q}}(\boldsymbol{\alpha}, \boldsymbol{\beta}, \boldsymbol{\gamma}),
$$

such that,

$$
\mathbb{L}_{\omega} u+v=f, \text { in } M_{\mathbf{p}, \mathbf{q}} .
$$

Moreover, there exists a positive constant $C=C(\boldsymbol{\alpha}, \boldsymbol{\beta}, \boldsymbol{\gamma}, \delta)>0$ such that,

$$
|v|+\|\widetilde{u}\|_{C_{\delta}^{4, \alpha}\left(M_{\mathbf{p}, \mathbf{q}}\right)}+\|\stackrel{\circ}{u}\|_{\mathcal{E}_{\mathbf{p}, \mathbf{q}}}+\|\widehat{u}\|_{\mathcal{D}_{\mathbf{p}, \mathbf{q}}(\boldsymbol{\alpha}, \boldsymbol{\beta}, \boldsymbol{\gamma})} \leq C\|f\|_{C_{\delta-4}^{0, \alpha}\left(M_{\mathbf{p}, \mathbf{q}}\right)} .
$$

Proof We only prove the statement in the case $m \geq 3$, since it is completely analogous in the other case. For sake of simplicity we assume $\boldsymbol{\alpha}=\mathbf{0} \in \mathbb{R}^{K}$, so that the nondegeneracy condition becomes equivalent to the requirement that the matrix,

$$
\left(\Theta_{i j}(\boldsymbol{\beta}, \boldsymbol{\gamma})\right) \underset{\substack{1 \leq i \leq d \\ 1, \leq j \leq N}}{\substack{1, \leq \\ \text {. }}}
$$


has full rank. Under these assumptions, the deficiency space $\mathcal{D}_{\mathbf{p}, \mathbf{q}}(\boldsymbol{\alpha}, \boldsymbol{\beta}, \boldsymbol{\gamma})$ reduces to $\mathcal{D}_{\mathbf{p}}(\boldsymbol{\beta}, \boldsymbol{\gamma})$. In order to split our problem, it is convenient to set,

$$
f^{\perp}=f-\frac{1}{\operatorname{Vol}_{\omega}(M)} \int_{M} f d \mu_{\omega}-\sum_{i=1}^{d} \varphi_{i} \int_{M} f \varphi_{i} d \mu_{\omega}
$$

so that $f^{\perp}$ satisfies the orthogonality conditions (3.3). By Theorem 3.1, we obtain the existence of a function $u^{\perp} \in C_{\delta}^{4, \alpha}\left(M_{\mathbf{p}, \mathbf{q}}\right)$, which satisfies the equation,

$$
\mathbb{L}_{\omega}\left[u^{\perp}\right]=f^{\perp},
$$

together with the orthogonality conditions (3.3) and the desired estimate (3.4). To complete the resolution of Eq. (3.12), we set,

$$
f_{0}=\frac{1}{\operatorname{Vol}_{\omega}(M)} \int_{M} f d \mu_{\omega} \text { and } f_{i}=\int_{M} f \varphi_{i} d \mu_{\omega}, \quad \text { for } i=1, \ldots, d
$$

Recalling the definition of $\Theta_{i j}(\boldsymbol{\beta}, \boldsymbol{\gamma})$ and using the nondegeneracy condition, we select a solution $\left(v, U_{1}, \ldots, U_{N}\right) \in \mathbb{R}^{N+1}$ to the following system of linear balancing conditions,

$$
\begin{gathered}
f_{i}+\sum_{j=1}^{N} U^{j}\left[\beta_{j}\left(\Delta \varphi_{i}\right)\left(p_{j}\right)+\gamma_{j} \varphi_{i}\left(p_{j}\right)\right]=0, \quad i=1, \ldots, d, \\
f_{0} \operatorname{Vol}_{\omega}(M)+\sum_{j=1}^{N} U^{j} \gamma_{j}=v \operatorname{Vol}_{\omega}(M) .
\end{gathered}
$$

It is worth pointing out that in general this choice is not unique, since it depends in the choice of a right inverse for the matrix $\Theta_{i j}(\boldsymbol{\beta}, \boldsymbol{\gamma})$. Theorem 3.2 implies then the existence of a distribution $U \in \mathscr{D}^{\prime}(M)$ which satisfies,

$$
\mathbb{L}_{\omega}[U]+v=\sum_{i=0}^{d} f_{i} \varphi_{i}+\sum_{j=1}^{N} U^{j} \beta_{j} \Delta \delta_{p_{j}}+\sum_{j=1}^{N} U^{j} \gamma_{j} \delta_{p_{j}}, \quad \text { in } M .
$$

Arguing as in Proposition 3.3, it is not hard to show that $U \in C_{l o c}^{\infty}\left(M_{\mathbf{p}}\right)$. In particular the function $u^{\perp}+U \in C_{l o c}^{4, \alpha}\left(M_{\mathbf{p}}\right)$ satisfies the equation,

$$
\mathbb{L}_{\omega}\left[u^{\perp}+U\right]+v=f, \text { in } M_{\mathbf{p}} .
$$

To complete the proof of our statement, we need to describe the local structure of $U$ in more details. First, we observe that, by the very definition of the deficiency spaces, one has,

$$
\mathbb{L}_{\omega}\left[W_{\boldsymbol{\beta}, \boldsymbol{\gamma}}^{j}\right]=\beta_{j} \Delta \delta_{p_{j}}+\gamma_{j} \delta_{p_{j}}+V_{\boldsymbol{\beta}, \boldsymbol{\gamma}}^{j},
$$


where, for every $j=1, \ldots, N$, the function $V_{\boldsymbol{\beta}, \boldsymbol{\gamma}}^{j}$ is in $C^{\infty}(M)$. Combining this fact with the linear balancing conditions, we deduce that,

$$
\begin{aligned}
\mathbb{L}_{\omega}\left[U-\sum_{j=1}^{N} U^{j} W_{\boldsymbol{\beta}, \boldsymbol{\gamma}}^{j}\right]= & f_{0}-v+\sum_{i=1}^{d} f_{i} \phi_{i}-\sum_{j=1}^{N} U^{j} V_{\boldsymbol{\beta}, \boldsymbol{\gamma}}^{j} \\
= & \frac{1}{\operatorname{Vol}_{\omega}(M)} \sum_{j=1}^{N} U^{j} \gamma_{j}-\sum_{i=1}^{d} \sum_{j=1}^{N} U^{j} \Theta_{i j}(\boldsymbol{\beta}, \boldsymbol{\gamma}) \phi_{i} \\
& -\sum_{j=1}^{N} U^{j} V_{\boldsymbol{\beta}, \boldsymbol{\gamma}}^{j}
\end{aligned}
$$

By the definition of $V_{\boldsymbol{\beta}, \boldsymbol{\gamma}}^{j}$ it follows that,

$$
\int_{M} V_{\boldsymbol{\beta}, \boldsymbol{\gamma}}^{j} \phi_{0} d \mu_{\omega}=-\gamma_{j} \text { and } \int_{M} V_{\boldsymbol{\beta}, \boldsymbol{\gamma}}^{j} \phi_{i} d \mu_{\omega}=-\Theta_{i j}(\boldsymbol{\beta}, \boldsymbol{\gamma}),
$$

and thus, it is easy to check the right hand side of the equation above is orthogonal to $\operatorname{ker}\left(\mathbb{L}_{\omega}\right)$. Hence, using Theorem 3.1 and by the elliptic regularity, we deduce the existence of a smooth function $\bar{u} \in C^{\infty}(M)$ which satisfies,

$$
\mathbb{L}_{\omega}[\bar{u}]=\frac{1}{\operatorname{Vol}_{\omega}(M)} \sum_{j=1}^{N} U^{j} \gamma_{j}-\sum_{i=1}^{d} \sum_{j=1}^{N} U^{j} \Theta_{i j}(\boldsymbol{\beta}, \boldsymbol{\gamma}) \phi_{i}-\sum_{j=1}^{N} U^{j} V_{\boldsymbol{\beta}, \boldsymbol{\gamma}}^{j}, \quad \text { in } M .
$$

Setting $\widehat{u}=\sum_{j=1}^{N} U^{j} W_{\boldsymbol{\beta}, \boldsymbol{\gamma}}^{j}$, we have obtained that $\mathbb{L}_{\omega}[U]=\mathbb{L}_{\omega}[\widehat{u}+\bar{u}]$, hence,

$$
\mathbb{L}_{\omega}\left[u^{\perp}+\bar{u}+\widehat{u}\right]+v=f, \quad \text { in } M_{\mathbf{p}},
$$

with $\tilde{u}=\left(u^{\perp}+\bar{u}\right) \in C_{\delta}^{4, \alpha}\left(M_{\mathbf{p}}\right)$ and $\widehat{u} \in \mathcal{D}_{\mathbf{p}}(\boldsymbol{\beta}, \boldsymbol{\gamma})$. Moreover, combining the estimate (3.4) with our construction, it is clear that, for suitable positive constants $C_{0}, \ldots, C_{3}$, possibly depending on $\boldsymbol{\beta}, \boldsymbol{\gamma}$ and $\delta$, it holds,

$$
\begin{aligned}
\|u\|_{C_{\delta}^{4, \alpha}\left(M_{\mathbf{p}}\right) \oplus \mathcal{D}_{\mathbf{p}}(\boldsymbol{\beta}, \boldsymbol{\gamma})} & =\|\tilde{u}\|_{C_{\delta}^{4, \alpha}\left(M_{\mathbf{p}}\right)}+\|\widehat{u}\|_{\mathcal{D}_{\mathbf{p}}(\boldsymbol{\beta}, \boldsymbol{\gamma})} \\
& \leq\left\|u^{\perp}\right\|_{C_{\delta}^{4, \alpha}\left(M_{\mathbf{p}}\right)}+\|\bar{u}\|_{C_{\delta}^{4, \alpha}\left(M_{\mathbf{p}}\right)}+\|\widehat{u}\|_{\mathcal{D}_{\mathbf{p}}(\boldsymbol{\beta}, \boldsymbol{\gamma})} \\
& \leq C_{0}\left\|f^{\perp}\right\|_{C_{\delta-4}^{0, \alpha}\left(M_{\mathbf{p}}\right)}+C_{1} \sum_{j=1}^{N}\left|U^{j}\right| \\
& \leq C_{2}\left(\left\|f^{\perp}\right\|_{C_{\delta-4}^{0, \alpha}\left(M_{\mathbf{p}}\right)}+\sum_{i=1}^{d}\left|f_{i}\right|\right) \\
& \leq C_{3}\|f\|_{C_{\delta-4}^{0, \alpha}\left(M_{\mathbf{p}}\right)},
\end{aligned}
$$

which is the desired estimate. Finally, we observe that the constant $v$ as well can be easily estimated in terms of the norm of $f$. This concludes the proof of the theorem.

Remark 3.2 In other words, with the notations introduced in the proof of the previous theorem, we have proven that, for $m \geq 3$ and $\delta \in(4-2 m, 0)$, the operator,

$$
\begin{aligned}
\mathbb{L}_{\boldsymbol{\alpha}, \boldsymbol{\beta}, \boldsymbol{\gamma}}^{(\delta)}: C_{\delta}^{4, \alpha}\left(M_{\mathbf{p}, \mathbf{q}}\right) \oplus \mathcal{D}_{\mathbf{p}, \mathbf{q}}(\boldsymbol{\alpha}, \boldsymbol{\beta}, \boldsymbol{\gamma}) \times \mathbb{R} & \longrightarrow C_{\delta-4}^{0, \alpha}\left(M_{\mathbf{p}, \mathbf{q}}\right) \\
(\tilde{u}+\widehat{u}, v) & \longmapsto \mathbb{L}_{\omega}[\tilde{u}+\widehat{u}]+v,
\end{aligned}
$$


with $\boldsymbol{\beta}, \boldsymbol{\gamma}$ and $\boldsymbol{\alpha}$ satisfying the nondegeneracy condition, admits a (in general not unique) bounded right inverse,

$$
\mathbb{J}_{\boldsymbol{\alpha}, \boldsymbol{\beta}, \boldsymbol{\gamma}}^{(\delta)}: C_{\delta-4}^{0, \alpha}\left(M_{\mathbf{p}, \mathbf{q}}\right) \longrightarrow C_{\delta}^{4, \alpha}\left(M_{\mathbf{p}, \mathbf{q}}\right) \oplus \mathcal{D}_{\mathbf{p}, \mathbf{q}}(\boldsymbol{\alpha}, \boldsymbol{\beta}, \boldsymbol{\gamma}) \times \mathbb{R},
$$

so that $\left(\mathbb{L}_{\boldsymbol{\alpha}, \boldsymbol{\beta}, \boldsymbol{\gamma}}^{(\delta)} \circ \mathbb{J}_{\boldsymbol{\alpha}, \boldsymbol{\beta}, \boldsymbol{\gamma}}^{(\delta)}\right)[f]=f$, for every $f \in C_{\delta-4}^{0, \alpha}\left(M_{\mathbf{p}, \mathbf{q}}\right)$ and,

$$
\left\|\mathbb{J}_{\boldsymbol{\alpha}, \boldsymbol{\beta}, \boldsymbol{\gamma}}^{(\delta)}[f]\right\|_{C_{\delta}^{4, \alpha}\left(M_{\mathbf{p}, \mathbf{q}}\right) \oplus \mathcal{D}_{\mathbf{p}, \mathbf{q}}(\boldsymbol{\alpha}, \boldsymbol{\beta}, \boldsymbol{\gamma}) \times \mathbb{R}} \leq C\|f\|_{C_{\delta-4}^{0, \alpha}\left(M_{\mathbf{p}, \mathbf{q}}\right)} .
$$

Of course, the analogous conclusion holds in the case $m=2$.

\section{Linear analysis on ALE manifolds}

We now reproduce an analysis similar to the one just completed on the base orbifold on our model ALE resolutions of isolated singularities. We define also in this setting weighted Hölder spaces. Since we will use duality arguments we introduce also weighted Sobolev spaces. Let $\left(X_{\Gamma}, h, \eta\right)$ be an $A L E$ Kähler resolution of isolated singularity and set,

$$
X_{\Gamma, R_{0}}=\pi^{-1}\left(B_{R_{0}}\right) .
$$

where $\pi: X_{\Gamma} \rightarrow \mathbb{C}^{m} / \Gamma$ is the canonical projection. This can be thought as the counterpart in $X_{\Gamma}$ of $M_{r_{0}}$ in $M$. For $\delta \in \mathbb{R}$ and $\alpha \in(0,1)$, the weighted Hölder space $C_{\delta}^{k, \alpha}\left(X_{\Gamma}\right)$ is the set of functions $f \in C_{l o c}^{k, \alpha}\left(X_{\Gamma}\right)$ such that,

$$
\|f\|_{C_{\delta}^{k, \alpha}\left(X_{\Gamma}\right)}:=\|f\|_{C^{k, \alpha}\left(X_{\Gamma, R_{0}}\right)}+\sup _{R \geq R_{0}} R^{-\delta}\|f(R \cdot)\|_{C^{k, \alpha}\left(B_{1} \backslash B_{1 / 2}\right)}<+\infty .
$$

In order to define weighted Sobolev spaces we have to introduce a distance-like function $\gamma \in C_{l o c}^{\infty}\left(X_{\Gamma}\right)$ defined as,

$$
\gamma(p):=\chi(p)+(1-\chi(p))|x(p)| \quad p \in X_{\Gamma},
$$

with $\chi$ a smooth cutoff function identically 1 on $X_{\Gamma, R_{0}}$ and identically 0 on $X_{\Gamma} \backslash X_{\Gamma, 2 R_{0}}$. For $\delta \in \mathbb{R}$, the weighted Sobolev space $W_{\delta}^{k, 2}\left(X_{\Gamma}\right)$ is the set of functions $f \in L_{l o c}^{1}\left(X_{\Gamma}\right)$ such that,

$$
\|f\|_{W_{\delta}^{k, 2}\left(X_{\Gamma}\right)}:=\sqrt{\sum_{j=0}^{k} \int_{X}\left|\gamma^{-\delta-m+j} \nabla^{(j)} f\right|_{\eta}^{2} d \mu_{\eta}}<+\infty,
$$

where,

$$
\nabla^{(j)} f:=\underbrace{\nabla \circ \cdots \circ \nabla}_{j \text { times }} f .
$$

We recall now the natural duality between weighted spaces,

$$
\langle\cdot \mid \cdot\rangle_{\eta}: L_{\delta}^{2}\left(X_{\Gamma}\right) \times L_{-2 m-\delta}^{2}\left(X_{\Gamma}\right) \rightarrow \mathbb{R},
$$

defined as,

$$
\langle f \mid g\rangle_{\eta}:=\int_{X} f g d \mu_{\eta}
$$


Remark 4.1 We note that a function $f \in W_{\delta}^{k, 2}\left(X_{\Gamma}\right) \cap C_{l o c}^{\infty}\left(X_{\Gamma}\right)$ on the set $X_{\Gamma} \backslash X_{\Gamma, R_{0}}$ beheaves like,

$$
\left.f\right|_{X_{\Gamma} \backslash X_{\Gamma, R_{0}}}(p)=\mathcal{O}\left(|x(p)|^{\delta^{\prime}}\right) \quad \text { for dome } \delta^{\prime}<\delta .
$$

and a function $f \in C^{k \alpha}\left(X_{\Gamma}\right)$ on the set $X \backslash X_{\Gamma, R_{0}}$ typically beheaves like,

$$
\left.f\right|_{X_{\Gamma} \backslash X_{\Gamma, R_{0}}}(p)=\mathcal{O}\left(|x(p)|^{\delta}\right) .
$$

We also note that for every $\delta^{\prime}<\delta$ we have the inclusion,

$$
C_{\delta}^{k, \alpha}\left(X_{\Gamma}\right) \subseteq W_{\delta^{\prime}}^{k, 2}\left(X_{\Gamma}\right) .
$$

The main task of this section is to solve the linearized constant scalar curvature equation,

$$
\mathbb{L}_{\eta} u=f \text {. }
$$

We recall that by (2.2),

$$
\mathbb{L}_{\eta} u=\Delta_{\eta}^{2} u+4\left\langle\rho_{\eta} \mid i \partial \bar{\partial} u\right\rangle .
$$

and, since $\left(X_{\Gamma}, h, \eta\right)$ is scalar flat, $\mathbb{L}_{\eta}$ is formally self-adjoint. We also notice that if $\left(X_{\Gamma}, h, \eta\right)$ is Ricci-flat, the operator $\mathbb{L}_{\eta}$ reduces to the $\eta$ bi-Laplacian operator. Since we want to study the operator $\mathbb{L}_{\eta}$ on weighted spaces we have to be careful on the choice of weights. Indeed to have Fredholm properties we must avoid the indicial roots at infinity of $\mathbb{L}_{\eta}$ that, thanks to the decay of the metric, coincide with those of euclidean bi-Laplace operator $\Delta^{2}$. We recall that the set of indicial roots at infinity for $\Delta^{2}$ on $\mathbb{C}^{m}$ is $\mathbb{Z} \backslash\{5-2 m, \ldots,-1\}$ for $m \geq 3$ and $\mathbb{Z}$ for $m=2$. Let $\delta \in \mathbb{R}$ with,

$$
\delta \notin \mathbb{Z} \backslash\{5-2 m, \ldots,-1\} .
$$

for $m \geq 3$ and $\delta \notin \mathbb{Z}$ for $m=2$, then the operator,

$$
\mathbb{L}_{\eta}^{(\delta)}: W_{\delta}^{4,2}\left(X_{\Gamma}\right) \rightarrow L_{\delta-4}^{2}\left(X_{\Gamma}\right)
$$

is Fredholm and its cokernel is the kernel of its adjoint under duality (4.1),

$$
\mathbb{L}_{\eta}^{(-2 m-\delta)}: W_{-2 m-\delta}^{4,2}\left(X_{\Gamma}\right) \rightarrow L_{-2 m-4-\delta}^{2}\left(X_{\Gamma}\right) .
$$

For $A L E$ Kähler manifolds a result analogous to Proposition 3.1 holds true.

Proposition 4.1 Let $\left(X_{\Gamma}, h, \eta\right)$ a scalar flat ALE Kähler resolution. If $m \geq 3$ and $\delta \in$ $(4-2 m, 0)$, then,

$$
\mathbb{L}_{\eta}^{(\delta)}: C_{\delta}^{4, \alpha}\left(X_{\Gamma}\right) \longrightarrow C_{\delta-4}^{0, \alpha}\left(X_{\Gamma}\right),
$$

is invertible. If $m=2$ and $\delta \in(0,1)$, then,

$$
\mathbb{L}_{\eta}^{(\delta)}: C_{\delta}^{4, \alpha}\left(X_{\Gamma}\right) \longrightarrow C_{\delta-4}^{0, \alpha}\left(X_{\Gamma}\right)
$$

is surjective with one dimensional kernel spanned by the constant function.

Remark 4.2 Rephrasing Proposition 4.1 we can say that for $\delta \in(4-2 m)$ if $m \geq 3$ and $\delta \in(0,1)$ if $m=2$ the operator,

$$
\mathbb{L}_{\eta}^{(\delta)}: C_{\delta}^{4, \alpha}\left(X_{\Gamma}\right) \longrightarrow C_{\delta-4}^{0, \alpha}\left(X_{\Gamma}\right) .
$$

has a continuous right inverse,

$$
\mathbb{J}^{(\delta)}: C_{\delta-4}^{0, \alpha}\left(X_{\Gamma}\right) \longrightarrow C_{\delta}^{4, \alpha}\left(X_{\Gamma}\right) .
$$


The proof of the above result follows standard lines (see e.g. Theorem 10.2.1 and Proposition 11.1.1 in [23]). We focus now on the asymptotic expansions of various operators on $A L E$ manifolds.

Lemma 4.1 Let $\left(X_{\Gamma}, h, \eta\right)$ be a scalar flat $A L E$-Kähler resolution with e $(\Gamma)=0$. Then on the coordinate chart at infinity we have the following expansions,

- for the inverse of the metric $\eta^{i j}$

$$
\eta^{i \bar{J}}=2\left[\delta^{i \bar{\jmath}}-\frac{2 c_{X_{\Gamma}}(m-1)}{|x|^{2 m}}\left(\delta_{i \bar{j}}-m \frac{\overline{x^{i}} x^{j}}{|x|^{2}}\right)+\mathcal{O}\left(|x|^{-2-2 m}\right)\right] ;
$$

- for the unit normal vector to the sphere $|x|=\rho$

$$
v=\frac{1}{|x|}\left(x^{i} \frac{\partial}{\partial x^{i}}+\overline{x^{i}} \frac{\partial}{\partial \overline{x^{i}}}\right)\left[1+\frac{c_{X_{\Gamma}}(m-1)^{2}}{|x|^{2 m}}+\mathcal{O}\left(|x|^{-2-2 m}\right)\right] ;
$$

- for the laplacian $\Delta_{\eta}$

$$
\Delta_{\eta}=\left[1-\frac{2 c_{X_{\Gamma}}(m-1)}{|x|^{2 m}}\right] \Delta+\left[\frac{8 c_{X_{\Gamma}}(m-1) m}{|x|^{2 m+2}} x^{i} x^{j}+\mathcal{O}\left(|x|^{-2-2 m}\right)\right] \partial_{j} \partial_{\bar{\imath}} .
$$

The proof of the above lemma consists of straightforward computations and is therefore omitted.

We conclude this section with an observation regarding fine mapping properties of,

$$
\mathbb{L}_{\eta}^{(\delta)}: W_{\delta}^{4,2}\left(X_{\Gamma}\right) \rightarrow L_{\delta-4}^{2}\left(X_{\Gamma}\right),
$$

that will be useful in Sect. 5.3 in a crucial point where we show how the nonlinear analysis constrains the choice of balancing parameters. In the following proposition we want to solve the equation,

$$
\mathbb{L}_{\eta}[u]=f,
$$

with $f \in L_{\delta-4}^{2}\left(X_{\Gamma}\right)\left(C_{\delta-4}^{0, \alpha}\left(X_{\Gamma}\right)\right)$. In general, when $\delta \in(2-2 m, 4-2 m)$, the indicial root $3-2 m$ imposes to the solution $u$ to have a component with asymptotic growth $|x|^{3-2 m}$. The keypoint of Proposition is that if $\Gamma$ is non trivial this doesn't occur.

Proposition 4.2 Let $\left(X_{\Gamma}, h, \eta\right)$ be a scalar-flat ALE Kähler resolution with $e_{X_{\Gamma}}=0$ and nontrivial $\Gamma \triangleleft U(m)$. For $\delta \in(2-2 m, 4-2 m)$, the equation,

$$
\mathbb{L}_{\eta}[u]=f
$$

with $f \in L_{\delta-4}^{2}\left(X_{\Gamma}\right)$ (respectively $f \in C_{\delta-4}^{0, \alpha}\left(X_{\Gamma}\right)$ ) is solvable for $u \in W_{\delta}^{4,2}\left(X_{\Gamma}\right)$ (respectively $u \in C_{\delta}^{4, \alpha}\left(X_{\Gamma}\right)$ ) if and only if,

$$
\int_{X_{\Gamma}} f d \mu_{\eta}=0
$$

Proof We are going to prove the following characterization:

$$
\mathbb{L}_{\eta}^{(\delta)}\left[W_{\delta}^{4,2}\left(X_{\Gamma}\right)\right]=\left\{f \in L_{\delta}^{2}\left(X_{\Gamma}\right) \mid \int_{X_{\Gamma}} f d \mu_{\eta}=0\right\} .
$$

Since $\mathbb{L}_{\eta}$ is formally selfadjoint we can identify, via duality (4.1), the cokernel of,

$$
\mathbb{L}_{\eta}^{(\delta)}: W_{\delta}^{4,2}\left(X_{\Gamma}\right) \rightarrow L_{\delta-4}^{2}\left(X_{\Gamma}\right) \quad \delta \in(2-2 m, 4-2 m),
$$


with the kernel of,

$$
\mathbb{L}_{\eta}^{(-2 m-\delta)}: W_{-2 m-\delta}^{4,2}\left(X_{\Gamma}\right) \rightarrow L_{-2 m-4-\delta}^{2}\left(X_{\Gamma}\right) .
$$

We want to identify generators of this kernel. Let then $u \in W_{\delta}^{4,2}\left(X_{\Gamma}\right)$ such that,

$$
\mathbb{L}_{\eta}[u]=0,
$$

with $\delta \in(0,2)$,we want ot prove that $u \equiv c_{0}$ for some $c_{0} \in \mathbb{R}$. By standard elliptic regularity we have that $u \in C_{\text {loc }}^{\omega}\left(X_{\Gamma}\right)$. On $X_{\Gamma} \backslash X_{\Gamma, R}$ we consider the Fourier expansion of $u$,

$$
u=\sum_{k=0}^{+\infty} u^{(k)}(|x|) \phi_{k}
$$

with $u^{(k)} \in C_{\delta}^{n, \alpha}([R,+\infty))$ for any $n \in \mathbb{N}$ and this sum is $C^{n, \alpha}$-convergent on compact sets. Then, using expansions (4.2), (4.3), (4.4), we have on $X_{\Gamma} \backslash X_{\Gamma, R}$,

$0=\Delta_{\eta}^{2}[u]=\sum_{k=0}^{+\infty} \Delta^{2}\left[u^{(k)}(|x|) \phi_{k}\right]+|x|^{-2 m} L_{4}[u]+|x|^{-1-2 m} L_{3}[u]+|x|^{-2-2 m} L_{2}[u]$.

where the $L_{k}$ 's are differential operators of order $k$ and uniformly bounded coefficients. The equation,

$$
\sum_{k=0}^{+\infty} \Delta^{2}\left[u^{(k)}(|x|) \phi_{k}\right]=-|x|^{-2 m} L_{4}[u]-|x|^{-1-2 m} L_{3}[u]-|x|^{-2-2 m} L_{2}[u],
$$

implies

$$
\Delta^{2}\left[u^{(k)} \phi_{k}\right] \in C_{\delta-2 m-4}^{n, \alpha}\left(X_{\Gamma} \backslash X_{\Gamma, R}\right) \text { for } k \geq 0 .
$$

Suppose by contradiction that,

$$
\limsup _{|x| \rightarrow+\infty}|u|>0
$$

Since $u^{(k)} \phi_{k} \in C_{\delta}^{n, \alpha}\left(X_{\Gamma} \backslash X_{\Gamma, R}\right)$ the only possibilities are,

$$
\begin{aligned}
& u^{(0)}(|x|)=c_{0}+v_{0}(|x|) \\
& u^{(1)}(|x|)=\left(|x|+v_{1}(|x|)\right) \phi_{1},
\end{aligned}
$$

with $v_{0}, v_{1} \in C_{\delta-2 m}^{n, \alpha}([R,+\infty))$ and $c_{0} \in \mathbb{R}$. But there are not $\phi_{1}$ that are $\Gamma$-invariant (see Remark 2.3) since $\Gamma$ is nontrivial, so the only possibility is that,

$$
u^{(0)}(|x|)=c_{0}+v_{0}(|x|) .
$$

We now show that $u$ is actually constant, indeed $u-c_{0} \in C_{\delta-2 m}^{n, \alpha}(X)$ and,

$$
\mathbb{L}_{\eta}\left[u-c_{0}\right]=\Delta_{\eta}^{2}\left[u-c_{0}\right]=0,
$$

so by Proposition 4.1 we can conclude,

$$
u-c_{0} \equiv 0 .
$$

The proposition now follows immediately. 


\section{Nonlinear analysis}

In this section we collect all the estimates needed in the proof of Theorem1.1. As in [4] and [5] we produce Kcsc metrics on orbifolds with boundary which we believe could be of independent interest (Propositions 5.1, 5.2).

From now on we will assume that the points in $\mathbf{p} \subset M$ have resolutions which are Ricci-Flat ALE Kähler manifold.

Given $\varepsilon$ sufficiently small we look at the truncated orbifolds $M_{r_{\varepsilon}}$ and $X_{\Gamma_{j}, R_{\varepsilon}}$ for $j=1, \ldots, N$ where we impose the following relations:

$$
r_{\varepsilon}=\varepsilon^{\frac{2 m-1}{2 m+1}}=\varepsilon R_{\varepsilon}
$$

We want to construct families of Kcsc metrics on $M_{r_{\varepsilon}}$ and $X_{\Gamma_{j}, R_{\varepsilon}}$ perturbing Kähler potentials of $\omega$ and $\eta_{j}$ 's. We build these perturbations in such a way that they depend on parameters that we call pseudo- boundary data and we can also prescribe, with some freedom, principal asymptotics of the resulting Kcsc metrics. By principal asymptotics we mean the terms of the potentials of the families of Kcsc metrics on $M_{r_{\varepsilon}}$ that near points $p_{j}$ beheave like $|z|^{2-2 m}$ or $|z|^{4-2 m}$ and the terms of the potentials of the families of Kcsc metrics on $X_{\Gamma_{j}, R_{\varepsilon}}$ approaching infinity beheave like $|x|^{2-2 m}$ or $|x|^{4-2 m}$. In a second moment we choose the exact shape of these asymptotics by specifying some free parameters (tuning). The pseudo-boundary data form a particular set of functions on the unit sphere and they are the parameters that rule the behavior of the families of Kcsc metrics at the boundaries $\partial M_{r_{\varepsilon}}$ and $\partial X_{\Gamma_{j}, R_{\varepsilon}}$. They are the main tool for gluing the various families of metrics to a unique Kcsc metric on the resulting manifold, indeed their arbitrariness will allow us to perform the procedure of data matching. We call them pseudo-boundary data because they represent small perturbations of the (suitably rescaled) potentials of the Kcsc metrics at the boundaries.

Notation For the rest of the section $\chi_{j}$ will denote a smooth cutoff functions identically equal to 1 on $B_{2 r_{0}}\left(p_{j}\right)$ and identically equal to 0 outside $B_{3 r_{0}}\left(p_{j}\right)$.

\subsection{Pseudo-boundary data and euclidean Biharmonic extensions}

A key technical tool to implement such a strategy is given by using outer (which will be transplanted on the base orbifold) and inner (transplanted on the model) euclidean biharmonic extensions of functions on the unit sphere. We define now the outer biharmonic extensions of functions on the unit sphere. Let $(h, k) \in C^{4, \alpha}\left(\mathbb{S}^{2 m-1}\right) \times C^{4, \alpha}\left(\mathbb{S}^{2 m-1}\right)$ the outer biharmonic extension of $(h, k)$ is the function $H_{h, k}^{o} \in C^{4, \alpha}\left(\mathbb{C}^{m} \backslash B_{1}\right)$ solution fo the boundary value problem,

$$
\begin{cases}\Delta^{2} H_{h, k}^{\text {out }}=0 & \text { on } \mathbb{C}^{m} \backslash B_{1} \\ H_{h, k}^{\text {out }}=h & \text { on } \partial B_{1} \\ \Delta H_{h, k}^{\text {out }}=k & \text { on } \partial B_{1} .\end{cases}
$$

Moreover $H_{h, k}^{\text {out }}$ has the following expansion in Fourier series for $m \geq 3$,

$$
H_{h, k}^{\text {out }}:=\sum_{\gamma=0}^{+\infty}\left(\left(h^{(\gamma)}+\frac{k^{(\gamma)}}{4(m+\gamma-2)}\right)|w|^{2-2 m-\gamma}-\frac{k^{(\gamma)}}{4(m+\gamma-2)}|w|^{4-2 m-\gamma}\right) \phi_{\gamma},
$$


and for $m=2$

$$
\begin{aligned}
H_{h, k}^{\text {out }}:= & h^{(0)}|w|^{-2}+\frac{k^{(0)}}{2} \log (|w|) \\
& +\sum_{\gamma=1}^{+\infty}\left(\left(h^{(\gamma)}+\frac{k^{(\gamma)}}{4 \gamma}\right)|w|^{-2-\gamma}-\frac{k^{(\gamma)}}{4 \gamma}|w|^{-\gamma}\right) \phi_{\gamma} .
\end{aligned}
$$

Remark 5.1 In the sequel we will take $\Gamma$-invariant $(h, k) \in C^{4, \alpha}\left(\mathbb{S}^{2 m-1}\right) \times C^{4, \alpha}\left(\mathbb{S}^{2 m-1}\right)$ and by the Remark 2.3 we will have no terms with $\phi_{1}$ in the formulas (5.1) and (5.2) for nontrivial $\Gamma$.

We define also the inner biharmonic extensions of functions on the unit sphere. Let $(\tilde{h}, \tilde{k}) \in C^{4, \alpha}\left(\mathbb{S}^{2 m-1}\right) \times C^{2, \alpha}\left(\mathbb{S}^{2 m-1}\right)$, the biharmonic extension $H_{\tilde{h}, \tilde{k}}^{i n}$ on $B_{1}$ of $(\tilde{h}, \tilde{k})$ is the function $H_{\tilde{h}, \tilde{k}}^{i n} \in C^{4, \alpha}\left(\overline{B_{1}}\right)$ given by the solution of the boundary value problem,

$$
\begin{cases}\Delta^{2} H_{\tilde{h}, \tilde{k}}^{i n}=0 & w \in B_{1} \\ H_{\tilde{h}, \tilde{k}}^{i n}=\tilde{h} & w \in \partial B_{1} . \\ \Delta H_{\tilde{h}, \tilde{k}}^{i n}=\tilde{k} & w \in \partial B_{1}\end{cases}
$$

The function $H_{\tilde{h}, \tilde{k}}^{i n}$ has moreover the expansion,

$$
H_{\tilde{h}, \tilde{k}}^{i n}(w)=\sum_{\gamma=0}^{+\infty}\left(\left(\tilde{h}^{(\gamma)}-\frac{\tilde{k}^{(\gamma)}}{4(m+\gamma)}\right)|w|^{\gamma}+\frac{\tilde{k}^{(\gamma)}}{4(m+\gamma)}|w|^{\gamma+2}\right) \phi_{\gamma}
$$

Remark 5.2 Again, if the group $\Gamma$ is non trivial and for $\Gamma$-invariant $(h, k)$, by Remark 2.3 , there will be no $\phi_{1}$-term in the above summations. So we will have,

$$
H_{h, k}^{i n}=\left(\tilde{h}^{(0)}-\frac{\tilde{k}^{(0)}}{4 m}\right)+\frac{\tilde{k}^{(0)}}{4 m}|w|^{2}+\sum_{\gamma=2}^{+\infty}\left(\left(\tilde{h}^{(\gamma)}-\frac{\tilde{k}^{(\gamma)}}{4(m+\gamma)}\right)|w|^{\gamma}+\frac{\tilde{k}^{(\gamma)}}{4(m+\gamma)}|w|^{\gamma+2}\right) \phi_{\gamma} .
$$

As in $[4,5]$ we introduce some functional spaces that will be needed in the sequel that will naturally work as "space of parameters" for our construction:

$$
\begin{aligned}
\mathcal{B}_{j} & :=C^{4, \alpha}\left(\mathbb{S}^{2 m-1} / \Gamma_{j}\right) \times C^{2, \alpha}\left(\mathbb{S}^{2 m-1} / \Gamma_{j}\right) \\
\mathcal{B} & :=\prod_{j=1}^{N} \mathcal{B}_{j} \\
\mathcal{B}(\kappa, \delta) & :=\left\{(\mathbf{h}, \mathbf{k}) \in \mathcal{B} \mid\left\|h_{j}^{(0)}, k_{j}^{(0)}\right\|_{\mathcal{B}_{j}} \leq \kappa \varepsilon^{4 m+2} r_{\varepsilon}^{-6 m+4-\delta},\left\|h_{j}^{(\dagger)}, k_{j}^{(\dagger)}\right\|_{\mathcal{B}_{j}} \leq \kappa \varepsilon^{2 m+4} r_{\varepsilon}^{2-4 m-\delta}\right\} .
\end{aligned}
$$

We call the functions in $\mathcal{B}(\kappa, \delta)$ pseudo-boundary data and will be used to parametrize solution of the Kcsc problem near a given "skeleton" solution built by hand to match some of the first orders of the metrics coming on the two sides of the gluing. 


\subsection{Kcsc metrics on the truncated base orbifold}

We start with a Kcsc orbifold $(M, \omega, g)$ with isolated singular points such that there is a subset of sungular points $\mathbf{p} \subset M$ whose elements have resolutions which are Ricci-flat ALE Kähler manifold. We want to find $F_{\mathbf{0}, \mathbf{b}, \mathbf{c}, \mathbf{h}, \mathbf{k}}^{\text {out }} \in C^{4, \alpha}\left(M_{r_{\varepsilon}}\right)$ such that,

$$
\omega_{\mathbf{0}, \mathbf{b}, \mathbf{c}, \mathbf{h}, \mathbf{k}}:=\omega+i \partial \bar{\partial} F_{\mathbf{0}, \mathbf{b}, \mathbf{c}, \mathbf{h}, \mathbf{k}}^{\text {out }}
$$

is a metric on $M_{r_{\varepsilon}}$ and its scalar curvature $s_{\omega_{\mathbf{0}, \mathbf{b}, \mathbf{c}, \mathbf{h}, \mathbf{k}}}$ is a small perturbation of the scalar curvature $s_{\omega}$ of the reference Kähler metric on $M$.

The function $F_{\mathbf{0}, \mathbf{b}, \mathbf{c}, \mathbf{h}, \mathbf{k}}^{\text {out }}$ consists of four blocks,

$$
F_{\mathbf{0}, \mathbf{b}, \mathbf{c}, \mathbf{h}, \mathbf{k}}^{\text {out }}:=-\varepsilon^{2 m} \mathbf{G}_{\mathbf{0}, \mathbf{b}, \mathbf{c}}+\mathbf{P}_{\mathbf{b}, \boldsymbol{\eta}}+\mathbf{H}_{\mathbf{h}, \mathbf{k}}^{\text {out }}+f_{\mathbf{0}, \mathbf{b}, \mathbf{c}, \mathbf{h}, \mathbf{k}}^{\text {out }}
$$

the skeleton $\varepsilon^{2 m} \mathbf{G}_{\mathbf{0}, \mathbf{b}, \mathbf{c}}$, extensions of pseudo-boundary data $\mathbf{H}_{\mathbf{h}, \mathbf{k}}^{\text {out }}$, transplanted potentials of $\eta_{j}$ 's $\mathbf{P}_{\mathbf{b}, \boldsymbol{\eta}}$ and a "small" correction term $f_{\mathbf{0}, \mathbf{b}, \mathbf{c}, \mathbf{h}, \mathbf{k}}^{\text {out }}$ that has to be determined. We want $F_{\mathbf{0}, \mathbf{b}, \mathbf{c}, \mathbf{h}, \mathbf{k}}^{\text {out }}$ be a small perturbation of $\omega$ and hence we can use the expansion in Proposition 3.1 to look for the equation that $f_{\mathbf{0}, \mathbf{b}, \mathbf{c}, \mathbf{h}, \mathbf{k}}^{\text {ou }}$ has to satisfy on $M_{r_{\varepsilon}}$. We have,

$$
\begin{aligned}
s_{\omega_{\mathbf{0}, \mathbf{b}, \mathbf{c}, \mathbf{h}, \mathbf{k}}}= & \mathbf{S}_{\omega}\left(-\varepsilon^{2 m} \mathbf{G}_{\mathbf{0}, \mathbf{b}, \mathbf{c}}+\mathbf{P}_{\mathbf{b}, \boldsymbol{\eta}}+\mathbf{H}_{\mathbf{h}, \mathbf{k}}^{\text {out }}+f\right) \\
= & s_{\omega}-\frac{1}{2} \varepsilon^{2 m} v_{\mathbf{0}, \mathbf{c}}-\frac{1}{2} \mathbb{L}_{\omega}\left[\mathbf{P}_{\mathbf{b}, \boldsymbol{\eta}}\right]-\frac{1}{2} \mathbb{L}_{\omega}\left[\mathbf{H}_{\mathbf{h}, \mathbf{k}}^{\text {out }}\right]-\frac{1}{2} \mathbb{L}_{\omega}[f] \\
& +\frac{1}{2} \mathbb{N}_{\omega}\left(-\varepsilon^{2 m} \mathbf{G}_{\mathbf{0}, \mathbf{b}, \mathbf{c}}+\mathbf{P}_{\mathbf{b}, \boldsymbol{\eta}}+\mathbf{H}_{\mathbf{h}, \mathbf{k}}^{\text {out }}+f\right),
\end{aligned}
$$

where in the second line we used the very definition of $\mathbf{G}_{\mathbf{0}, \mathbf{b}, \mathbf{c}}$. Rewriting the above equation in terms of the unknown $f$ we obtain,

$$
\begin{aligned}
\mathbb{L}_{\omega}[f]= & \left(2 s_{\omega}-\varepsilon^{2 m} v_{\mathbf{0}, \mathbf{c}}-2 s_{\omega_{0, \mathbf{b}, \mathbf{c}, \mathbf{h}, \mathbf{k}}}\right)-\mathbb{L}_{\omega}\left[\mathbf{P}_{\mathbf{b}, \boldsymbol{\eta}}\right]-\mathbb{L}_{\omega}\left[\mathbf{H}_{\mathbf{h}, \mathbf{k}}^{\text {out }}\right] \\
& +\mathbb{N}_{\omega}\left(-\varepsilon^{2 m} \mathbf{G}_{\mathbf{0}, \mathbf{b}, \mathbf{c}}+\mathbf{P}_{\mathbf{b}, \boldsymbol{\eta}}+\mathbf{H}_{\mathbf{h}, \mathbf{k}}^{\text {out }}+f\right) .
\end{aligned}
$$

The rest of this section is devoted to solve this equation.

Skeleton The skeleton is made of multi-poles fundamental solutions $\mathbf{G}_{\mathbf{0}, \mathbf{b}, \mathbf{c}}$ of $\mathbb{L}_{\omega}$ introduced in Sect. 3.2. These can be regarded as functions defined on $M_{\mathbf{p}}$ that are in ker $\mathbb{L}_{\omega}$ and blow up approaching points in $\mathbf{p}$. For this reason, the existence of a skeleton, is strictly related to balancing conditions (3.8) and (3.9) in Remark 3.1 with $\mathbf{a}=0$, namely,

$$
\begin{aligned}
\sum_{j=1}^{N} b_{j}\left(\Delta \varphi_{i}\right)\left(p_{j}\right)+\sum_{j=1}^{N} c_{j} \varphi_{i}\left(p_{j}\right) & =0 \\
\sum_{j=1}^{N} c_{j} & =v_{\mathbf{0}, \mathbf{c}} \operatorname{Vol}_{\omega}(M)
\end{aligned}
$$

so that,

$$
\mathbb{L}_{\omega}\left[\mathbf{G}_{\mathbf{0}, \mathbf{b}, \mathbf{c}}\right]+v_{\mathbf{0}, \mathbf{c}}=\sum_{j=1}^{N} b_{j} \Delta \delta_{p_{j}}+\sum_{j=1}^{N} c_{j} \delta_{p_{j}}, \quad \text { in } M .
$$


for a local description of the skeleton it is useful to keep in mind that, by Lemma 3.2, near points $p_{j}$ we have the expansion,

$$
\mathbf{G}_{\mathbf{0 , b}, \mathbf{c}} \sim \frac{b_{j}\left|\Gamma_{j}\right|}{2(m-1)\left|\mathbb{S}^{2 m-1}\right|} G_{\Delta}\left(p_{j}, z\right) .
$$

It is clear that the form,

$$
\begin{aligned}
& \omega+i \partial \bar{\partial}\left[-\varepsilon^{2 m} \mathbf{G}_{\mathbf{0}, \mathbf{b}, \mathbf{c}}+\left(\frac{b_{j}\left|\Gamma_{j}\right|}{2 c\left(\Gamma_{j}\right)(m-1)\left|\mathbb{S}^{2 m-1}\right|}\right)^{\frac{1}{m}} \varepsilon^{2} \chi_{j} \psi_{\eta_{j}}\right. \\
& \left.\quad \times\left(\left(\frac{2 c\left(\Gamma_{j}\right)(m-1)\left|\mathbb{S}^{2 m-1}\right|}{b_{j}\left|\Gamma_{j}\right|}\right)^{\frac{1}{m}} \frac{z}{\varepsilon}\right)\right]
\end{aligned}
$$

matches exactly at the highest order the form $\left(\frac{b_{j}\left|\Gamma_{j}\right|}{2 c\left(\Gamma_{j}\right)(m-1)\left|\mathbb{S}^{2 m-1}\right|}\right)^{\frac{1}{m}} \eta_{j}$, once we rescale (as we will in the final gluing) the model using the map,

$$
x=\left(\frac{2 c\left(\Gamma_{j}\right)(m-1)\left|\mathbb{S}^{2 m-1}\right|}{b_{j}\left|\Gamma_{j}\right|}\right)^{\frac{1}{m}} \frac{z}{\varepsilon}
$$

where the coefficient $c\left(\Gamma_{j}\right)$ is given by Proposition 2.3. It is then convenient, from now on, to set the following notation,

$$
B_{j}=\left(\frac{b_{j}\left|\Gamma_{j}\right|}{2 c\left(\Gamma_{j}\right)(m-1)\left|\mathbb{S}^{2 m-1}\right|}\right)^{\frac{1}{2 m}} .
$$

It will also be convenient to identify the right constants $C_{j}$ such that,

$$
\mathbb{L}_{\omega}\left(\mathbf{G}_{\mathbf{0}, \mathbf{b}, \mathbf{c}}-\sum_{j=1}^{N} c\left(\Gamma_{j}\right) B_{j}^{2 m} G_{\Delta}\left(p_{j}, z\right)+C_{j} G_{\Delta \Delta}\left(p_{j}, z\right)\right) \in C^{0, \alpha}(M) .
$$

By Lemma 3.3 one gets,

$$
C_{j}=\frac{\left|\Gamma_{j}\right|}{8(m-2)(m-1)}\left[2 c\left(\Gamma_{j}\right) B_{j}^{2 m} \frac{(m-1)\left|\mathbb{S}^{2 m-1}\right|}{m\left|\Gamma_{j}\right|} s_{\omega}\left(1+\frac{(m-1)^{2}}{(m+1)}\right)-c_{j}\right] .
$$

The highest blow-up terms of $G_{\Delta}, G_{\Delta \Delta}$ in $\mathbf{G}_{\mathbf{0}, \mathbf{b}, \mathbf{c}}$ i.e. terms exploding like $|z|^{2-2 m},|z|^{4-2 m}$ are the principal asymptotics of the family of Kcsc metrics $\omega_{\mathbf{0}, \mathbf{b}, \mathbf{c}, \mathbf{h}, \mathbf{k}}$. At the moment of data matching, the coefficients $B_{j}$ 's and $C_{j}$ 's will be "tuned" in such a way that, principal asymptotics of $\omega_{\mathbf{0}, \mathbf{b}, \mathbf{c}, \mathbf{h}, \mathbf{k}}$ on $M_{r_{\varepsilon}}$ will match exactly the "principal asymptotics" of $\varepsilon^{2} \eta_{\tilde{b}_{j}, \tilde{h}_{j}, \tilde{k}_{j}}$ 's on $X_{\Gamma_{j}, \frac{R_{\varepsilon}}{\tilde{b}_{j}}}$ 's. More precisely, under suitable rescalings, the $|z|^{2-2 m}$ terms of $\mathbf{G}_{\mathbf{0}, \mathbf{b}, \mathbf{c}}$ will match exactly the $|x|^{2-2 m}$ terms of the potentials at infinity of $\eta_{j}$ 's and also $|z|^{4-2 m}$ terms will match exactly the correction terms $|x|^{4-2 m}$ that pop up transplanting potential of $\omega$ on $X_{\Gamma_{j}}$. The justification for this procedure will come at the moment of data matching. indeed, when we will look at the metrics at the boundaries, it will be clear that the $\varepsilon$-growths of the principal asymptotics are the maximum among all terms constituting the family $\omega_{\mathbf{0}, \mathbf{b}, \mathbf{c}, \mathbf{h}, \mathbf{k}}$ and are in fact too large to be controlled by the extensions of pseudo-boundary data (introduced just below here). For general $\mathbf{b}, \mathbf{c}$ as in assumptions of Proposition 5.1 the data matching 
procedure becomes hence impossible. To overcome this difficulty we are forced to impose relations on $\mathbf{b}$, $\mathbf{c}$ with the tuning procedure, and in some sense we fix them, in order to have that the extensions of pseudo-boundary data control all the components of $\omega_{\mathbf{0}, \mathbf{b}, \mathbf{c}, \mathbf{h}, \mathbf{k}}$ not perfectly matched. The tuning procedure, although it could appear as a merely technical procedure, has strong geometric consequences indeed it yields to the right relations among the coefficients $b_{j}$ and $c_{j}$ giving us a precise asymptotics of the metrics produced.

Extensions of pseudo-boundary data Using the notion of euclidean outer biharmonic extensions of functions on the sphere we define for $(\mathbf{h}, \mathbf{k}) \in \mathcal{B}(\kappa, \delta)$,

$$
\mathbf{H}_{\mathbf{h}, \mathbf{k}}^{\text {out }}:=\sum_{j=1}^{N} \chi_{j} H_{h_{j}^{\text {out }}, k_{j}^{(\dagger)}}^{\left(\frac{z}{r_{\varepsilon}}\right) .}
$$

When we will look to this term at the boundary we will see that it has the second $\varepsilon$-growth after the principal asymptotics and it will become the highest $\varepsilon$-growth after the "tuning" of principal asymptotics. We will have, hence, that extensions of pseudo-boundary data dominate every other term with respect to $\varepsilon$-growth. Moreover thanks to the arbitrariness of $(\mathbf{h}, \mathbf{k})$, we can perform the Cauchy data matching procedure and glue the various metrics to a unique one.

Transplanted potentials As Székelyhidi does in [25,26], we bring to $M_{r_{\varepsilon}}$ the potentials of $\eta_{j}$ 's suitably rescaled and cut off in order to have better estimates through algebraic simplifications. Indeed, using the fact that $\eta_{j}$ 's are scalar flat we obtain some useful cancellations when compute the magnitude of the error we commit adding to $\omega$ "artificial" terms like the skeleton and the transplanted potentials. In $x$-coordinates on $X_{\Gamma_{j}}$ 's we have,

$$
\begin{aligned}
0 & \equiv \mathbf{S}_{\text {eucl }}\left(-c\left(\Gamma_{j}\right)|x|^{2-2 m}+\psi_{\eta_{j}}(x)\right) \\
& =-\frac{1}{2} \Delta^{2}\left[\psi_{\eta_{j}}(x)\right]+\frac{1}{2} \mathbb{N}_{\text {eucl }}\left(-c\left(\Gamma_{j}\right)|x|^{2-2 m}+\psi_{\eta_{j}}(x)\right),
\end{aligned}
$$

with $\psi_{\eta_{j}}$ 's potentials "at inifinity" of metrics $\eta_{j}$ 's defined in Sect. 2 Proposition 2.3 formula (2.5). With the rescaling,

$$
x=\frac{B_{j} z}{\varepsilon},
$$

where the coefficients $B_{j}$ 's are defined in formula (5.5), we consider the term,

$$
\mathbf{P}_{\mathbf{b}, \eta}:=\sum_{j=1}^{N} B_{j}^{2} \varepsilon^{2} \chi_{j} \psi_{\eta_{j}}\left(\frac{z}{B_{j} \varepsilon}\right) .
$$

We can rewrite identities (5.8) as follows,

$$
\begin{aligned}
0 & \equiv \mathbf{S}_{\text {eucl }}\left(-c\left(\Gamma_{j}\right) \varepsilon^{2 m} B^{2 m}|z|^{2-2 m}+\mathbf{P}_{\mathbf{b}, \boldsymbol{\eta}}\right) \\
& =-\frac{1}{2} \Delta^{2}\left[\mathbf{P}_{\mathbf{b}, \boldsymbol{\eta}}\right]+\frac{1}{2} \mathbb{N}_{\text {eucl }}\left(-c\left(\Gamma_{j}\right) \varepsilon^{2 m} B^{2 m}|z|^{2-2 m}+\mathbf{P}_{\mathbf{b}, \boldsymbol{\eta}}\right) .
\end{aligned}
$$

Unfortunately, since we are not in the euclidean setting, we have,

$$
-\frac{1}{2} \mathbb{L}_{\omega}\left[\mathbf{P}_{\mathbf{b}, \eta}\right]+\frac{1}{2} \mathbb{N}_{\omega}\left(-c\left(\Gamma_{j}\right) \varepsilon^{2 m} B^{2 m}|z|^{2-2 m}+\mathbf{P}_{\mathbf{b}, \boldsymbol{\eta}}\right) \neq 0 .
$$

and hence we produce an error that has to be corrected by the solution $f$ of the Eq. (5.4). The size of the solution $f$ grows as the error grows and we need $f$ to be small to be 
able to perform the Cauchy data matching procedure. So we want to minmize as much as possible this error. Here two facts come into play, the first is that on a small ball centered at $p_{j} \in \mathbf{p}$ the metric $\omega$ osculates with order two to the euclidean one and the second is that we substitute $c\left(\Gamma_{j}\right) \varepsilon^{2 m} B^{2 m}|z|^{2-2 m}$ with $\varepsilon^{2 m} \mathbf{G}_{\mathbf{0}, \mathbf{b}, \mathbf{c}}$ whose principal asymptotic is exactly $c\left(\Gamma_{j}\right) \varepsilon^{2 m} B^{2 m}|z|^{2-2 m}$. As we will see in the sequel we can use these two facts and relations (5.10) to produce sharp estimates for the error $\mathbf{S}_{\omega}\left(-\varepsilon^{2 m} \mathbf{G}_{\mathbf{0}, \mathbf{b}, \mathbf{c}}+\mathbf{P}_{\mathbf{b}, \boldsymbol{\eta}}\right)$ and verify that is sufficiently small to allow us to perform the Cauchy data matching procedure and hence conclude the gluing construction.

- Correction term It is the term that ensures the constancy of the scalar curvature of the metric $\omega_{\mathbf{0}, \mathbf{b}, \mathbf{c}, \mathbf{h}, \mathbf{k}}$ on $M_{r_{\varepsilon}}$ and it is a function $f_{\mathbf{0}, \mathbf{b}, \mathbf{c}, \mathbf{h}, \mathbf{k}}^{\text {out }} \in C_{\delta}^{4, \alpha}\left(M_{\mathbf{p}}\right) \oplus \mathcal{D}_{\mathbf{p}}(\mathbf{b}, \mathbf{c})$ if $m \geq 3$ and $f_{\mathbf{0}, \mathbf{b}, \mathbf{c}, \mathbf{h}, \mathbf{k}}^{\text {out }} \in C_{\delta}^{4, \alpha}\left(M_{\mathbf{p}}\right) \oplus \mathcal{E}_{\mathbf{p}} \oplus \mathcal{D}_{\mathbf{p}}(\mathbf{b}, \mathbf{c})$ if $m=2$, where the spaces $C_{\delta}^{4, \alpha}\left(M_{\mathbf{p}}\right) \oplus \mathcal{D}_{\mathbf{p}}(\mathbf{b}, \mathbf{c})$ and $C_{\delta}^{4, \alpha}\left(M_{\mathbf{p}}\right) \oplus \mathcal{E}_{\mathbf{p}} \oplus \mathcal{D}_{\mathbf{p}}(\mathbf{b}, \mathbf{c})$ are defined in Sect. 3.3 by formulas (3.11) and (3.11). As the notation suggests, the function $f_{\mathbf{0}, \mathbf{b}, \mathbf{c}, \mathbf{h}, \mathbf{k}}^{\text {out }}$ depends nonlinearly on $(\mathbf{h}, \mathbf{k})$ and $\mathbf{b}$ and we find it by solving a fixed point problem on a suitable closed and bounded subspace of $C_{\delta}^{4, \alpha}\left(M_{\mathbf{p}}\right) \oplus \mathcal{D}_{\mathbf{p}}(\mathbf{b}, \mathbf{c})$ if $m \geq 3$ and $\in C_{\delta}^{4, \alpha}\left(M_{\mathbf{p}}\right) \oplus \mathcal{E}_{\mathbf{p}} \oplus \mathcal{D}_{\mathbf{p}}(\mathbf{b}, \mathbf{c})$ if $m=2$.

Notation For the rest of the paper we will denote with $C$ a positive constant, that can vary from line to line, depending only on $\omega$ and $\eta_{j}$ 's.

We can now state the main proposition for the base space, whose proof at this point follows similar arguments as in [4,5] and is hence omitted:

Proposition 5.1 Let $(M, g, \omega)$ a Kcsc orbifold with isolated singularities and let $\mathbf{p}$ be the set of singular points with non trivial orbifold group that admit a Kähler Ricci flat resolution.

- Assume exist $\mathbf{b} \in\left(\mathbb{R}^{+}\right)^{N}$ and $\mathbf{c} \in \mathbb{R}^{N}$ such that,

$$
\begin{cases}\sum_{j=1}^{N} b_{j} \Delta_{\omega} \varphi_{i}\left(p_{j}\right)+c_{j} \varphi_{i}\left(p_{j}\right)=0 & i=1, \ldots, d \\ (\Theta(\mathbf{b}, \mathbf{c}))_{\substack{1 \leq i \leq d \\ 1 \leq j \leq N}} & \text { has rankd }\end{cases}
$$

where $(\Theta(\mathbf{b}, \mathbf{c}))_{\substack{1 \leq i \leq d \\ 1 \leq j \leq N}}$ is the matrix introduced in Sect. 3 formula (3.11). Let $\mathbf{G}_{\mathbf{0}, \mathbf{b}, \mathbf{c}}$ be the multi-poles solution of $\mathbb{L}_{\omega}$ constructed in Sect. 3 Remark 3.1.

- Let $\delta \in(4-2 m, 5-2 m)$. Given any $(\mathbf{h}, \mathbf{k}) \in \mathcal{B}(\kappa, \delta)$, where $\mathcal{B}(\kappa, \delta)$ is the space defined in formula (5.3), let $\mathbf{H}_{\mathbf{h}, \mathbf{k}}^{\text {out }}$ be the function defined in formula (5.7).

$$
\mathbf{H}_{\mathbf{h}, \mathbf{k}}^{\text {out }}:=\sum_{j=1}^{N} \chi_{j} H_{h_{j}^{(\dagger)}, k_{j}^{(\dagger)}}^{\text {out }}\left(\frac{z}{r_{\varepsilon}}\right) .
$$

- Let $\mathbf{P}_{\mathbf{b}, \eta}$ be the transplanted potentials defined in formula (5.9),

$$
\mathbf{P}_{\mathbf{b}, \eta}:=\sum_{j=1}^{N} B_{j}^{2} \varepsilon^{2} \chi_{j} \psi_{\eta_{j}}\left(\frac{z}{B_{j} \varepsilon}\right) .
$$

Then there exists $f_{\mathbf{0}, \mathbf{b}, \mathbf{c}, \mathbf{h}, \mathbf{k}}^{\text {out }} \in C_{\delta}^{4, \alpha}\left(M_{\mathbf{p}}\right) \oplus \mathcal{D}_{\mathbf{p}}(\mathbf{b}, \mathbf{c})$ if $m \geq 3$ and $f_{\mathbf{0}, \mathbf{b}, \mathbf{c}, \mathbf{h}, \mathbf{k}}^{\text {out }} \in C_{\delta}^{4, \alpha}\left(M_{\mathbf{p}}\right) \oplus$ $\mathcal{E}_{\mathbf{p}} \oplus \mathcal{D}_{\mathbf{p}}(\mathbf{b}, \mathbf{c})$ if $m=2$ such that,

$$
\omega_{\mathbf{0}, \mathbf{b}, \mathbf{c}, \mathbf{h}, \mathbf{k}}=\omega+i \partial \bar{\partial}\left(-\varepsilon^{2 m} \mathbf{G}_{\mathbf{0}, \mathbf{b}, \mathbf{c}}+\mathbf{P}_{\mathbf{b}, \boldsymbol{\eta}}+\mathbf{H}_{\mathbf{h}, \mathbf{k}}^{\text {out }}+f_{\mathbf{0}, \mathbf{b}, \mathbf{c}, \mathbf{h}, \mathbf{k}}^{\text {out }}\right) .
$$


is a Kcsc metric on $M_{r_{\varepsilon}}$ and the following estimates hold

$$
\begin{aligned}
&\left\|f_{\mathbf{0}, \mathbf{b}, \mathbf{c}, \mathbf{h}, \mathbf{k}}^{\text {out }}\right\|_{C_{\delta}^{4, \alpha}\left(M_{\mathbf{p}}\right) \oplus \mathcal{D}_{\mathbf{p}}(\mathbf{b}, \mathbf{c})} \leq C \varepsilon^{2 m+2} r_{\varepsilon}^{2-2 m-\delta} \quad \text { for } m \geq 3, \\
&\left\|f_{\mathbf{0}, \mathbf{b}, \mathbf{c}, \mathbf{h}, \mathbf{k}, \mathbf{k}}^{\text {out }}\right\|_{C_{\delta}^{4, \alpha}\left(M_{\mathbf{p}}\right) \oplus \mathcal{E}_{\mathbf{p}} \oplus \mathcal{D}_{\mathbf{p}}(\mathbf{b}, \mathbf{c})} \leq C \varepsilon^{6} r_{\varepsilon}^{-2-\delta} \quad \text { for } m=2 .
\end{aligned}
$$

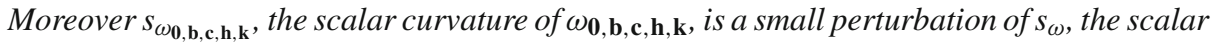
curvature of the background metric $\omega$ and we have,

$$
\left|s_{\omega_{\mathbf{0}, \mathbf{b}, \mathbf{c}, \mathbf{h}, \mathbf{k}}}-s_{\omega}\right| \leq C \varepsilon^{2 m} .
$$

\subsection{Kcsc metrics on the truncated model spaces}

We now want to perform on the model spaces $X_{\Gamma_{j}}$ 's a similar analysis as in the previous section.

Notation To keep notations as short as possible we drop the subscript $j$.

Our starting point is a Ricci-flat ALE Kähler manifold $\left(X_{\Gamma}, \eta, h\right)$ where we want to find $F_{\tilde{b}, \tilde{h}, \tilde{k}}^{i n} \in C^{4, \alpha}\left(X_{\Gamma, \frac{R_{\varepsilon}}{\tilde{b}}}\right)$ with $\tilde{b} \in \mathbb{R}^{+}$such that,

$$
\eta_{\tilde{b}, \tilde{h}, \tilde{k}}:=\tilde{b}^{2} \eta+i \partial \bar{\partial} F_{\tilde{b}, \tilde{h}, \tilde{k}}^{i n}
$$

is a metric on $X_{\frac{R_{\varepsilon}}{\bar{b}}}$ and,

$$
\mathbf{S}_{\tilde{b}^{2} \eta}\left(F_{\tilde{b}, \tilde{h}, \tilde{k}}^{i n}\right)=\varepsilon^{2}\left(s_{\omega}+\frac{1}{2} s_{\mathbf{0}, \mathbf{b}, \mathbf{c}, \mathbf{h}, \mathbf{k}}\right) .
$$

with $\mathbf{S}_{\tilde{b}^{2} \eta}$ the operator introduced in (2.1). The parameters $\tilde{b}, \tilde{h}, \tilde{k}$ will be chosen after the construction of the familiy of Kcsc metrics on $X_{\Gamma, \frac{R_{\varepsilon}}{\tilde{b}}}$, in particular $\tilde{b}$ will be chosen with a "manual tuning" of the principal asymptotics while $\tilde{h}, \tilde{k}$ with the Cauchy data matching procedure. The function $F_{\tilde{b}, \tilde{h}, \tilde{k}}^{i n}$ will be made of three blocks:

$$
F_{\tilde{b}, \tilde{h}, \tilde{k}}^{i n}:=\mathbf{P}_{\tilde{b}, \omega}+\mathbf{H}_{\tilde{h}, \tilde{k}}^{i n}+f_{\tilde{b}, \tilde{h}, \tilde{k}}^{i n} .
$$

$\mathbf{P}_{\tilde{b}, \omega}$ is the transplanted potential of $\omega$ that keeps the metric near to a Kcsc metric, $\mathbf{H}_{\tilde{h}, \tilde{k}}^{i n}$ is the extension of pseudo-boundary data that will allow us to perform the Cauchy data matching procedure and a small perturbation $f_{\tilde{b}, \tilde{h}, \tilde{k}}^{i n}$ that ensures the constancy of the scalar curvature. Since $F_{\tilde{b}, \tilde{h}, \tilde{k}}^{i n}$ has to be a small perturbation we can use the expansion in Proposition 3.1 to look for the equation that $f_{\tilde{b}, \tilde{h}, \tilde{k}}^{i n}$ has to satisfy and we have,

$$
\begin{aligned}
\varepsilon^{2} s_{\omega}+\frac{1}{2} \varepsilon^{2} s_{\mathbf{0}, \mathbf{b}, \mathbf{c}, \mathbf{h}, \mathbf{k}} & =\mathbf{S}_{\tilde{b}^{2} \eta}\left(\mathbf{P}_{\tilde{b}, \omega}+\mathbf{H}_{\tilde{h}, \tilde{k}}^{i n}+f\right) \\
& =\mathbf{S}_{\tilde{b}^{2} \eta}(0)-\frac{1}{2} \mathbb{L}_{\tilde{b}^{2} \eta}\left[\mathbf{P}_{\tilde{b}, \omega}+\mathbf{H}_{\tilde{h}, \tilde{k}}^{i n} f\right]+\frac{1}{2} \mathbb{N}_{\tilde{b}^{2} \eta}\left(\mathbf{P}_{\tilde{b}, \omega}+\mathbf{H}_{\tilde{h}, \tilde{k}}^{i n}+f\right)
\end{aligned}
$$

Remembering that $\mathbf{S}_{\tilde{b}^{2} \eta}(0)=0$ since $\eta$ is scalar flat and,

$$
\mathbb{L}_{\tilde{b}^{2} \eta}=\frac{1}{\tilde{b}^{4}} \Delta_{\eta}^{2},
$$


because $\eta$ is also Ricci-flat we can rewrite Eq. (5.11) in terms of the unknown $f$,

$$
\Delta_{\eta}^{2}[f]=-\varepsilon^{2} \tilde{b}^{4}\left(2 s_{\omega}+s_{\mathbf{0}, \mathbf{b}, \mathbf{c}, \mathbf{h}, \mathbf{k}}\right)-\Delta_{\eta}^{2}\left[\mathbf{P}_{\tilde{b}, \omega}+\mathbf{H}_{\tilde{h}, \tilde{k}}^{i n}\right]+\tilde{b}^{4} \mathbb{N}_{\tilde{b}^{2} \eta}\left(\mathbf{P}_{\tilde{b}, \omega}+\mathbf{H}_{\tilde{h}, \tilde{k}}^{i n}+f\right) .
$$

Transplanted potential As in $[25,26]$ we introduce the term $\mathbf{P}_{\tilde{b}, \omega}$ that is a suitable modification of the function $\psi_{\omega}$ defined in Proposition 2.1. We recall that $\psi_{\omega}$ satisfies,

$$
\mathbf{S}_{\text {eucl }}\left(\psi_{\omega}\right)=s_{\omega},
$$

and hence,

$$
s_{\omega}=-\frac{1}{2} \Delta^{2}\left[\psi_{\omega}\right]+\frac{1}{2} \mathbb{N}_{\text {eucl }}\left(\psi_{\omega}\right)
$$

in $z$ coordinates on a small ball. Once we perform the rescaling,

$$
z=\tilde{b} \varepsilon x
$$

we consider the function $\varepsilon^{-2} \psi_{\omega}(\tilde{b} \varepsilon x)$ and we have,

$$
\varepsilon^{2} s_{\omega}=-\frac{1}{2 \tilde{b}^{4}} \Delta^{2}\left[\frac{\psi_{\omega}(\tilde{b} \varepsilon x)}{\varepsilon^{2}}\right]+\frac{1}{2} \mathbb{N}_{\tilde{b}^{2} \cdot \text { eucl }}\left(\frac{\psi_{\omega}(\tilde{b} \varepsilon x)}{\varepsilon^{2}}\right)
$$

The aim of the transplanted potential is, hence, to cancel the term $\varepsilon^{2} s_{\omega}$ in Eq. (5.12). Unfortunately the metric associated to $\eta$ is not the euclidean one so remainder terms appear and the solution $f$ has to correct them, indeed we have,

$$
-\frac{1}{2 \tilde{b}^{4}} \Delta^{2}\left[\frac{\psi_{\omega}(\tilde{b} \varepsilon x)}{\varepsilon^{2}}\right]+\frac{1}{2} \mathbb{N}_{\tilde{b}^{2} \cdot \text { eucl }}\left(\frac{\psi_{\omega}(\tilde{b} \varepsilon x)}{\varepsilon^{2}}\right)=\varepsilon^{2} s_{\omega}+\text { remainder terms. }
$$

Remark 5.3 If the remainder terms of the equation above are too large, then the solution $f$ to the Eq. (5.12) becomes too large and it becomes impossible to perform the Cauchy data matching construction.

For simplicity we come back to the pre-rescaling expression of $\psi_{\omega}$ and we observe that by Lemma 2.1

$$
\begin{aligned}
\psi_{\omega} & =\sum_{k=0}^{+\infty} \Psi_{4+k}, \\
-\Delta^{2}\left[\Psi_{4}\right] & =2 s_{\omega}, \\
-\Delta^{2}\left[\Psi_{5}\right] & =0 .
\end{aligned}
$$

We have to correct the linear error committed by terms $\Psi_{4}, \Psi_{5}$ and hence we look for functions $W_{4}, W_{5}$ solutions of,

$$
\begin{aligned}
\Delta_{\eta}^{2}\left[\Psi_{4}+W_{4}\right] & =-2 s_{\omega} \\
\Delta_{\eta}^{2}\left[\Psi_{5}+W_{5}\right] & =0 .
\end{aligned}
$$

We point out that it will be crucial to obtain a description as explicit as possible of $W_{4}, W_{5}$. More precisely these corrections will be made of explicit terms and rapidly decaying terms. 
The first ones will impose constraints on the parameters of the balancing condition while the latter will be sufficiently small to be handled in the process of Cauchy data matching. The correction $W_{4}$, more precisely precisely one of its components, will give an extra constraint in the balancing condition and it is responsible for the requirement $c_{j}=s_{\omega} b_{j}$ in Theorem 1.1.

Notation For the rest of the section $\chi$ will denote a smooth cutoff function identically 0 on $X_{\Gamma, \frac{R_{0}}{3 \tilde{b}}}$ and identically 1 outside $X_{\Gamma, \frac{R_{0}}{2 \tilde{b}}}$.

Using Lemmas 4.1 and 2.1 it is easy to see that,

$$
\begin{aligned}
& \Delta_{\eta}^{2}\left[\chi \Psi_{4}\right]=-2 s_{\omega}+\left(\Phi_{2}+\Phi_{4}\right) \chi|x|^{-2 m}+\mathcal{O}\left(|x|^{-2-2 m}\right) \\
& \Delta_{\eta}^{2}\left[\chi \Psi_{5}\right]=\left(\Phi_{3}+\Phi_{5}\right) \chi|x|^{1-2 m}+\mathcal{O}\left(|x|^{-1-2 m}\right) .
\end{aligned}
$$

If we set

$$
\begin{aligned}
& u_{4}:= \begin{cases}\left(\frac{\Phi_{2}}{\Lambda_{2}^{2}}+\frac{\Phi_{4}}{\Lambda_{4}^{2}}\right) \chi|x|^{4-2 m} & \text { for } m \geq 3, \\
\left(\frac{\Phi_{2}}{\Lambda_{2}^{2}}+\frac{\Phi_{4}}{\Lambda_{4}^{2}}\right) \chi \log (|x|) & \text { for } m=2,\end{cases} \\
& u_{5}:=\left(\frac{\Phi_{3}}{\Lambda_{3}^{2}}+\frac{\Phi_{5}}{\Lambda_{5}^{2}}\right) \chi|x|^{5-2 m},
\end{aligned}
$$

for a suitable choice of $\Phi_{2}, \Phi_{4}, \Phi_{3}, \Phi_{5}$ eigenfunctions relative to the eigenvalues $\Lambda_{2}, \Lambda_{4}$, $\Lambda_{3}, \Lambda_{5}$ of $\Delta_{\mathbb{S} 2 m-1}$, then,

$$
\begin{aligned}
\Delta_{\eta}^{2}\left[\chi \Psi_{4}+u_{4}\right] & =-2 s_{\omega}+\mathcal{O}\left(|x|^{-2-2 m}\right), \\
\Delta_{\eta}^{2}\left[\chi \Psi_{5}+u_{5}\right] & =\mathcal{O}\left(|x|^{-1-2 m}\right) .
\end{aligned}
$$

Now we would like to find $v_{4} \in C_{\delta}^{4, \alpha}\left(X_{\Gamma}\right)$ with $\delta \in(2-2 m, 3-2 m)$ and $v_{5} \in C_{\delta}^{4, \alpha}\left(X_{\Gamma}\right)$ with $\delta \in(3-2 m, 4-2 m)$ such that,

$$
\begin{aligned}
& \Delta_{\eta}^{2}\left[\chi \Psi_{4}+u_{4}+v_{4}\right]=-2 s_{\omega} \\
& \Delta_{\eta}^{2}\left[\chi \Psi_{5}+u_{5}+v_{5}\right]=0 .
\end{aligned}
$$

Proposition 4.2 tells us that we can find such $v_{4}, v_{5}$ if and only if the integrals,

$$
\begin{aligned}
& \int_{X_{\Gamma}}\left(\Delta_{\eta}^{2}\left[\chi \Psi_{4}+u_{4}\right]+2 s_{\omega}\right) d \mu_{\eta} \\
& \int_{X_{\Gamma}} \Delta_{\eta}^{2}\left[\chi \Psi_{5}+u_{5}\right] d \mu_{\eta},
\end{aligned}
$$

vanish identically. To check whether those conditions are satisfied we have to compute the two integrals above. The crucial tool for the calculations is Lemma 2.4. We start computing integral (5.14). By means of divergence Theorem and Lemma 2.4 we can write,

$\int_{X_{\Gamma}}\left(\Delta_{\eta}^{2}\left[\chi \Psi_{4}+u_{4}\right]+2 s_{\omega}\right) d \mu_{\eta}=\lim _{\rho \rightarrow+\infty}\left[\int_{\partial X_{\Gamma, \rho}} \partial_{\nu} \Delta_{\eta}\left(\chi \Psi_{4}\right) d \mu_{\eta}+\frac{s_{\omega}\left|\mathbb{S}^{2 m-1}\right|}{m|\Gamma|} \rho^{2 m}\right]$,

with $v$ outward unit normal to the boundary. We point out that $u_{4}$ doesn't appear in the right hand side of the equation above because the boundary term produced by the integration by parts tends to zero as $\rho$ tends to infinity, and this is an immediate consequence of Lemma 4.1 
and the fact that $u_{4}$ has zero mean on every euclidean sphere. Then using Proposition 2.1 and Lemma 4.1,

$$
\begin{aligned}
\left.\partial_{\nu} \Delta_{\eta}\left[\Psi_{4}\right] d \mu_{\eta}\right|_{\partial X_{\Gamma, \rho}}= & {\left.\left[-\frac{s_{\omega}}{m} \rho^{2 m}-\frac{4 c_{X_{\Gamma}}(m-1)^{2} s_{\omega}}{m(m+1)}\right] d \mu_{0}\right|_{\mathbb{S}^{2 m-1} / \Gamma} } \\
& +\left.\left[\mathcal{O}(1)\left(\Phi_{2}+\Phi_{4}\right)+\mathcal{O}\left(\frac{1}{\rho}\right)\right] d \mu_{0}\right|_{\mathbb{S}^{2 m-1} / \Gamma},
\end{aligned}
$$

and integrating we obtain,

$$
\int_{X_{\Gamma}}\left(\Delta_{\eta}^{2}\left[\chi \Psi_{4}+u_{4}\right]+2 s_{\omega}\right) d \mu_{\eta}=-\frac{4 c_{X_{\Gamma}}(m-1)^{2}\left|\mathbb{S}^{2 m-1}\right| s_{\omega}}{m(m+1)|\Gamma|} .
$$

this shows that Eq. (5.13) cannot be solved in general for $v_{4} \in C_{\delta}^{4, \alpha}\left(X_{\Gamma}\right)$ with $\delta \in(2-2 m, 3-$ $2 m$ ). To overcome this difficulty we add an explicit function which belongs approximately to $\operatorname{ker}\left(\Delta_{\eta}^{2}\right)$, more precisely we can solve the equation,

$$
\begin{aligned}
& \Delta_{\eta}^{2}\left[\chi \Psi_{4}+u_{4}+\frac{c_{X_{\Gamma}}(m-1) s_{\omega}}{2(m-2) m(m+1)} \chi|x|^{4-2 m}+v_{4}\right]=-2 s_{\omega} \quad \text { for } m \geq 3 \\
& \Delta_{\eta}^{2}\left[\chi \Psi_{4}+u_{4}-\frac{c_{X_{\Gamma}} s_{\omega}}{6} \chi \log (|x|)+v_{4}\right]=-2 s_{\omega} \quad \text { for } m=2
\end{aligned}
$$

for $v_{4} \in C_{\delta}^{4, \alpha}(X)$ with $\delta \in(2-2 m, 3-2 m)$. In a completely analogous way we can compute integral (5.15) that vanishes identically and so we can solve the equation,

$$
\Delta_{\eta}^{2}\left[\chi \Psi_{5}+u_{5}+v_{5}\right]=0
$$

for $v_{5} \in C_{\delta}^{4, \alpha}(X)$ with $\delta \in(3-2 m, 4-2 m)$. Now we can write the explicit expression of $W_{4}$,

$$
W_{4}:=\left\{\begin{array}{ll}
\frac{c_{X_{\Gamma}}(m-1) s_{\omega}}{2(m-2) m(m+1)} \chi|x|^{4-2 m}+u_{4}+v_{4} & \text { for } m \geq 3, \\
-\frac{c_{X_{\Gamma}} s_{\omega}}{6} \chi \log (|x|)+u_{4}+v_{4} & \text { for } m=2 .
\end{array} .\right.
$$

The structure of the function $W_{4}$ deserves a word of comment, the function $v_{4}$ is what we call the rapidly decaying term, $u_{4}$ has a "critical" decaying rate but it has no radial components with respect to Fourier decomposition reative to $\Delta_{\mathbb{S}^{2 m-1}}$ and hence it will be handled by pseudo-boundary data in the Cauchy data matching, the remaning term is the one that will constrain the coefficients of the balancing condition.

Remark 5.4 The term $|x|^{4-2 m}$ (respectively $\log (|x|)$ ) in formula (5.16) plays a crucial role in our procedure, not only it is necessary for creating function on $X_{\Gamma}$ that rapidly decays $\Psi_{4}$ at infinity, but also influence the balancing condition. It forces, indeed, to require condition $c_{j}=s_{\omega} b_{j}$ in Theorem 1.1. In Sect. 6.1, we will see that, in order to be able to perform the data matching procedure, we will have to match perfectly (tuning procedure) the terms of the potential at inifinity of $\eta_{\tilde{b}, \tilde{h}, \tilde{k}}$ decaying as $|x|^{4-2 m}$ and $|x|^{2-2 m}$ with the principal asymptotics of the potential of $\omega_{\mathbf{0}, \mathbf{b}, \mathbf{c}, \mathbf{h}, \mathbf{k}}$ that are the terms exploding as $|z|^{2-2 m}$ and $|z|^{4-2 m}$. We will do this by making a specific choice for the parameters $\mathbf{b}$ and $\mathbf{c}$ and as a consequence we will get the key condition in Theorem 1.1. 
Contrarily to the case of $\Psi_{4}$ the correction of $\Psi_{5}$ is much easier indeed it is easy to see, using Lemma 4.1 and the fact that $u_{5}$ has no radial component with respect to the fourier decomposition relative to $\Delta_{\mathbb{S}^{2 m-1}}$, that,

$$
\lim _{\rho \rightarrow+\infty} \int_{\partial X_{\Gamma, \rho}} \partial_{\nu} \Delta_{\eta}\left[\chi \Psi_{5}+u_{5}\right]=0,
$$

and hence it is sufficient to apply Proposition 4.2 to find $v_{5}$. The function $W_{5}$ is then,

$$
W_{5}:=u_{5}+v_{5},
$$

and as for $W_{4}$ the function $v_{5}$ is a rapidly decaying term and $u_{4}$ has also a "critical" decaying rate but it has no radial components with respect to Fourier decomposition reative to $\Delta_{\mathbb{S}^{2 m-1}}$ and hence it will be handled by pseudo-boundary conditions in the Cauchy data matching. If we define,

$$
V:=\varepsilon^{2} \tilde{b}^{4} W_{4}+\varepsilon^{3} \tilde{b}^{5} W_{5}
$$

then we can define the transplanted potential $\mathbf{P}_{\tilde{b}, \omega}$ as the function in $C^{4, \alpha}\left(X_{\Gamma, \frac{R_{\varepsilon}}{\tilde{b}}}\right)$,

$$
\mathbf{P}_{\tilde{b}, \omega}:= \begin{cases}\frac{1}{\varepsilon^{2}} \chi \psi_{\omega}(\tilde{b} \varepsilon x)+V & \text { for } m \geq 3, \\ \frac{1}{\varepsilon^{2}} \chi \psi_{\omega}(\tilde{b} \varepsilon x)+V+C & \text { for } m=2 .\end{cases}
$$

where $C$ is the constant term in the expansion at $B_{2 r_{0}}(p) \backslash B_{r_{\varepsilon}}(p)$ of,

$$
F_{\mathbf{0}, \mathbf{b}, \mathbf{c}, \mathbf{h}, \mathbf{k}}^{\text {out }}=-\varepsilon^{2 m} \mathbf{G}_{\mathbf{0}, \mathbf{b}, \mathbf{c}}+\mathbf{P}_{\mathbf{b}, \boldsymbol{\eta}}+\mathbf{H}_{\mathbf{h}, \mathbf{k}}^{\text {out }}+f_{\mathbf{0}, \mathbf{b}, \mathbf{c}, \mathbf{h}, \mathbf{k}}^{\text {out }} .
$$

introduced in Proposition 5.1. As we will see in Sect. 6 the coefficient $\tilde{b}$ is very important and it will force the choice of particular values for the parameters $\mathbf{b}, \mathbf{c}$ we used on $M$ to construct $F_{\mathbf{0}, \mathbf{b}, \mathbf{c}, \mathbf{h}, \mathbf{k}}^{\text {out }}$, and in particular of the skeleton $\mathbf{G}_{\mathbf{0}, \mathbf{b}, \mathbf{c}}$.

Extensions of pseudo-boundary data Using euclidean inner biharmonic extensions of functions on the sphere we want to build a function on $X_{\Gamma}$ that is "almost" in the kernel of $\Delta_{\eta}^{2}$. We note that

$$
\begin{aligned}
\Delta_{\eta}^{2}\left[\chi|x|^{2}\right] & =\mathcal{O}\left(|x|^{-2-2 m}\right), \\
\Delta_{\eta}^{2}\left[\chi|x|^{2} \Phi_{2}\right] & =\mathcal{O}\left(|x|^{-2 m-2}\right), \\
\Delta_{\eta}^{2}\left[\chi|x|^{3} \Phi_{3}\right] & =\chi|x|^{-1-2 m} \Phi_{3}+\mathcal{O}\left(|x|^{-3-2 m}\right) .
\end{aligned}
$$

As for the transplanted potential we want to correct the functions on the left hand sides of equations in such a way they are in $\operatorname{ker}\left(\Delta_{\eta}^{2}\right)$. Precisely we want to solve the equations,

$$
\begin{aligned}
\Delta_{\eta}^{2}\left[\chi|x|^{2}+v^{(0)}\right] & =0, \\
\Delta_{\eta}^{2}\left[\chi|x|^{2} \Phi_{2}+v^{(2)}\right] & =0, \\
\Delta_{\eta}^{2}\left[\chi|x|^{3} \Phi_{3}+u^{(3)}+v^{(3)}\right] & =0 .
\end{aligned}
$$

with $v^{(0)}, v^{(2)}, v^{(3)} \in C_{\delta}^{4, \alpha}\left(X_{\Gamma}\right)$ for $\delta \in(2-2 m, 3-2 m)$ and,

$$
u^{(3)}:=\chi|x|^{3-2 m} \Phi_{3},
$$


for a suitable spherical harmonic $\Phi_{3}$. The existence of $v^{(0)}, v^{(2)}, v^{(3)}$ follows from Proposition 4.1, Lemma 4.2 and,

$$
\int_{X_{\Gamma}} \Delta_{\eta}^{2}\left[\chi|x|^{2}\right] d \mu_{\eta}=\int_{X_{\Gamma}} \Delta_{\eta}^{2}\left[\chi|x|^{2} \Phi_{2}\right] d \mu_{\eta}=\int_{X_{\Gamma}} \Delta_{\eta}^{2}\left[\chi|x|^{3} \Phi_{3}\right] d \mu_{\eta}=0,
$$

as one can easily check using exactly the same ideas exposed for the transplanted potential. We are ready to define the function $\mathbf{H}_{\tilde{h}, \tilde{k}}^{i n} \in C^{4, \alpha}\left(X_{\Gamma, \frac{R_{\varepsilon}}{\tilde{b}}}\right)$,

$$
\begin{aligned}
\mathbf{H}_{\tilde{h}, \tilde{k}}^{i n}:= & H_{\tilde{h}, \tilde{k}}^{i n}(0)+\chi\left(H_{\tilde{h}, \tilde{k}}^{i n}\left(\frac{\tilde{b} x}{R_{\varepsilon}}\right)-H_{\tilde{h}, \tilde{k}}^{i n}(0)\right)+\frac{\tilde{k}^{(0)} \tilde{b}^{2}}{4 m R_{\varepsilon}^{2}} v^{(0)} \\
& +\left(\tilde{h}^{(2)}-\frac{\tilde{k}^{(2)}}{4(m+2)}\right) \frac{\tilde{b}^{2} v^{(2)}}{R_{\varepsilon}^{2}}+\left(\tilde{h}^{(3)}-\frac{\tilde{k}^{(3)}}{4(m+3)}\right) \frac{\tilde{b}^{3}\left(u^{(3)}+v^{(3)}\right)}{R_{\varepsilon}^{3}} .
\end{aligned}
$$

Correction term It is the term that ensures the constancy of the scalar curvature of the metric $\eta_{\tilde{b}, \tilde{h}, \tilde{k}}$ on $X_{\Gamma, \frac{R_{\varepsilon}}{\tilde{b}}}$ and it is a function $f_{\tilde{b}, \tilde{h}, \tilde{k}}^{i n} \in C_{\delta}^{4, \alpha}\left(X_{\Gamma}\right)$ where the space $C_{\delta}^{4, \alpha}\left(X_{\Gamma}\right)$ is a weighted Hölder space defined in Sect. 4. As in the base orbifold, the function $f_{\tilde{b}, \tilde{h}, \tilde{k}}^{i n}$ depends nonlinearly on $(\tilde{h}, \tilde{k})$ and $\tilde{b}$ and we find it by solving a fixed point problem on a suitable closed and bounded subspace of $C_{\delta}^{4, \alpha}\left(X_{\Gamma}\right)$.

We are now ready to state the main result on the model spaces.

Proposition 5.2 Let $\left(X_{\Gamma}, h, \eta\right)$ an ALE Ricci-Flat Kähler resolution of an isolated quotient singularity.

- Let $\delta \in(4-2 m, 5-2 m)$. Given any $(\tilde{h}, \tilde{k}) \in \mathcal{B}$, such that $\left(\varepsilon^{2} \tilde{h}, \varepsilon^{2} \tilde{k}\right) \in \mathcal{B}(\kappa, \delta)$, where $\mathcal{B}(\kappa, \delta)$ is the space defined in formula (5.3), let $\mathbf{H}_{\tilde{h}, \tilde{k}}^{\text {in }}$ be the function defined in formula (5.18).

$$
\begin{aligned}
\mathbf{H}_{\tilde{h}, \tilde{k}}^{i n}: & =H_{\tilde{h}, \tilde{k}}^{i n}(0)+\chi\left(H_{\tilde{h}, \tilde{k}}^{i n}\left(\frac{\tilde{b} x}{R_{\varepsilon}}\right)-H_{\tilde{h}, \tilde{k}}^{i n}(0)\right)+\frac{\tilde{k}^{(0)} \tilde{b}^{2}}{4 m R_{\varepsilon}^{2}} v^{(0)} \\
& +\left(\tilde{h}^{(2)}-\frac{\tilde{k}^{(2)}}{4(m+2)}\right) \frac{\tilde{b}^{2} v^{(2)}}{R_{\varepsilon}^{2}}+\left(\tilde{h}^{(3)}-\frac{\tilde{k}^{(3)}}{4(m+3)}\right) \frac{\tilde{b}^{3}\left(u^{(3)}+v^{(3)}\right)}{R_{\varepsilon}^{3}} .
\end{aligned}
$$

- Let $\mathbf{P}_{\tilde{b}, \omega}$ be the transplanted potential defined in formula (5.17)

$$
\mathbf{P}_{\tilde{b}, \omega}:= \begin{cases}\frac{1}{\varepsilon^{2}} \chi \psi_{\omega}(\tilde{b} \varepsilon x)+V & \text { for } m \geq 3 \\ \frac{1}{\varepsilon^{2}} \chi \psi_{\omega}(\tilde{b} \varepsilon x)+V+C & \text { for } m=2 .\end{cases}
$$

Then there is $f_{\tilde{b}, \tilde{h}, \tilde{k}}^{i n} \in C_{\delta}^{4, \alpha}\left(X_{\Gamma}\right)$ such that

$$
\eta_{\tilde{b}, \tilde{h}, \tilde{k}}=\tilde{b}^{2} \eta+i \partial \bar{\partial}\left(\mathbf{P}_{\tilde{b}, \omega}+\mathbf{H}_{\tilde{h}, \tilde{k}}^{i n}+f_{\tilde{b}, \tilde{h}, \tilde{k}}^{i n}\right),
$$

is a Kcsc metric on $X_{\Gamma, \frac{R_{\varepsilon}}{\bar{b}}}$ and the following estimates hold.

$$
\left\|f_{\tilde{b}, \tilde{h}, \tilde{k}}^{i n}\right\|_{C_{\delta}^{4, \alpha}\left(X_{\Gamma}\right)} \leq C(\kappa) \varepsilon^{2 m+4} r_{\varepsilon}^{-4 m-\delta} R_{\varepsilon}^{-2},
$$


with $C(\kappa) \in \mathbb{R}^{+}$depending only on $\omega$ and $\eta_{j}$ 's and $\kappa$ the constant appearing in the definition

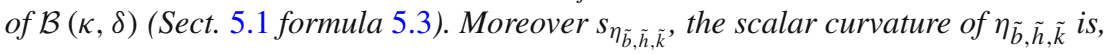

$$
s_{\eta_{\tilde{b}, \tilde{h}, \tilde{k}}}=s_{\omega_{\mathbf{0}, \mathbf{b}, \mathbf{h}, \mathbf{k}}}=s_{\omega}+\frac{1}{2} s_{\mathbf{0}, \mathbf{b}, \mathbf{h}, \mathbf{k}} \cdot
$$

Again, the proof follows from similar arguments as in [4,5] repeatedly using the Ricci-flat condition.

\section{Data matching}

Now that we have the families of metrics on the base orbifold and on model spaces we want to glue them. To perform the data matching construction we will rescale all functions involved in such a way that functions on $X_{\Gamma_{j}}$ are functions on the annulus $\overline{B_{1}} \backslash B_{\frac{1}{2}}$ and functions on $M$ are functions on the annulus $\overline{B_{2}} \backslash B_{1}$. The main technical tool we will use in this section is the "Dirichet to Neumann" map for euclidean biharmonic extensions that we introduce with the following Theorem whose proof can be found in [4, Lemma 6.3].

Theorem 6.1 The map

$$
\begin{aligned}
& \mathcal{P}: C^{4, \alpha}\left(\mathbb{S}^{2 m-1}\right) \times C^{2, \alpha}\left(\mathbb{S}^{2 m-1}\right) \rightarrow C^{3, \alpha}\left(\mathbb{S}^{2 m-1}\right) \times C^{1, \alpha}\left(\mathbb{S}^{2 m-1}\right) \\
& \mathcal{P}(h, k)=\left(\partial_{|w|}\left(H_{h, k}^{\text {out }}-H_{h, k}^{\text {in }}\right), \partial_{|w|} \Delta\left(H_{h, k}^{\text {out }}-H_{h, k}^{\text {in }}\right)\right)
\end{aligned}
$$

is an isomorphism of Banach spaces with inverse $\mathcal{Q}$.

Proof of Theorem 1.1 We carry on the proof for the case $m \geq 3$, for $m=2$ it is identical. Let $\mathcal{V}_{j, \mathbf{0}, \mathbf{b}, \mathbf{c}, \mathbf{h}, \mathbf{k}}^{\text {unt }}$ be Kähler potential of $\omega_{\mathbf{0}, \mathbf{b}, \mathbf{c}, \mathbf{h}, \mathbf{k}}$ at the annulus $\overline{B_{2 r_{\varepsilon}}\left(p_{j}\right)} \backslash B_{r_{\varepsilon}}\left(p_{j}\right)$ under the homothety

$$
z=r_{\varepsilon} w
$$

We have then the expansion

$$
\begin{aligned}
\mathcal{V}_{j, \mathbf{0}, \mathbf{b}, \mathbf{c}, \mathbf{h}, \mathbf{k}}^{\text {out }}= & \frac{r_{\varepsilon}^{2}}{2}|w|^{2}+\psi_{\omega}\left(r_{\varepsilon} w\right)+\varepsilon^{2} \psi_{\eta_{j}}\left(\frac{r_{\varepsilon} w}{B_{j} \varepsilon}\right) \\
& +\left(1-\frac{\left(f_{\mathbf{0}, \mathbf{b}, \mathbf{c}, \mathbf{h}, \mathbf{k}}^{\text {out }}\right)^{j}}{\varepsilon^{2 m}}\right)\left(-c_{X_{\Gamma_{j}}} B_{j}^{2 m} \varepsilon^{2 m} r_{\varepsilon}^{2-2 m}|w|^{2-2 m}+C_{j} \varepsilon^{2 m} r_{\varepsilon}^{4-2 m}|w|^{4-2 m}\right) \\
& +H_{h_{j}^{\text {out }}, k_{j}^{(\dagger)}}^{(\dagger)} \\
& -\left[\varepsilon^{2 m} \mathbf{G}_{\mathbf{0}, \mathbf{b}, \mathbf{c}}-c\left(\Gamma_{j}\right) B_{j}^{2 m} \varepsilon^{2 m} r_{\varepsilon}^{2-2 m}|w|^{2-2 m}+C_{j} \varepsilon^{2 m} r_{\varepsilon}^{4-2 m}|w|^{4-2 m}\right] \\
& +\left[f_{\mathbf{0}, \mathbf{b}, \mathbf{c}, \mathbf{h}, \mathbf{k}}^{\text {out }}+\left(f_{\mathbf{0}, \mathbf{b}, \mathbf{c}, \mathbf{h}, \mathbf{k}}^{\text {out }}\right)^{j}\left(c_{X_{\Gamma_{j}}} B_{j}^{2 m} r_{\varepsilon}^{2-2 m}|w|^{2-2 m}-C_{j} r_{\varepsilon}^{4-2 m}|w|^{4-2 m}\right)\right] .
\end{aligned}
$$

For the sake of notation we set,

$$
\begin{aligned}
\mathcal{R}_{j}^{\text {out }}:= & -\left[\varepsilon^{2 m} \mathbf{G}_{\mathbf{0}, \mathbf{b}, \mathbf{c}}-c_{X_{\Gamma_{j}}} B_{j}^{2 m} \varepsilon^{2 m} r_{\varepsilon}^{2-2 m}|w|^{2-2 m}+C_{j} \varepsilon^{2 m} r_{\varepsilon}^{4-2 m}|w|^{4-2 m}\right] \\
& +\left[f_{\mathbf{0}, \mathbf{b}, \mathbf{c}, \mathbf{h}, \mathbf{k}}^{\text {out }}+\left(f_{\mathbf{0}, \mathbf{b}, \mathbf{c}, \mathbf{h}, \mathbf{k}}^{\text {out }}\right)^{j}\left(c_{X_{\Gamma_{j}}} B_{j}^{2 m} r_{\varepsilon}^{2-2 m}|w|^{2-2 m}-C_{j} r_{\varepsilon}^{4-2 m}|w|^{4-2 m}\right)\right] .
\end{aligned}
$$


We recall that, using notations of Theorem 3.4, $f_{\mathbf{0}, \mathbf{b}, \mathbf{c}, \mathbf{h}, \mathbf{k}}^{\text {out }} \in C_{\delta}^{4, \alpha}\left(M_{\mathbf{p}}\right) \oplus \mathcal{D}(\mathbf{b}, \mathbf{c})$ and we have,

$$
f_{\mathbf{0}, \mathbf{b}, \mathbf{c}, \mathbf{h}, \mathbf{k}}^{\text {out }}=\tilde{f}_{\mathbf{0}, \mathbf{b}, \mathbf{c}, \mathbf{h}, \mathbf{k}}^{\text {out }}+\sum_{j=1}^{N}\left(f_{\mathbf{0}, \mathbf{b}, \mathbf{c}, \mathbf{h}, \mathbf{k}}^{\text {out }}\right)^{j} W_{\mathbf{b}, \mathbf{c}}^{j}
$$

with $\tilde{f}_{\mathbf{0}, \mathbf{b}, \mathbf{c}, \mathbf{h}, \mathbf{k}}^{\text {out }} \in C_{\delta}^{4, \alpha}\left(M_{\mathbf{p}}\right)$ for $\delta \in(4-2 m, 5-2 m)$ and the numbers $\left(f_{\mathbf{0}, \mathbf{b}, \mathbf{c}, \mathbf{h}, \mathbf{k}}^{\text {out }}\right)^{j}$ 's are the coefficients of the deficiency components of $f_{\mathbf{0}, \mathbf{b}, \mathbf{c}, \mathbf{h}, \mathbf{k}}^{\text {out }}$. In writing the expansion of $\mathcal{V}_{j, \mathbf{0}, \mathbf{b}, \mathbf{c}, \mathbf{h}, \mathbf{k}}^{\text {out }}$, precisely in the second and fourth lines, we used the only principal asymptotics of $\left(f_{\mathbf{0}, \mathbf{b}, \mathbf{c}, \mathbf{h}, \mathbf{k}}^{\text {out }}\right)^{j} W_{\mathbf{b}, \mathbf{c}}^{j}$ exposed in formula (3.10) while the remaining part falls in the remainder term $\mathcal{R}_{j}^{\text {out }}$.

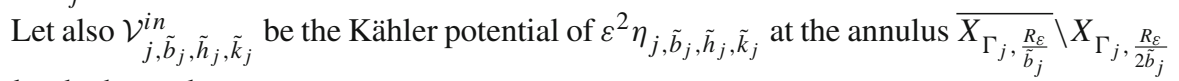
under the homothety,

$$
x=\frac{R_{\varepsilon} w}{\tilde{b}_{j}} .
$$

We have the expansion

$$
\begin{aligned}
\mathcal{V}_{j, \tilde{b}_{j}, \tilde{h}_{j}, \tilde{k}_{j}}^{i n} & \frac{\varepsilon^{2} R_{\varepsilon}^{2}}{2}|w|^{2}+\varepsilon^{2} B^{2} \psi_{\eta_{j}}\left(\frac{R_{\varepsilon} w}{B_{j}}\right)+\psi_{\omega}\left(\varepsilon R_{\varepsilon} w\right) \\
& -c\left(\Gamma_{j}\right) \tilde{b}_{j}^{2 m} \varepsilon^{2} R_{\varepsilon}^{2-2 m}|w|^{2-2 m}+\frac{c_{X_{\Gamma_{j}}}(m-1) s_{\omega} \tilde{b}^{4} \varepsilon^{4} R_{\varepsilon}^{4-2 m}}{2(m-2) m(m+1)}|w|^{4-2 m} \\
& \left.+H_{\varepsilon^{2} \tilde{h}_{j}, \varepsilon^{2} \tilde{k}_{j}}^{i n}\right] \\
& +\left[\varepsilon^{2} \mathbf{P}_{\tilde{b}_{,} \omega}-\psi_{\omega}\left(\varepsilon R_{\varepsilon} w\right)-\frac{c_{X_{\Gamma_{j}}}(m-1) s_{\omega} \tilde{b}^{4} \varepsilon^{4} R_{\varepsilon}^{4-2 m}}{2(m-2) m(m+1)}|w|^{4-2 m}\right] \\
& +\left[\varepsilon^{2} \tilde{b}_{j}^{2} \psi_{\eta_{j}}\left(\frac{R_{\varepsilon} w}{\tilde{b}_{j}}\right)-\varepsilon^{2} B_{j}^{2} \psi_{\eta_{j}}\left(\frac{R_{\varepsilon} w}{B_{j}}\right)\right]+\left[\mathbf{H}_{\varepsilon^{2} \tilde{h}_{j}, \varepsilon^{2} \tilde{k}_{j}}-H_{\varepsilon^{2} \tilde{h}_{j}, \varepsilon^{2} \tilde{k}_{j}}^{i n}\right] \\
& +\varepsilon^{2} f_{\tilde{b}_{j}, \tilde{h}_{j}, \tilde{k}_{j}}^{i n} \cdot
\end{aligned}
$$

For the sake of notation we set,

$$
\begin{aligned}
\mathcal{R}_{j}^{i n}:= & {\left[\varepsilon^{2} \mathbf{P}_{\tilde{b}, \omega}-\psi_{\omega}\left(\varepsilon R_{\varepsilon} w\right)-\frac{c_{X_{\Gamma_{j}}}(m-1) s_{\omega} \tilde{b}^{4} \varepsilon^{4} R_{\varepsilon}^{4-2 m}}{2(m-2) m(m+1)}|w|^{4-2 m}\right] } \\
& +\left[\varepsilon^{2} \tilde{b}_{j}^{2} \psi_{\eta_{j}}\left(\frac{R_{\varepsilon} w}{\tilde{b}_{j}}\right)-\varepsilon^{2} B_{j}^{2} \psi_{\eta_{j}}\left(\frac{R_{\varepsilon} w}{B_{j}}\right)\right]+\left[\mathbf{H}_{\varepsilon^{2} \tilde{h}_{j}, \varepsilon^{2} \tilde{k}_{j}}^{i n}-H_{\varepsilon^{2} \tilde{h}_{j}, \varepsilon^{2} \tilde{k}_{j}}^{i n}\right] \\
& +\varepsilon^{2} f_{\tilde{b}_{j}, \tilde{h}_{j}, \tilde{k}_{j}}^{i n}
\end{aligned}
$$

We want to find $\mathbf{b}, \mathbf{c}, \tilde{\mathbf{b}}, \mathbf{h}, \mathbf{k}, \tilde{\mathbf{h}}, \tilde{\mathbf{k}}$ such that the functions,

$$
\mathcal{V}_{j}:= \begin{cases}\mathcal{V}_{j, \tilde{b}_{j}, \tilde{h}_{j}, \tilde{k}_{j}}^{\text {in }} & \text { on } B_{1} \backslash B_{\frac{1}{2}} \\ \mathcal{V}_{j, \mathbf{0}, \mathbf{b}, \mathbf{c}, \mathbf{h}, \mathbf{k}}^{\text {out }} & \text { on } \overline{B_{2}} \backslash B_{1}\end{cases}
$$


are smooth on $\overline{B_{2}} \backslash B_{\frac{1}{2}}$ for every $j=1, \ldots, N$. We have written the expansions of $\mathcal{V}_{j, \mathbf{0}, \mathbf{b}, \mathbf{c}, \mathbf{h}, \mathbf{k}}^{\text {out }}$ 's

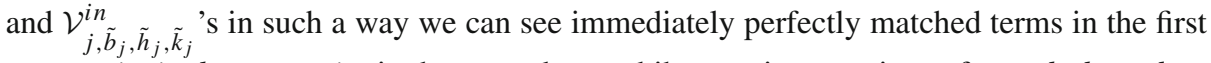
rows, principal asymptotics in the second rows, biharmonic extensions of pseudo-boundary data in the third rows, and "remainder" terms.

\subsection{Tuning procedure}

We would like to have that also the principal asymptotics match perfectly and biharmonic extensions of pseudo-boundary data dominate all the "remainder terms" in $\varepsilon$-growth. Moreover we need to recover a degree of freedom in biharmonic extensions since we have have taken meanless functions $\mathbf{h}^{(\dagger)}, \mathbf{k}^{(\dagger)}$ as parameters. To overcome these problems we have to perform a "tuning" of the principal asymptotics i.e. we have to set,

$$
\begin{aligned}
c_{X_{\Gamma_{j}}} \tilde{b}_{j}^{2 m} \varepsilon^{2} R_{\varepsilon}^{2-2 m}|w|^{2-2 m}= & \left(1-\frac{\left(f_{\mathbf{0}, \mathbf{b}, \mathbf{c}, \mathbf{h}, \mathbf{k}}^{\text {out }}\right)^{j}}{\varepsilon^{2 m}}\right) c_{X_{\Gamma_{j}}} B_{j}^{2 m} \varepsilon^{2 m} r_{\varepsilon}^{2-2 m}|w|^{2-2 m} \\
& +\left(h_{j}^{(0)}+\frac{k_{j}^{(0)}}{4 m-8}\right)|w|^{2-2 m} \\
\frac{c_{X_{\Gamma_{j}}}(m-1) s_{\omega} \tilde{b}^{2 m} \varepsilon^{4} R_{\varepsilon}^{4-2 m}|w|^{4-2 m}=}{2(m-2) m(m+1)} & -\left(1-\frac{\left(f_{\mathbf{0}, \mathbf{b}, \mathbf{c}, \mathbf{h}, \mathbf{k}}^{\text {out }}\right)^{j}}{\varepsilon^{2 m}}\right) C_{j} \varepsilon^{2 m} r_{\varepsilon}^{4-2 m}|w|^{4-2 m} \\
& -\frac{k_{j}^{(0)}|w|^{4-2 m} .}{4 m-8} .
\end{aligned}
$$

With the specialization above we regain the means of functions $h_{j}$ and $k_{j}$. In fact, as we can see from formula (5.1), choosing meanless functions we were missing exactly the radial terms in the Fourier expansion of $H_{h, k}^{\text {out }}$ that incidentally have exactly the same growth of the principal asymptotics. So perturbing a bit the coefficients $b_{j}$ 's we can recover these missing asymptotics in the biharmonic extensions but Eq. (6.1) imposes us to set the value of parameter $\tilde{b}_{j}$. Moreover, we point out that once we have set the value of $\tilde{b}_{j}$ the Eq. (6.2) imposes us to choose a particular value for the parameter $c_{j}$ and hence we see, as anticipated in Sect. 5.3, how the nonlinear analysis on $X_{\Gamma_{j}}$ 's constrains the parameters of balancing condition. We recall that coefficients $B_{j}$ and $C_{j}$ are defined in Sect. 5.2 respectively by Eqs. (5.5) and (5.6). Conditions above force us to set:

$$
\begin{aligned}
\tilde{b}_{j}^{2 m} & =B_{j}^{2 m}\left(1-\frac{\left(f_{\mathbf{0}, \mathbf{b}, \mathbf{c}, \mathbf{h}, \mathbf{k}}^{\text {out }}\right)^{j}}{\varepsilon^{2 m}}\right)+\frac{1}{c_{X_{\Gamma_{j}}}}\left(h_{j}^{(0)}+\frac{k_{j}^{(0)}}{4 m-8}\right) \frac{r_{\varepsilon}^{2 m-2}}{\varepsilon^{2 m}} \\
C_{j} & =-\frac{1}{2(m-2)\left(\varepsilon^{2 m}-\left(f_{\mathbf{0}, \mathbf{b}, \mathbf{c}, \mathbf{h}, \mathbf{k}}^{\text {out }}\right)^{j}\right)}\left(\frac{c_{X_{\Gamma_{j}}}(m-1) s_{\omega} \tilde{b}_{j}^{2 m} \varepsilon^{4} R_{\varepsilon}^{4-2 m}}{m(m+1)}+k_{j}^{(0)}\right)
\end{aligned}
$$

and hence we must set

$$
c_{j}=s_{\omega} b_{j}
$$


Remark 6.1 At this point, the presence of $|x|^{4-2 m}$ term in the correction $W_{4}$, introduced in Sect. 5.3 formula (5.16), shows its effects. That term indeed, introduced as a technical tool for obtaining better estimates, puts now strong geometric constraints on our construction defining the correct form of non degeneracy condition and balancing condition forcing us to impose Eq. (6.2) and giving as consequence relations (6.3), (6.4) and the key condition (6.5).

We can see also that,

$$
\left\|\left(\mathcal{R}_{j}^{\text {out }}\right)^{(0)}\right\|_{C^{4, \alpha}\left(\overline{B_{2}} \backslash B_{1}\right)},\left\|\left(\mathcal{R}_{j}^{\text {out }}\right)^{(0)}\right\|_{C^{4, \alpha}\left(\overline{B_{1} \backslash B_{\frac{1}{2}}}\right)}=o\left(\varepsilon^{4 m+2} r_{\varepsilon}^{-6 m+4-\delta}\right)
$$

and,

$$
\left\|\left(\mathcal{R}_{j}^{\text {out }}\right)^{(\dagger)}\right\|_{C^{4, \alpha}\left(\overline{B_{2}} \backslash B_{1}\right)},\left\|\left(\mathcal{R}_{j}^{\text {out }}\right)^{(\dagger)}\right\|_{C^{4, \alpha}\left(\overline{B_{1} \backslash B_{\frac{1}{2}}}\right)}=o\left(\varepsilon^{2 m+4} r_{\varepsilon}^{2-4 m-\delta}\right),
$$

therefore the biharmonic extensions dominate all remainder terms in $\varepsilon$-growth indeed,

$$
\left|\mathbf{h}^{(0)}\right|+\left|\mathbf{k}^{(0)}\right|=\mathcal{O}\left(\varepsilon^{4 m+2} r_{\varepsilon}^{-6 m+4-\delta}\right) \quad \text { and } \quad\left\|\mathbf{h}^{(\dagger)}, \mathbf{k}^{(\dagger)}\right\|_{\mathcal{B}(\kappa, \delta)}=\mathcal{O}\left(\varepsilon^{2 m+4} r_{\varepsilon}^{2-4 m-\delta}\right) .
$$

\subsection{Cauchy data matching procedure}

Now we want to find the correct parameters such that at $\mathbb{S}^{2 m-1}$ there is a $C^{3}$ matching

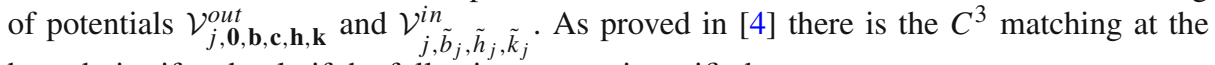
boundaries if and only if the following system is verified,

$$
\left(\Sigma_{j}\right):\left\{\begin{array}{l}
\mathcal{V}_{j, \mathbf{0}, \mathbf{b}, \mathbf{c}, \mathbf{h}, \mathbf{k}}^{\text {out }}=\mathcal{V}_{j, \tilde{b}_{j}, \tilde{h}_{j}, \tilde{k}_{j}}^{\text {in }} \\
\partial_{|w|}\left[\mathcal{V}_{j, \mathbf{0}, \mathbf{b}, \mathbf{c}, \mathbf{h}, \mathbf{k}}^{\text {out }}\right]=\partial_{|w|}\left[\mathcal{V}_{j, \tilde{b}_{j}, \tilde{h}_{j}, \tilde{k}_{j}}^{\text {in }}\right] \\
\Delta\left[\mathcal{V}_{j, \mathbf{0}, \mathbf{b}, \mathbf{c}, \mathbf{h}, \mathbf{k}}^{\text {out }}\right]=\Delta\left[\mathcal{V}_{j, \tilde{b}_{j}, \tilde{h}_{j}, \tilde{k}_{j}}^{\text {in }}\right] \\
\partial_{|w|} \Delta\left[\mathcal{V}_{j, \mathbf{0}, \mathbf{b}, \mathbf{c}, \mathbf{h}, \mathbf{k}}^{\text {out }}\right]=\partial_{|w|} \Delta\left[\mathcal{V}_{j, \tilde{b}_{j}, \tilde{h}_{j}, \tilde{k}_{j}}^{\text {in }}\right]
\end{array}\right.
$$

After choices (6.3),(6.4), (6.5) and some algebraic manipulations, systems $\left(\Sigma_{j}\right)$ become,

$$
\left(\Sigma_{j}\right):\left\{\begin{array}{l}
\varepsilon^{2} \tilde{h}_{j}=h_{j}-\xi_{j} \\
\varepsilon^{2} \tilde{k}_{j}=k_{j}-\Delta\left[\xi_{j}\right] \\
\partial_{|w|}\left[H_{h_{j}, k_{j}}^{\text {out }}-H_{h_{j}, k_{j}}^{\text {in }}\right]=\partial_{|w|}\left[\xi_{j}-H_{\xi_{j}, \Delta \xi_{j}}^{\text {in }}\right] \\
\partial_{|w|} \Delta\left[H_{h_{j}, k_{j}}^{\text {out }}-H_{h_{j}, k_{j}}^{I}\right]=\partial_{|w|} \Delta\left[\xi_{j}-H_{\xi_{j}, \Delta \xi_{j}}^{\text {in }}\right]
\end{array}\right.
$$

with $\xi_{j}$ a function depending linearly $\mathcal{R}_{j}^{\text {out }}$ and $\mathcal{R}_{j}^{i n}$. Using Theorem 6.1 we define the operators,

$\mathcal{S}_{j}\left(\varepsilon^{2} \tilde{h}_{j}, \varepsilon^{2} \tilde{k}_{j}, h_{j}, k_{j}\right):=\left(h_{j}-\xi_{j}, k_{j}-\Delta \xi_{j}, \mathcal{Q}\left[\partial_{|w|}\left(\xi_{j}-H_{\xi_{j}, \Delta \xi_{j}}^{i n}\right), \partial_{|w|} \Delta\left(\xi_{j}-H_{\xi_{j}, \Delta \xi_{j}}^{i n}\right)\right]\right)$

and then the operator $\mathcal{S}: \mathcal{B}(\kappa, \delta)^{2} \rightarrow \mathcal{B}^{2}$,

$$
\mathcal{S}:=\left(\mathcal{S}_{1}, \ldots, \mathcal{S}_{N}\right) .
$$


Note also that biharmonic extensions, seen as operators,

$$
H_{\cdot, \cdot,}^{\text {out }}, H_{\cdot, \cdot}^{\text {in }}: C^{4, \alpha}\left(\mathbb{S}^{2 m-1}\right) \times C^{2, \alpha}\left(\mathbb{S}^{2 m-1}\right) \rightarrow C^{4, \alpha}\left(\mathbb{S}^{2 m-1}\right)
$$

and the operator,

$$
\mathcal{Q}: C^{3, \alpha}\left(\mathbb{S}^{2 m-1}\right) \times C^{1, \alpha}\left(\mathbb{S}^{2 m-1}\right) \rightarrow C^{4, \alpha}\left(\mathbb{S}^{2 m-1}\right) \times C^{2, \alpha}\left(\mathbb{S}^{2 m-1}\right)
$$

defined in Proposition 6.1, preserve eigenspaces of $\Delta_{\mathbb{S}^{2 m-1}}$. Thanks to the explicit knowledge of the various terms composing $\mathcal{R}_{j}^{\text {out }}$ 's and $\mathcal{R}_{j}^{\text {in }}$ 's, in particular estimates (6.6) and (6.7), we can find $\kappa>0$ such that,

$$
\mathcal{S}: \mathcal{B}(\kappa, \delta)^{2} \rightarrow \mathcal{B}(\kappa, \delta)^{2}
$$

Now the conclusion follows immediately applying a Picard iteration scheme and standard regularity theory.

\section{Examples}

In this Section we list few examples where our results can be applied. We have confined ourselves to the case when $M$ is a toric Kähler-Einstein orbifold, but there is no doubt that this is far from a comprehensive list.

Theorem 7.1 Consider $\left(\mathbb{P}^{1} \times \mathbb{P}^{1}, \pi_{1}^{*} \omega_{F S}+\pi_{2}^{*} \omega_{F S}\right)$ and let $\mathbb{Z}_{2}$ act in the following way,

$$
\left(\left[x_{0}: x_{1}\right],\left[y_{0}: y_{1}\right]\right) \longrightarrow\left(\left[x_{0}:-x_{1}\right],\left[y_{0}:-y_{1}\right]\right) .
$$

It is immediate to check that this action is in $S U(2)$ with four fixed points,

$$
\begin{aligned}
& p_{1}=([1: 0],[1: 0]) \\
& p_{2}=([1: 0],[0: 1]) \\
& p_{3}=([0: 1],[1: 0]) \\
& p_{4}=([0: 1],[0: 1]) .
\end{aligned}
$$

The quotient space $X_{2}:=\mathbb{P}^{1} \times \mathbb{P}^{1} / \mathbb{Z}_{2}$ is a Kähler-Einstein, Fano orbifold and thanks to the embedding into $\mathbb{P}^{4}$

$$
\left(\left[x_{0}: x_{1}\right],\left[y_{0}: y_{1}\right]\right) \mapsto\left[x_{0}^{2} y_{0}^{2}: x_{0}^{2} y_{1}^{2}: x_{1}^{2} y_{0}^{2}: x_{1}^{2} y_{1}^{2}: x_{0} x_{1} y_{0} y_{1}\right],
$$

it is isomorphic to the intersection of singular quadrics

$$
\left\{z_{0} z_{3}-z_{4}^{2}=0\right\} \cap\left\{z_{1} z_{2}-z_{4}^{2}=0\right\}
$$

that by [2] is a limit of Kähler-Einstein surfaces, namely the intersection of two smooth quadrics. Since it is Kähler-Einstein, conditions for applying our construction become exactly the conditions of [5], so we have to verify that the matrix,

$$
\Theta\left(\mathbf{1}, s_{\omega} \mathbf{1}\right)=\left(\frac{s_{\omega}}{2} \varphi_{j}\left(p_{i}\right)\right)_{\substack{1 \leq i \leq 2 \\ 1 \leq j \leq 4}} .
$$

has full rank and there exist a positive element in $\operatorname{ker} \Theta\left(\mathbf{1}, s_{\omega} \mathbf{1}\right)$. It is immediate to see that we have,

$$
H^{0}\left(X_{2}, T^{(1,0)} X_{2}\right)=H^{0}\left(\mathbb{P}^{1} / \mathbb{Z}_{2}, T^{(1,0)}\left(\mathbb{P}^{1} / \mathbb{Z}_{2}\right)\right) \oplus H^{0}\left(\mathbb{P}^{1} / \mathbb{Z}_{2}, T^{(1,0)}\left(\mathbb{P}^{1} / \mathbb{Z}_{2}\right)\right) .
$$


Moreover,

$$
H^{0}\left(\mathbb{P}^{1} / \mathbb{Z}_{2}, T^{(1,0)}\left(\mathbb{P}^{1} / \mathbb{Z}_{2}\right)\right)
$$

is generated by holomorphic vector fields on $\mathbb{P}^{1}$ that vanish on points $[0: 1],[1: 0]$ so,

$$
\operatorname{dim}_{\mathbb{C}} H^{0}\left(\mathbb{P}^{1} / \mathbb{Z}_{2}, T^{(1,0)}\left(\mathbb{P}^{1} / \mathbb{Z}_{2}\right)\right)=1
$$

and an explicit generator is the vector field,

$$
V=z^{1} \partial_{1}
$$

We can compute explicitly its potential $\varphi_{V}$ with respect to $\omega_{F S}$ that is,

$$
\varphi_{V}\left(\left[z_{0}: z_{1}\right]\right)=-\frac{\left|z_{0} z_{1}\right|}{\left|z_{0}\right|^{2}+\left|z_{1}\right|^{2}}+\frac{1}{2}
$$

and it is easy to see that it is a well defined function and,

$$
\int_{\mathbb{P}^{1}} \varphi_{V} \omega_{F S}=0
$$

Summing up everything, we have that the matrix $\Theta\left(\mathbf{1}, s_{\omega} \mathbf{1}\right)$ for $X_{2}$ is a $2 \times 4$ matrix and can be written explicitly,

$$
\Theta\left(\mathbf{1}, s_{\omega} \mathbf{1}\right)=\frac{s_{\omega}}{2}\left(\begin{array}{cccc}
-1 & -1 & 1 & 1 \\
-1 & 1 & -1 & 1
\end{array}\right)
$$

that has rank 2 and every vector of type $(a, b, b, a)$ for $a, b>0$ lies in $\operatorname{ker} \Theta\left(\mathbf{1}, s_{\omega} \mathbf{1}\right)$.

Theorem 7.2 Consider $\left(\mathbb{P}^{2}, \omega_{F S}\right)$ and let $\mathbb{Z}_{3}$ act in the following way,

$$
\left[z_{0}: z_{1}: z_{2}\right] \longrightarrow\left[x_{0}: \zeta_{3} x_{1}: \zeta_{3}^{2} x_{2}\right] \quad \zeta_{3} \neq 1, \zeta_{3}^{3}=1 .
$$

It's immediate to check that this action is in $S U(2)$ with three fixed points,

$$
\begin{aligned}
& p_{1}=[1: 0: 0], \\
& p_{2}=[0: 1: 0], \\
& p_{3}=[0: 0: 1] .
\end{aligned}
$$

The quotient space $X_{3}:=\mathbb{P}^{2} / \mathbb{Z}_{3}$ is a Kähler-Einstein, Fano orbifold and it is isomorphic, via the embedding,

$$
\left[x_{0}: x_{1}: x_{2}\right] \mapsto\left[x_{0}^{3}: x_{1}^{3}: x_{2}^{3}: x_{0} x_{1} x_{2}\right],
$$

to the singular cubic surface in $\mathbb{P}^{3}$,

$$
\left\{z_{0} z_{1} z_{2}-z_{3}^{3}=0\right\}
$$

that by [27] we know to be a point of the boundary of the moduli space of Fano KählerEinstein surfaces, namely smooth cubic hypersurfaces. Again we have to verify that the matrix,

$$
\Theta\left(\mathbf{1}, s_{\omega} \mathbf{1}\right)=\left(\frac{2 s_{\omega}}{3} \varphi_{j}\left(p_{i}\right)\right)_{\substack{1 \leq i \leq 2 \\ 1 \leq j \leq 3}} .
$$


has full rank and there exist a positive element in $\operatorname{ker} \Theta\left(\mathbf{1}, s_{\omega} \mathbf{1}\right)$. It is immediate to see that we have,

$$
\operatorname{dim}_{\mathbb{C}} H^{0}\left(X_{3}, T^{(1,0)} X_{3}\right)=2,
$$

because $H^{0}\left(X_{3}, T^{(1,0)} X_{3}\right)$ it is generated by holomorphic vector fields on $\mathbb{P}^{2}$ vanishing at points $p_{1}, p_{2}, p_{3}$. Explicit generators are the vector fields,

$$
\begin{aligned}
& V_{1}=z^{1} \partial_{1}+z^{2} \partial_{2} \\
& V_{2}=z^{0} \partial_{0}+z^{1} \partial_{1} .
\end{aligned}
$$

We can compute explicitly their potentials $\phi_{V_{1}}, \phi_{V_{2}}$ with respect to $\omega_{F S}$ that are,

$$
\begin{aligned}
& \phi_{V_{1}}\left(\left[z_{0}: z_{1}: z_{2}\right]\right)=-\frac{\left|z^{0}\right|^{2}}{\left|z^{0}\right|^{2}+\left|z^{1}\right|^{2}+\left|z^{2}\right|^{2}}+\frac{1}{3} \\
& \phi_{V_{2}}\left(\left[z_{0}: z_{1}: z_{2}\right]\right)=-\frac{\left|z^{2}\right|^{2}}{\left|z^{0}\right|^{2}+\left|z^{1}\right|^{2}+\left|z^{2}\right|^{2}}+\frac{1}{3},
\end{aligned}
$$

and it is easy to see that are well defined functions and

$$
\int_{\mathbb{P}^{2}} \phi_{V_{1}} \frac{\omega_{F S}^{2}}{2}=\int_{\mathbb{P}^{2}} \phi_{V_{1}} \frac{\omega_{F S}^{2}}{2}=0,
$$

One can check that,

$$
\begin{aligned}
& \varphi_{1}=-3\left(\phi_{1}+2 \phi_{2}\right) \\
& \varphi_{2}=-3\left(2 \phi_{1}+\phi_{2}\right)
\end{aligned}
$$

is a basis of the space of potentials of holomorphic vector fields vanishing somewhere on $X_{3}$. Summing up everything, we have that the matrix $\Theta\left(\mathbf{1}, s_{\omega} \mathbf{1}\right)$ for $X_{3}$ is a $2 \times 3$ matrix and can be written explicitly,

$$
\Theta\left(\mathbf{1}, s_{\omega} \mathbf{1}\right)=\frac{2 s_{\omega}}{3}\left(\begin{array}{lll}
1 & -1 & 0 \\
0 & -1 & 1
\end{array}\right),
$$

that has rank 2 and every vector of type $(a, a, a)$ for $a>0$ lies in $\operatorname{ker} \Theta\left(\mathbf{1}, s_{\omega} \mathbf{1}\right)$.

Theorem 7.3 Let $X^{(1)}$ be the toric Kähler-Einstein threefold whose 1-dimensional fan $\Sigma_{1}^{(1)}$ is generated by points

$$
\Sigma_{1}^{(1)}=\{(1,3,-1),(-1,0,-1),(-1,-3,1),(-1,0,0),(1,0,0),(0,0,1),(0,0,-1),(1,0,1)\}
$$

and its 3-dimensional fan $\Sigma_{3}^{(1)}$ is generated by 12 cones

$$
\begin{aligned}
& C_{1}:=\langle(-1,0,-1),(-1,-3,1),(-1,0,0)\rangle \\
& C_{2}:=\langle(1,3,-1),(-1,0,-1),(-1,0,0)\rangle \\
& C_{3}:=\langle(-1,-3,1),(-1,0,0),(0,0,1)\rangle \\
& C_{4}:=\langle(1,3,-1),(-1,0,0),(0,0,1)\rangle \\
& C_{5}:=\langle(1,3,-1),(-1,0,-1),(0,0,-1)\rangle \\
& C_{6}:=\langle(-1,0,-1),(-1,-3,1),(0,0,-1)\rangle \\
& C_{7}:=\langle(-1,-3,1),(1,0,0),(0,0,-1)\rangle \\
& C_{8}:=\langle(1,3,-1),(1,0,0),(0,0,-1)\rangle
\end{aligned}
$$




$$
\begin{aligned}
C_{9} & :=\langle(1,3,-1),(0,0,1),(1,0,1)\rangle \\
C_{10} & :=\langle(-1,-3,1),(1,0,0),(1,0,1)\rangle \\
C_{11} & :=\langle(1,3,-1),(1,0,0),(1,0,1)\rangle \\
C_{12} & :=\langle(-1,-3,1),(0,0,1),(1,0,1)\rangle
\end{aligned}
$$

All these cones are singular and $C_{1}, C_{4}, C_{5}, C_{7}, C_{11}, C_{12}$ are cones relative to affine open subsets of $X^{(1)}$ containing a $S U(3)$ singularity, while the others are cones relative to affine open subsets of $X^{(1)}$ containing a $U(3)$ singularity.

The 3-anticanonical polytope $P_{-3 K_{X^{(1)}}}$ is the convex hull of vertices

$$
\begin{aligned}
P_{-3 K_{X^{(1)}}}:= & \langle(0,-2,-3),(-3,0,0),(-3,1,3),(0,0,3),(3,-2,0), \\
& (0,2,3),(0,0,-3),(-3,2,0),(-3,3,3),(3,0,0),(3,-1,-3),(3,-3,-3)\rangle
\end{aligned}
$$

With 2-faces

$$
\begin{aligned}
& F_{1}:=\langle(0,-2,-3),(3,-3,-3),(-3,0,0),(-3,1,3),(0,0,3),(3,-2,0)\rangle \\
& F_{2}:=\langle(-3,1,3),(0,0,3),(0,2,3),(-3,3,3)\rangle \\
& F_{3}:=\langle(0,0,3),(3,-2,0),(0,2,3),(3,0,0)\rangle \\
& F_{4}:=\langle(0,-2,-3),(-3,0,0),(0,0,-3),(-3,2,0)\rangle \\
& F_{5}:=\langle(3,-1,-3),(0,2,3),(0,0,-3),(-3,2,0),(-3,3,3),(3,0,0)\rangle \\
& F_{6}:=\langle(-3,0,0),(-3,1,3),(-3,2,0),(-3,3,3)\rangle \\
& F_{7}:=\langle(3,-1,-3),(0,-2,-3),(3,-3,-3),(0,0,-3)\rangle \\
& F_{8}:=\langle(3,-1,-3),(3,-3,-3),(3,-2,0),(3,0,0)\rangle
\end{aligned}
$$

We have the following correspondences between cones containing a $S U$ (3)-singularity and vertices of $P_{-3 K_{X^{(1)}}}$

$$
\begin{aligned}
C_{1} \longleftrightarrow F_{3} \cap F_{5} \cap F_{8} & =\{(3,0,0)\} \\
C_{4} \longleftrightarrow F_{1} \cap F_{7} \cap F_{8} & =\{(3,-3,-3)\} \\
C_{5} \longleftrightarrow F_{1} \cap F_{2} \cap F_{3} & =\{(0,0,3)\} \\
C_{7} \longleftrightarrow F_{2} \cap F_{5} \cap F_{7} & =\{(-3,3,3)\} \\
C_{11} \longleftrightarrow F_{1} \cap F_{4} \cap F_{6} & =\{(-3,0,0)\} \\
C_{12} \longleftrightarrow F_{4} \cap F_{5} \cap F_{7} & =\{(0,0,-3)\}
\end{aligned}
$$

Since in complex dimension 3 every $S U(3)$-singularity admits a Kähler crepant resolution it is then immediate to see that all assumptions of Theorem 1.1 are satisfied to get a partial resolution of this orbifold. We do not know whether we can also resolve the other $6 U(3)$ singularities since at the moment we do not know if a good local model for these groups exists.

Theorem 7.4 Let $X$ be the toric Kähler-Einstein threefold whose 1-dimensional fan $\Sigma_{1}$ is generated by points

$$
\Sigma_{1}=\{(0,3,1),(1,1,2),(1,0,0),(-1,0,0),(-2,-1,-2),(1,-3,-1)\}
$$

and its 3-dimensional fan $\Sigma_{3}$ is generated by 8 cones

$$
\begin{aligned}
& C_{1}:=\langle(0,3,1),(1,1,2),(-1,0,0)\rangle \\
& C_{2}:=\langle(0,3,1),(1,1,2),(1,0,0)\rangle
\end{aligned}
$$




$$
\begin{aligned}
& C_{3}:=\langle(0,3,1),(-1,0,0),(-2,-1,-2)\rangle \\
& C_{4}:=\langle(0,3,1),(1,0,0),(-2,-1,-2)\rangle \\
& C_{5}:=\langle(1,0,0),(-2,-1,-2),(1,-3,-1)\rangle \\
& C_{6}:=\langle(1,1,2),(-1,0,0),(1,-3,-1)\rangle \\
& C_{7}:=\langle(-1,0,0),(-2,-1,-2),(1,-3,-1)\rangle \\
& C_{8}:=\langle(1,1,2),(1,0,0),(1,-3,-1)\rangle
\end{aligned}
$$

The cones $C_{1}, C_{4}, C_{7}, C_{8}$ are relative to affine open subsets of $X$ containing a $S U$ (3) singularity and the other cones are relative to affine open subsets of $X$ containing a $U(3)$ singularity. The 5-anticanonical polytope $P_{-5 K_{X}}$ is the convex hull of vertices

$$
\begin{aligned}
P_{-5 K_{X}}:= & \langle(5,-1,-2),(5,0,-5),(-5,-2,1),(-5,0,0), \\
& (5,5,-5),(-5,-5,10),(-5,-3,9),(5,6,-8)\rangle
\end{aligned}
$$

With 2-faces

$$
\begin{aligned}
& F_{1}:=\langle(5,0,-5),(-5,-2,1),(-5,0,0),(5,6,-8)\rangle \\
& F_{2}:=\langle(5,-1,-2),(5,0,-5),(-5,-2,1),(-5,-5,10)\rangle \\
& F_{3}:=\langle(5,-1,-2),(5,0,-5),(5,5,-5),(5,6,-8)\rangle \\
& F_{4}:=\langle(5,-1,-2),(5,5,-5),(-5,-5,10),(-5,-3,9)\rangle \\
& F_{5}:=\langle(-5,-2,1),(-5,0,0),(-5,-5,10),(-5,-3,9)\rangle \\
& F_{6}:=\langle(-5,0,0),(5,5,-5),(-5,-3,9),(5,6,-8)\rangle
\end{aligned}
$$

We have the following correspondences between cones containing a $S U$ (3)-singularity and vertices of $P_{-5 K_{X}}$

$$
\begin{aligned}
& C_{1} \longleftrightarrow F_{1} \cap F_{2} \cap F_{5}=\{(-5,-2,1)\} \\
& C_{4} \longleftrightarrow F_{2} \cap F_{3} \cap F_{4}=\{(5,-1,-2)\} \\
& C_{7} \longleftrightarrow F_{4} \cap F_{5} \cap F_{6}=\{(-5,-3,9)\} \\
& C_{8} \longleftrightarrow F_{1} \cap F_{3} \cap F_{6}=\{(5,6,-8)\}
\end{aligned}
$$

Since in complex dimension 3 every $S U$ (3)-singularity admits a Kähler crepant resolution it is then immediate to see that all assumptions of Theorem 1.1 are satisfied to get a partial resolution of this orbifold. Whether we can also resolve the other $4 U(3)$ singularities we do not know since at the moment we do not know if a good local model for these groups exists.

Theorem 7.5 Let $Y$ be the toric Kähler-Einstein threefold whose 1-dimensional fan $\Sigma_{1}$ is generated by points

$$
\Sigma_{1}=\{(2,-1,0),(1,3,1),(0,0,1),(-3,-2,-2)\}
$$

and its 3-dimensional fan $\Sigma_{3}$ is generated by 6 cones

$$
\begin{aligned}
& C_{1}:=\langle(1,3,1),(0,0,1),(-3,-2,-2),\rangle \\
& C_{2}:=\langle(2,-1,0),(0,0,1),(-3,-2,-2)\rangle \\
& C_{3}:=\langle(2,-1,0),(1,3,1),(-3,-2,-2)\rangle \\
& C_{4}:=\langle(2,-1,0),(1,3,1),(0,0,1)\rangle
\end{aligned}
$$

The cone $C_{1}$ is relative to affine open subsets of $Y$ containing a $S U(3)$ singularity and the other cones are relative to affine open subsets of $Y$ containing a $U(3)$ singularity. 
The 7-anticanonical polytope $P_{-7 K_{X^{(6)}}}$ is the convex hull of vertices

$$
P_{-7 K_{X}^{(6)}}:=\langle(1,9,-7),(-3,1,-7),(9,-3,-7),(-7,-7,21)\rangle
$$

With 2-faces

$$
\begin{aligned}
& F_{1}:=\langle(-3,1,-7),(9,-3,-7),(-7,-7,21)\rangle \\
& F_{2}:=\langle(1,9,-7),(9,-3,-7),(-7,-7,21)\rangle \\
& F_{3}:=\langle(1,9,-7),(-3,1,-7),(-7,-7,21)\rangle \\
& F_{4}:=\langle(1,9,-7),(-3,1,-7),(9,-3,-7)\rangle
\end{aligned}
$$

We have the following correspondences between cones containing a $S U$ (3)-singularity and vertices of $P_{-7 K_{X^{(6)}}}$

$$
C_{1} \longleftrightarrow F_{1} \cap F_{2} \cap F_{4}=\{(9,-3,-7)\}
$$

It is now clear that this example does not satisfy either the balancing condition on the $S U$ (3) point, nor the one found in [3] on the remaining $3 U(3)$ singularities. Ito would be very interesting to know whether this examples can be actually desingularized by Kcsc manifolds.

Acknowledgements We wish to express our deep gratitude to Gavin Brown and Alexander Kasprzyk for their help in not drowning in the Fano toric threefolds world. The authors have been partially supported by the FIRB Project "Geometria Differenziale Complessa e Dinamica Olomorfa".

\section{References}

1. Arezzo, C., Della Vedova, A., Mazzieri, L.: K-stability, Futaki invariants and cscK metrics on orbifold resolutions (2017). arXiv:1808.08420

2. Arezzo, C., Ghigi, A., Pirola, G.P.: Symmetries, quotients and Kähler-Einstein metrics. J. Reine Angew. Math. 591, 177-200 (2006)

3. Arezzo, C., Lena, R., Mazzieri, L.: On the resolution of extremal and constant scalar curvature Kähler orbifolds. Int. Math. Res. Not. 21, 6415-6452 (2016)

4. Arezzo, C., Pacard, F.: Blowing up and desingularizing constant scalar curvature Kähler manifolds. Acta Math. 196(2), 179-228 (2006)

5. Arezzo, C., Pacard, F.: Blowing up Kähler manifolds with constant scalar curvature. II. Ann. Math. (2) 170(2), 685-738 (2009)

6. Arezzo, C., Pacard, F., Singer, M.: Extremal metrics on blowups. Duke Math. J. 157(1), 1-51 (2011)

7. Calabi, E.: Extremal Khler Metrics. II. Differential Geometry and Complex Analysis, pp. 95-114. Springer, Berlin (1985)

8. Conlon, R.J., Hein, H.-J.: Asymptotically conical Calabi-Yau manifolds. I'. Duke Math. J. 162(15), 2855-2902 (2013)

9. Brown, G., Kasprzyk, A.: http://www.grdb.co.uk (2015)

10. Brown, G., Kasprzyk, A.: Graded Ring Database (2013). http://grdb.lboro.ac.uk/

11. Calderbank, D.M.J., Singer, M.: Einstein metrics and complex singularities. Invent. Math. 156(2), 405443 (2004)

12. Goto, R.: Calabi-Yau structures and Einstein-Sasakian structures on crepant resolutions of isolated singularities. J. Math. Soc. Jpn. 64, 1005-1052 (2012)

13. Hein, H.-J., LeBrun, C.: Mass in Kähler geometry. Commun. Math. Phys. 347(1), 183-221 (2016)

14. Honda, N.: Deformation of LeBrun's ALE metrics with negative mass. Commun. Math. Phys. 322(1), 127-148 (2013)

15. Joyce, D.: Compact Manifolds with Special Holonomy. Oxford Mathematical Monographs. Oxford University Press, Oxford (2000)

16. Kasprzyk, A.: Canonical toric Fano threefolds. Can. J. Math. 62(6), 1293-1309 (2010)

17. LeBrun, C., Singer, M.: A Kummer-type construction of self-dual 4-manifolds. Math. Ann. 300, 165-180 (1994) 
18. Mazzieri, L.: Generalized connected sum construction for nonzero constant scalar curvature metrics. Commun. Partial Differ. Equ. 33, 1-17 (2008)

19. Mazzieri, L.: Generalized connected sum construction for scalar flat metrics. Manuscr. Math. 129, 137168 (2009)

20. Mazzieri, L.: Generalized gluing for Einstein constraint equations. Calc. Var. Partial Differ. Equ. 34, 453-473 (2009)

21. Odaka, Y., Spotti, C., Sun, S.: Compact Moduli Spaces of Del Pezzo Surfaces and Kähler-Einstein Metrics (2015). arXiv:1210.0858 (to appear in J. Diff. Geom.)

22. Page, D.N.: A physical picture of the $K 3$ gravitational instanton. Phys. Lett. 80, 55-57 (1978)

23. Pacard, F.: Lectures on connected sum constructions in geometry and nonlinear analysis. http://www. cmls.polytechnique.fr/perso/pacard.frank/Publications/Lecture-Part-I.pdf (2008)

24. Rollin, Y., Singer, M.: Non-minimal scalar-flat Kähler surfaces and parabolic stability. Invent. Math. 162(2), 235-270 (2005)

25. Székelyhidi, G.: Blowing up extremal Kähler manifolds II. Invent. Math. 200(3), 925-977 (2015)

26. Székelyhidi, G.: On blowing up extremal Kähler manifolds. Duke Math. J. 161(8), 1411-1453 (2012)

27. Tian, G.: On Calabi's conjecture for complex surfaces with positive first Chern class. Invent. Math. 101, 101-172 (1990)

28. Topiwala, P.: A new proof of the existence of Kähler-Einstein metrics on K3. I, II. Invent. Math. 89, 425-448 (1987) (449-454)

29. van Coevering, C.: Ricci-flat Kähler metrics on crepant resolutions of Kähler cones. Math. Ann. 347(3), 581-611 (2010) 